(c) 2005 International Press

Adv. Theor. Math. Phys. 9 (2005) 31-128

\title{
Large $N$ Duality for Compact Calabi-Yau Threefolds
}

\author{
Duiliu-Emanuel Diaconescu, Bogdan Florea
}

\author{
Department of Physics and Astronomy \\ Rutgers University \\ Piscataway, NJ 08855-0849, USA
}

\begin{abstract}
We study geometric transitions for topological strings on compact Calabi-Yau hypersurfaces in toric varieties. Large $N$ duality predicts an equivalence between topological open and closed string theories connected by an extremal transition. We develop new open string enumerative techniques and perform a high-precision genus zero test of this conjecture for a certain class of toric extremal transitions. Our approach is based on (a) an open string version of Gromov-Witten theory with convex obstruction bundle and (b) an extension of Chern-Simons theory treating the framing as a formal variable.
\end{abstract}

\section{Introduction}

Large $N$ duality $[21,23,53]$ predicts a highly nontrivial relation between open and closed topological strings on Calabi-Yau three-folds connected by an extremal transition. Originally formulated for local conifold transitions, the duality has been extended in various directions in refs. [2-5,

e-print archive: http://lanl.arXiv.org/abs/hep-th/0302076 
15-18, 29, 30, 38-40, 47, 52, 54]. The generalizations considered in refs. [4, 5, $15,16]$ are especially interesting since they shed a new light on the structure of Gromov-Witten invariants of toric Calabi-Yau manifolds. These developments are based on an earlier idea of Witten [58], who outlines a beautiful approach to A-model topological open string amplitudes. The main idea of this approach is based on a subtle combination of Chern-Simons theory and open string enumerative geometry, which will be discussed in detail below. At present, open string enumerative geometry is not a fully developed mathematical formalism. The basic principles understood so far have been developed in refs. $[25,33,45]$ in the context of toric Calabi-Yau threefolds. Additional work on the subject exhibiting various points of view can be found in refs. $[1,2,15,16,24,31,32,41-43,48,49]$. In particular, the work of Diaconescu et al. $[15,16]$ was successfully applied to geometric transitions for noncompact Calabi-Yau hypersurfaces in toric varieties.

In this paper, we consider large $N$ duality for extremal transitions between compact Calabi-Yau hypersurfaces in toric varieties. Geometric transitions for compact Calabi-Yau manifolds have been proposed in ref. [57], but no concrete results are known so far. There are many conceptual as well as technical issues that have to be addressed in this context. Perhaps the most challenging obstacle is the absence of a rigorous mathematical formalism for open string amplitudes on compact Calabi-Yau three-folds.

Keeping the details to a minimum, let us summarize our results. We develop a computational approach to genus zero open string A-model amplitudes on a certain class of compact Calabi-Yau target manifolds. This approach follows the basic principles outlined in ref. [58] supplemented by a heavy use of equivariant enumerative techniques. There are two key aspects in the whole process. First, we develop an open string version of the convex obstruction bundle approach usually encountered in Gromov-Witten theory. Second, we propose a formal extension of standard Chern-Simons theory in which the framing is regarded as a formal variable. In particular, it could take fractional values. Using these two elements, plus a great deal of patience, we run a successful genus zero test of large $N$ duality for compact Calabi-Yau hypersurfaces in toric varieties. Along the way, we gain new insights into the structure of Gromov-Witten invariants, and we also clarify some aspects of local geometric transitions $[5,15,16]$. A more detailed overview of the general set-up and the results is included in the next section.

The paper is structured as follows. In Section 2, we present the stage, the cast of characters and outline the main plot. Section 3 consists of a conceptual discussion of open string enumerative geometry for compact CalabiYau manifolds. In Section 4, we describe the main examples to be used 
for concrete computations. We revisit local geometric transitions in Section 5 , focusing on the interplay between equivariant enumerative geometry and Chern-Simons theory. The main outcome of this discussion is a notion of formal Chern-Simons expansion which will prove crucial for compact threefolds. Sections 6 and 7 are devoted to an implementation of our program in a concrete model.

\section{Large $N$ duality for compact three-folds — preliminary remarks}

Although the problem can be formulated in a more general context, in this paper we will consider only toric extremal transitions between Calabi-Yau hypersurfaces $[6,8,50]$. Recall [7] that a family of Calabi-Yau hypersurfaces $Y$ in a toric variety $\mathcal{Z}$ is described by a reflexive Newton polyhedron $\mathbb{P}_{\Delta_{Y}}$. The class of extremal transitions we are interested in is described by an embedding of reflexive polyhedra $\mathbb{P}_{\Delta_{\tilde{Y}}} \subset \mathbb{P}_{\Delta_{Y}}$. It has been shown in refs. [6, $8,50]$ that such an embedding gives rise to a commutative diagram of the form

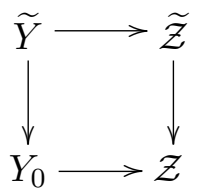

The vertical arrows are extremal contractions and $Y_{0}$ is a singular hypersurface in the family $Y$ corresponding to special values of complex structure parameters. The toric extremal contraction $\widetilde{\mathcal{Z}} \longrightarrow \mathcal{Z}$ admits a simple toric description in terms of a similar embedding of dual reflexive polyhedra $\mathbb{P}_{\nabla_{Y}} \subset \mathbb{P}_{\nabla_{\tilde{Y}}}$. The vertices of $\mathbb{P}_{\nabla_{\widetilde{Y}}}$ not belonging to $\mathbb{P}_{\nabla_{Y}}$ correspond to toric divisors on $\widetilde{\mathcal{Z}}$ which are contracted in the process. In the following, we will consider only singular hypersurfaces $Y_{0}$ with isolated ordinary double points. Moreover, the contraction $\widetilde{Y} \longrightarrow Y$ is a simultaneous crepant resolution of the nodes. Also, we will assume $\mathcal{Z}, \widetilde{Z}$ to be smooth toric four-folds. This can always be achieved by triangulating the toric fans.

To be more concrete, let us consider a one-parameter family $Y_{\mu}$ of hypersurfaces in $\mathcal{Z}$ which degenerates to $Y_{0}$ for $\mu=0$. We denote by $v$ the number of nodal singularities of $Y_{0}$ and by $\left[L_{1}\right], \ldots,\left[L_{v}\right]$ the corresponding homology classes of vanishing cycles on $Y_{\mu}$. The $\left[L_{i}\right], i=1, \ldots, v$, generate a rank $(v-r)$ sublattice $H_{v} \subset H_{3}\left(Y_{\mu}, \mathbb{Z}\right)$, where $r$ is the number of relations among vanishing cycles. Without loss of generality, we can assume that 
$\left[L_{r+1}\right], \ldots,\left[L_{v}\right]$ is an integral system of generators for $H_{v}$. Therefore, we have the following linear relations with integral coefficients

$$
\left[L_{i}\right]=\sum_{m=r+1}^{v} a_{i, m}\left[L_{m}\right]
$$

for $i=1, \ldots, r$.

The exceptional locus of the crepant resolution $\tilde{Y} \longrightarrow Y$ consists of $v$ isolated $(-1,-1)$ curves $C_{1}, \ldots, C_{v}$ on $\tilde{Y}$. Clemens [12] shows that the curve classes $\left[C_{1}\right], \ldots,\left[C_{v}\right] \in H_{2}(\widetilde{Y}, \mathbb{Z})$ are subject to $(v-r)$ relations, so that we are left with $r$ independent curve classes on $\widetilde{Y}$. Without loss of generality, we can take them to be $\left[C_{1}\right], \ldots,\left[C_{r}\right]$. The numbers $(v, r)$ can be related to the toric data described in the previous paragraph using Batyrev's formulae [7]. We will work under the simplifying assumption that all extra $r(1,1)$ classes on $\tilde{Y}$ are toric, i.e., $r=h^{1,1}(\widetilde{\mathcal{Z}})-h^{1,1}(\mathcal{Z})$. Therefore, the curves $C_{1}, \ldots, C_{r}$ determine $r$ independent curve classes on $\widetilde{\mathcal{Z}}$ as well. We will understand why this assumption is necessary by the end of this section.

In this geometric context, we consider an open string A-model specified by wrapping $N_{i}$ topological branes on a collection of vanishing cycles $L_{i} \in\left[L_{i}\right]$ [58]. In order to obtain a well-defined topological open string A-model, the cycles $L_{i}$ should be Lagrangian. Physical considerations $[26,55]$ suggest that each class $\left[L_{i}\right]$ should contain a unique special Lagrangian cycle homeomorphic to $S^{3}$ in order for the transition to make sense in string theory. Unfortunately, this does not seem to be a well-established mathematical result for a generic deformation $Y_{\mu}$. For the remaining part of this section, we will assume this to be true. In later sections, we will work with a special degeneration of $Y_{\mu}$ where one can explicitly construct such Lagrangian representatives.

In a topological theory, there should not be any constraint on the $N_{i}$ arising from flux conservation. If we regard our model as the topological sector of an underlying superstring theory, the total D-brane charge has to be zero because $\widetilde{Y}$ is compact. Therefore, we must have

$$
\sum_{i=1}^{v} N_{i}\left[L_{i}\right]=0 .
$$

Although this is not strictly a necessary condition in the topological theory, it seems to be required in order to match the number of parameters in the context of large $N$ duality. This can be seen by substituting equation (2.2) in equation $(2.3)$ and using the fact that $\left[L_{r+1}\right], \ldots,\left[L_{v}\right]$ form a basis. We 
obtain

$$
N_{m}+\sum_{i=1}^{r} N_{i} a_{i, m}=0, \quad m \geq r+1 .
$$

This shows that there are only $r$ independent D-brane charges $N_{1}, \ldots, N_{r}$ in one-to-one correspondence with the exceptional curves $C_{1}, \ldots, C_{r}$.

As topological A-model amplitudes depend on Kähler moduli, we choose specific parameterizations of the Kähler cones of $\mathcal{Z}, \widetilde{\mathcal{Z}}$ as follows

$$
J=\sum_{\alpha=1}^{h^{1,1}(\mathcal{Z})} t_{\alpha} J_{\alpha}, \quad \widetilde{J}=\sum_{\gamma=1}^{h^{1,1}(\widetilde{\mathcal{Z}})} \widetilde{t}_{\gamma} \widetilde{J}_{\gamma} .
$$

Here $J_{\alpha}, \alpha=1, \ldots, h^{1,1}(\mathcal{Z})$, and, respectively, $\widetilde{J}_{\gamma}, \gamma=1, \ldots, h^{1,1}(\widetilde{\mathcal{Z}})$, are Kähler cone generators. Note that $t_{\alpha}$ and $\widetilde{t}_{\gamma}$ are classical Kähler parameters. The topological amplitudes should be written in terms of flat Kähler parameters, which include instanton corrections. Abusing notation, we will use the same symbols for classical and flat Kähler parameters. The distinction should be clear from the context.

After all these preliminary remarks, we are ready to discuss the large $N$ duality conjecture in the present context. Following the general philosophy of [22], large $N$ duality should predict a relation between topological closed strings on $\widetilde{Y}$ and topological open strings on $(Y, L)$, where $L=\cup_{i=1}^{v} L_{i}$. Therefore, it would be tempting to conjecture a direct relation between the Gromov-Witten expansion of $\widetilde{Y}$ and a hypothetical open string expansion associated to $(Y, L)$. While such a relation may exist abstractly, at present such a direct approach is out of reach. Instead, we will concentrate only on the genus zero part of the duality and follow one of the main lessons of mirror symmetry for Calabi-Yau hypersurfaces. In the linear sigma model approach, the genus zero Gromov-Witten expansion of a hypersurface $\widetilde{Y}$ is defined extrinsically in terms of maps to the ambient toric variety $\widetilde{\mathcal{Z}}$, using the convex obstruction bundle approach. Roughly, this means that one counts holomorphic maps to $\widetilde{\mathcal{Z}}$, subject to an algebraic constraint. This produces a genus zero closed string expansion of the form

$$
\mathcal{F}_{\widetilde{Y} ; \mathrm{cl}}^{(0)}\left(g_{\mathrm{s}}, \widetilde{t}_{\gamma}\right)=g_{\mathrm{s}}^{-2} \sum_{\widetilde{\beta} \in H_{2}(\widetilde{\mathcal{Z}, \mathbb{Z})}} \widetilde{C}_{0, \widetilde{\beta}} \mathrm{e}^{-\langle\widetilde{J}, \widetilde{\beta}\rangle},
$$

where the coefficients $\widetilde{C}_{0, \widetilde{\beta}}$ have a standard definition in terms of intersection theory on the moduli space of stable maps to $\widetilde{\mathcal{Z}}$ which will be reviewed in the next section. It is worth noting here that only the data of $\widetilde{\mathcal{Z}}$ and the linear system $|\widetilde{Y}|$ enter the definition of $\widetilde{C}_{0, \widetilde{\beta}}$. Therefore, these coefficients are manifestly independent of complex structure deformations of $Y$. 
Following the same idea, one would like to define a similar instanton sum for topological open strings on $(Y, L)$ using maps to the ambient space $\mathcal{Z}$ with boundary conditions on $L$. This is a very delicate construction for many reasons explained in detail in Section 3. One of the obvious problems is that such a construction would not be manifestly independent on complex structure deformations of $Y$, since the cycles $L$ move with $Y$. Moreover, for fixed $(Y, L)$, there is no rigorous mathematical formalism for counting open string maps in an appropriate sense. Finally, in open string theories one has to sum over highly degenerate maps which contract a bordered Riemann surface into an infinitely thin ribbon graph [58]. From a physical point of view, this yields a Chern-Simons sector of the topological string theory consisting of $v$ Chern-Simons theories with gauge groups $U\left(N_{i}\right)$ supported on the cycles $L_{i}, i=1, \ldots, v$ [58]. The coupling of this sector to open string instantons is a very subtle issue not fully understood at this stage. This subject will be discussed in detail in Section 5 for local models and Section 7 for compact Calabi-Yau three-folds.

We will not attempt to fill all the gaps mentioned in the previous paragraph in the present paper. Instead, we will assume invariance under complex structure deformations, and construct a genus zero open string generating functional using a special degeneration $(\bar{Y}, \bar{L})$ of the pair $(Y, L)$. The construction relies heavily on the existence of a toric action on $\mathcal{Z}$ which induces a subtorus action on $\bar{Y}$. The existence of a suitable degeneration places important restrictions on the range of validity of this approach. As explained in Sections 3 and 6, such a direct construction can be carried out only for extremal transitions in which the nodal points are fixed points of the torus action on $\mathcal{Z}$. Without giving more details here, let us note that in favorable cases we can construct a genus zero open string generating functional of the form ${ }^{1}$

$$
\mathcal{F}_{(Y, L) ; \mathrm{op}}^{(0)}\left(g_{\mathrm{s}}, t_{\alpha}, \lambda_{i}\right)=\mathcal{F}_{Y ; \mathrm{cl}}^{(0)}\left(g_{\mathrm{s}}, t_{\alpha}\right)+\sum_{\beta \in H_{2}(\mathcal{Z}, \bar{L} ; \mathbb{Z})} F_{\beta}^{(0)}\left(g_{\mathrm{s}}, \lambda_{i}\right) \mathrm{e}^{-\langle J, \beta\rangle}
$$

Here $\lambda_{i}=g_{\mathrm{s}} N_{i}$ are the 't Hooft coupling constants for the $v$ Chern-Simons theories on $L_{i}$. Given the relations (2.4), we have only $r$ independent 't Hooft couplings $\lambda_{i}, i=1, \ldots, r$, in formula (2.7). This matches the number of exceptional curve classes on $\widetilde{Z}$, according to the paragraph below equation (2.2). In the right-hand side of $(2.7), \mathcal{F}_{Y ; \mathrm{cl}}\left(g_{\mathrm{s}}, t_{\alpha}\right)^{(0)}$ is the genus zero closed

\footnotetext{
${ }^{1}$ Here we ignore some subtleties related to open string quantum corrections to the flat Kähler parameters. Since this is a general discussion, these corrections can be absorbed in the definition of $F_{\beta}^{(0)}\left(g_{\mathrm{s}}, \lambda_{i}\right)$.
} 
string Gromov-Witten expansion of $Y$

$$
\mathcal{F}_{Y ; \mathrm{cl}}^{(0)}\left(g_{\mathrm{s}}, t_{\alpha}\right)=g_{\mathrm{s}}^{-2} \sum_{\beta \in H_{2}(\mathcal{Z}, \mathbb{Z})} C_{0, \beta} \mathrm{e}^{-\langle J, \beta\rangle} .
$$

The construction of the generating functional in equation (2.7) is discussed from a conceptual point of view in Section 3, and carried out for a concrete compact example in Sections 6 and 7.

Granting the existence of a well-defined open string partition function, what are the predictions of large $N$ duality? The conjecture is that the two generating functionals (2.6) and (2.7) should be equal, subject to a certain identification of parameters which is the duality map. To this end, let us introduce a different system of generators $\left(\pi^{*}\left(J_{\alpha}\right), D_{i}\right), \alpha=1, \ldots, h^{1,1}(\mathcal{Z})$, $i=1, \ldots, r$, of $H^{1,1}(\widetilde{\mathcal{Z}})$. Here $\pi: \widetilde{\mathcal{Z}} \longrightarrow \mathcal{Z}$ is the contraction map and $D_{i}, i=$ $1, \ldots, r$, are divisor classes on $\widetilde{\mathcal{Z}}$ such that $\pi_{*}\left(D_{i}\right)=0$ and $D_{i} \cdot C_{j}=\delta_{i j}$ for $1 \leq i, j \leq r$. Then, modulo some subtleties concerning open string Kähler moduli which will be considered later, the duality map is specified by the following relations

$$
\widetilde{J}=\pi^{*} J-i \sum_{i=1}^{r} \lambda_{i} D_{i} .
$$

Using this map, we will test the duality predictions for a concrete example in Section 7.

\section{Open string A-model approach - general considerations}

In principle, large $N$ duality should hold for any conifold transition between Calabi-Yau three-folds. However, our current understanding of topological open string A-model amplitudes is restricted to the so-called local models, i.e., noncompact Calabi-Yau three-folds admitting a torus action. The case of compact Calabi-Yau three-folds is especially hard, no concrete results being known so far. In this paper, we would like to propose an approach to this problem for a certain class of hypersurfaces in toric varieties. This section is a rather general exposition of the basic principles. The details will be worked out for concrete examples in the next sections.

Let us start the discussion with some general considerations on open string A-models, following ref. [58]. Suppose we have a model defined by $v$ Lagrangian cycles $L_{1}, \ldots, L_{v}$ as in the previous section. The target space effective action of such a theory consists of $v$ Chern-Simons theories with gauge groups $U\left(N_{i}\right), i=1, \ldots, v$, supported on the $v$ Lagrangian cycles. This is a universal sector of the theory which is present for any target 
manifold. Then one has instanton corrections to the Chern-Simons theory obtained by summing over holomorphic maps $f: \Sigma_{g, h} \longrightarrow Y$ with Lagrangian boundary conditions on the $L_{i}$. Obviously, these corrections depend on the geometry of the target manifold, and the sum should be properly formulated in terms of intersection theory on a moduli space of stable maps $\bar{M}_{g, h}(Y, L)$. There is, however, an important subtlety in this approach which may be easier to understand for a concrete example. Suppose one can find a rigid holomorphic disc $D_{i}$ embedded in $Y$ with boundary $\Gamma_{i} \subset L_{i}$. Let $\beta=\left[D_{i}\right] \in H_{2}(Y, L ; \mathbb{Z})$ and let $\gamma=\left[\Gamma_{i}\right] \in H_{1}\left(L_{i}, \mathbb{Z}\right)$. This yields an instanton correction of the form

$$
\mathrm{e}^{-\tau_{i}} \operatorname{Tr}\left(\mathrm{P} \exp \int_{\Gamma_{i}} f^{*} A_{i}\right)
$$

to the $U\left(N_{i}\right)$ Chern-Simons theory on $L_{i}$. Here $\tau_{i}$ is the symplectic area of the disc and $A_{i}$ is the $U\left(N_{i}\right)$ gauge field on $L_{i}$. It is important to note that $A_{i}$ is not a fixed flat connection on $L_{i}$. Since the cycle is compact, $A_{i}$ is a dynamical variable, and one should integrate over all connections. However, this proposal immediately raises a puzzle if the disc $D_{i}$ moves in a family on $Y$. Since $A_{i}$ is not flat, the holonomy factor $\operatorname{Tr}\left(\mathrm{P} \exp \int_{\Gamma_{i}} f^{*} A_{i}\right)$ depends on the position of $\Gamma$ in $L_{i}$. Therefore, it is not clear how to write down the instanton corrections in this case. Since A-models make sense on general symplectic manifolds equipped with a compatible almost complex structure, one solution is to deform to a generic situation in which we have a finite number of rigid discs. Then each disc would yield a correction of the form (3.1). Conceptually, this is a satisfying solution, but it is not very effective in practice if one is interested in explicit numerical computations. The solution proposed in refs. $[15,16]$ is based on localization with respect to a toric action. More precisely, if $Y$ admits a torus action which preserves the Lagrangian cycles, the idea is to sum only over fixed points of the induced action on the moduli space of maps. To each fixed point, one can associate an instanton expansion consisting of terms of the form (3.1) and multicovers. In order to obtain the topological free energy, one has to first sum the instanton expansions over all fixed points and then perform the Chern-Simons functional integral with all these corrections taken into account. Such computations have been performed for noncompact manifolds in refs. $[5,15,16]$.

At this point, it may be helpful to emphasize the distinction between compact and noncompact cycles. If $L_{i}$ were a noncompact cycle in some noncompact Calabi-Yau manifold, one could take $A_{i}$ to be a fixed flat connection on $L_{i}$. For a flat connection, the holonomy factor $\operatorname{Tr}\left(\mathrm{P} \exp \int_{\Gamma_{i}} f^{*} A_{i}\right)$ 
depends only on the homology class $\gamma$. Therefore, for fixed homology classes $(\beta, \gamma)$ the instanton series can be thought of a formal series in two sets of variables [33]. The computation of topological amplitudes is then reduced to the construction of a virtual cycle of dimension zero on the moduli space of stable open string maps. The structure of this moduli space is not very well understood at the moment, hence there are various technical problems with such a construction. Nevertheless, one can go a long way exploiting the presence of a torus action $[25,33,45,49]$. Even if the moduli space is very complicated, the fixed loci of the induced torus action are much simpler and can be described in detail. Then one can define the virtual cycle by directly summing over invariant maps. Since the moduli space has boundary, the resulting invariants will depend on a choice of boundary conditions, which can be encoded in a choice of the torus weights [33]. Therefore, we obtain a family of invariants depending on a discrete set of choices.

If the cycles $L_{i}$ are compact, the local contribution of a fixed locus is to be interpreted as a series of corrections to Chern-Simons theory, as explained above. As a result, each term in this series will be a rational function of the weights of the torus action. The final expression for the open string free energy is obtained by computing the free energy of the corrected ChernSimons action. At this point, we seem to obtain a puzzle since a priori the resulting open string amplitudes will depend on the weights of the torus action. This dependence is not physically acceptable in the context of large $N$ duality, since there are no discrete ambiguities in the dual closed string expansion.

The resolution of this puzzle is that the choice of the toric weights has to be correlated to the framing of the knots $\Gamma_{i}$ in Chern-Simons theory in such a way that the final result agrees with the closed string dual. Although this idea has been concretely applied in certain local models in refs. $[15,16]$, the general rules behind the weight-framing correspondence are not well understood at present. In particular, integrality of the framing imposes very strong constraints on the allowed weights, which may not even have solution in many cases. One of the main results of this work is a general rule for this correspondence based on a certain extension of the Chern-Simons expansion treating the framing as a formal variable. This will be discussed at length for local models in Section 5, and for compact Calabi-Yau three-folds in Section 7 .

For the remaining part of this section, we will focus on localization questions for open string maps to compact Calabi-Yau hypersurfaces. Our goal is to develop an algorithm for the computation of the local contributions associated with fixed loci in such situations. 


\subsection{Open string morphisms - the convex obstruction bundle approach}

Open string A-model localization techniques have been first developed in refs. [25, 33, 45] for noncompact Lagrangian cycles in noncompact toric threefolds. An extension of these techniques to compact Lagrangian cycles has been discussed in refs. $[15,16]$. In the later cases, the ambient Calabi-Yau three-fold was a noncompact hypersurface in a toric variety. The invariants computed there may be regarded as an open string version of closed string local Gromov-Witten invariants [13].

Briefly, the typical situation encountered in closed string computations is the following. One has a toric Calabi-Yau variety $X$ isomorphic to the total space of a holomorphic vector bundle $N$ over a base $B$. Usually $B$ is a rational curve or a toric Fano surface, so that the zero section of the fibration $N \longrightarrow B$ is rigid in $X$. The basic data of the enumerative problem is given by the negative obstruction bundle $\mathcal{E}=R^{1} \rho_{*}\left(e v^{*} N\right)$ on the moduli space of stable maps $\bar{M}_{g, 0}(B, \beta)$, with fixed class $\beta \in H_{2}(B, \mathbb{Z})$. Here $e v: \bar{M}_{g, 1}(B, \beta) \longrightarrow B$ is the evaluation map and $\rho: \bar{M}_{g, 1}(B, \beta) \longrightarrow \bar{M}_{g, 0}(B, \beta)$ is the forgetful map. This is standard material. The local Gromov-Witten invariants are defined by integrating the Euler class of $\mathcal{E}$ against the virtual fundamental class $\left[\bar{M}_{g, 0}(B, \beta)\right]$. Alternatively, we can define the same invariants as the degree of the virtual cycle $\left[\bar{M}_{g, 0}(X, \beta)\right]$, which is of dimension zero. One can show without too much effort that the two definitions are equivalent (see, e.g., Section 9.2.2 of ref. [12]). Since $B$ is toric, we can in fact explicitly evaluate these numbers using localization techniques $[20,36]$.

By contrast, compact Calabi-Yau three-folds, such as hypersurfaces in toric varieties, require a different approach. The obvious difficulty in applying localization techniques to this case is the absence of a torus action on a smooth generic hypersurface. For genus zero maps, this problem is elegantly solved by the convex obstruction bundle approach. Shifting perspective, the genus zero numerical invariants are formulated in terms of the moduli space $\bar{M}_{0,0}(\mathcal{Z}, \beta)$ of stable maps to the ambient toric variety $\mathcal{Z}$, which admits a toric action. Here $\beta \in H_{2}(\mathcal{Z}, \mathbb{Z})$ is a fixed second homology class. On this moduli space, one can construct a virtual fundamental class of positive degree. Therefore, to obtain numerical invariants, one has to intersect this class with the Euler class of the obstruction bundle $\mathcal{V}=R^{0} \rho_{*}\left(e v^{*} \mathcal{O}\left(-K_{\mathcal{Z}}\right)\right)$. Here $\rho: \bar{M}_{0,1}(\mathcal{Z}, \beta) \longrightarrow \bar{M}_{0,0}(\mathcal{Z}, \beta)$ is the forgetful map and $e v: \bar{M}_{0,1}(\mathcal{Z}, \beta) \longrightarrow \mathcal{Z}$ is the evaluation map as usual. Note that the line bundle $\mathcal{O}\left(-K_{\mathcal{Z}}\right)$ defines the linear system of CalabiYau hypersurfaces on $\mathcal{Z}$. Therefore, the zeroes of a generic section of $\mathcal{V}$ on 
$\bar{M}_{0,0}(\mathcal{Z}, \beta)$ can be thought of intuitively as maps to a generic hypersurface $Y$. In fact, one can show rigorously that these intersection numbers coincide with the degree of the virtual cycle $\left[\bar{M}_{0,0}(Y, \beta)\right]$ (see Example 7.1.5.1 in ref. [12]). Since only the data of the linear system $\left|-K_{\mathcal{Z}}\right|$ enters this definition, the invariants are manifestly independent of complex structure deformations of $Y$. This approach is valid if the bundle $\mathcal{O}\left(-K_{\mathcal{Z}}\right)$ is convex, i.e., $R^{1} \rho_{*}\left(e v^{*} \mathcal{O}\left(-K_{\mathcal{Z}}\right)\right)=0$. By a slight abuse of language, we will call $\mathcal{V}$ a convex obstruction bundle in this case.

Following the same general line of thought, we would like to propose an extension of the convex obstruction bundle approach to open string A-model amplitudes on compact Calabi-Yau hypersurfaces. It should be said at the offset that the methods employed here are not entirely rigorous, since a rigorous mathematical formulation of open string Gromov-Witten invariants has not been developed so far. We make no claim of filling this gap. Moreover, since our approach relies heavily on localization with respect to a toric action, it applies only to a special class of hypersurfaces and Lagrangian cycles which will be described later. The models presented in the next section provide a good testing ground for explicit computations which can be checked against large $N$ duality predictions.

The main idea is quite straightforward. Given a Calabi-Yau hypersurface $Y \subset \mathcal{Z}$ and a collection of vanishing cycles $L=\cup_{i} L_{i}$, we would like to consider the open string morphisms to $Z$ with certain boundary conditions along $L_{i}$, viewed as cycles in $Z$. Obviously, the $L_{i}$ cannot be Lagrangian cycles in $Z$ for dimensional reasons, but this will not be a major obstacle. More precisely, one would like to consider the moduli space of stable maps $\bar{M}_{0, h}(\mathcal{Z}, L ; \beta)$ where $\beta$ denotes now a relative homology class $\beta \in H_{2}(\mathcal{Z}, L ; \mathbb{Z})$. Note that $H_{2}(\mathcal{Z}, L ; \mathbb{Z}) \simeq H_{2}(\mathcal{Z}, \mathbb{Z})$ since $L$ is a collection of 3 -spheres, and hence we can identify $\beta$ with an absolute class. In order to localize the open string morphisms following refs. [25,33, 45], we need a torus action on $\bar{M}_{0, h}(\mathcal{Z}, L ; \beta)$ compatible with the torus action on $\mathcal{Z}$. The problem here is that the torus action on $\mathcal{Z}$ does not preserve the vanishing cycles $L_{i}$ lying on a generic hypersurface $Y$. This is a new element compared to the closed string situation.

In the following, we would like to propose a solution to this problem based on the assumption that toplogical A-model amplitudes are invariant under complex structure deformations. Working under this assumption, it suffices to define and compute these invariants by specializing $(Y, L)$ to a singular hypersurface $\bar{Y}$ and a collection of cycles $\bar{L}$ preserved by a subtorus. The main problem with such an approach is that the existence of such a "good degeneration" of $(Y, L)$ is not by any means obvious. This is in fact the 
main restriction on the class of transitions which can be treated in this fashion. We have not been able to develop a systematic characterization of "good degenerations" in arbitrary families of Calabi-Yau hypersurfaces. Analyzing the concrete examples worked out in the next sections, it seems that such a degeneration will exist whenever the nodal points of $Y_{0}$ are fixed points of the torus action on the ambient toric variety $\mathcal{Z}$. Clearly, this point deserves further study, but this is the criterion that seems to emerge so far.

Provided one can find such a degeneration $\bar{Y}$, the next step is to evaluate the contributions of the fixed loci in $\bar{M}_{0, h}(\mathcal{Z}, \bar{L} ; \beta)$. This can be done by constructing the tangent-obstruction complex for open string morphisms to the pair $(\mathcal{Z}, \bar{L})$. Although the target space is a toric four-fold, the main idea is the same as $[25,33]$, i.e., one has to consider the deformation complex of an open string map $f: \Sigma_{0, h} \longrightarrow \mathcal{Z}$ subject to boundary conditions along $\bar{L}$. The boundary conditions can be inferred from the concrete presentation of $\bar{L}$, as we will discuss in detail in concrete examples. In particular, in all these examples we will find a real subbundle $T_{\mathbb{R}}$ of $T_{\mathcal{Z}}$ restricted to $\bar{L}$ so that the pair $\left(f^{*} T_{\mathcal{Z}}, f_{\partial}^{*} T_{\mathbb{R}}\right)$ forms a Riemann-Hilbert bundle on $\Sigma_{0, h}$.

We need one more ingredient, which is the convex obstruction bundle on the moduli space. One would like to define the fiber of the obstruction bundle $\mathcal{V}$ over a point $\left(\Sigma_{0, h}, f\right)$, as the space of holomorphic sections of $f^{*} \mathcal{O}\left(-K_{\mathcal{Z}}\right)$ subject to certain boundary conditions along $\partial \Sigma_{0, h}$. In order to obtain a good boundary problem for the Dolbeault operator, the boundary conditions should be specified by a real subbundle $\mathcal{R}$ of $\left.f^{*} \mathcal{O}\left(-K_{\mathcal{Z}}\right)\right|_{\partial \Sigma_{0, h}}$ [33]. In fact the pair $\left(f^{*} \mathcal{O}\left(-K_{\mathcal{Z}}\right), \mathcal{R}\right)$ should define a Riemann-Hilbert bundle on $\Sigma_{0, h}$. Such a structure can be naturally obtained in the present case if we use the isomorphism $\mathcal{O}\left(-K_{\mathcal{Z}}\right) \simeq \Lambda^{4}\left(T_{\mathcal{Z}}\right)$, where $T_{\mathcal{Z}}$ is the holomorphic tangent bundle to $\mathcal{Z}$. Then we can take $\mathcal{R}$ to be $f_{\partial}^{*}\left(\Lambda^{4}\left(T_{\mathbb{R}}\right)\right)$. It is not a priori obvious that this construction is sensible, since the structure of the moduli space is not understood. However, we will show in the next sections that the results are in perfect agreement with large $N$ duality predictions. This is a strong evidence in favor of this approach.

As mentioned several times so far, evaluating the contributions of the fixed points is only a first step in the computation of open string amplitudes. In order to finish the computation, the contribution of each fixed point has to be interpreted as a series of instanton corrections in the Chern-Simons theory. This involves another very subtle aspect of the whole approach, namely the correlation between the toric weights and Chern-Simons framing.

Given the complexity of the problem, we will proceed in several stages. In the next section, we describe in detail the models considered in this paper. Although we will find explicit extremal transitions between compact 
Calabi-Yau manifolds, the starting point will be local del Pezzo models. Section 5 will be devoted to the weight-framing correspondence for local models, focusing on the idea of a formal Chern-Simons expansion. Finally, equipped with a better understanding of these points, we will address large $N$ duality for compact models in Sections 6 and 7 .

\section{The models}

Concrete examples in which the above program can be implemented ${ }^{2}$ arise by taking projective completions of the local Calabi-Yau models considered in refs. $[5,15,16]$. Let us briefly recall the relevant geometric constructions.

We will consider extremal transitions for noncompact toric Calabi-Yau three-folds fibered in complex lines over toric del Pezzo surfaces. The base $B$ of the fibration can be either $d P_{2}$ as in the model studied in ref. [16] or $d P_{3}$ or $d P_{5}$ as in ref. [5]. In all cases, it is easier to describe the small resolution $\widetilde{X}$ first, which is isomorphic to the total space of the canonical bundle $\mathcal{O}\left(K_{B}\right)$. The toric diagrams for the del Pezzo surfaces $d P_{2}, d P_{3}$ and $d P_{5}$ are represented below.

In each case, the toric fan of the three-fold $\widetilde{X}$ is a cone over the twodimensional polytope represented in figure 1 . Note that $d P_{2}$ is represented here as a two-point blow-up of $\mathbb{P}^{2}$, and similarly $d P_{3}$ and $d P_{5}$ are constructed by blowing up two and, respectively, four points on $\mathbb{F}_{0}$. In the last case, the four points on $\mathbb{F}_{0}$ are not in general position, and therefore we obtain a nongeneric $d P_{5}$ surface. This is consistent with the toric presentation, since the generic $d P_{5}$ surface is not toric. We denote the exceptional curves by $e_{i}, i=1, \ldots, n$, where $n=2,2,4$ in the three cases, as specified in figure 1 . The $e_{i}$ can be embedded as $(-1,-1)$ curves in $\widetilde{X}$ via the zero section $\sigma: B \longrightarrow \widetilde{X}$.

In the following, we will consider extremal transitions consisting of a contraction of the curves $e_{i}$ on $\widetilde{X}$ followed by a deformation of the resulting nodal singularities. The singular three-folds obtained in the process can be represented as hypersurfaces in noncompact toric varieties $\mathcal{W}[16]$. The toric

\footnotetext{
${ }^{2}$ These are not the only examples which can be treated this way. We will eventually learn along the way that all extremal transitions in which the nodal points are fixed by the torus action admit a similar approach.
} 

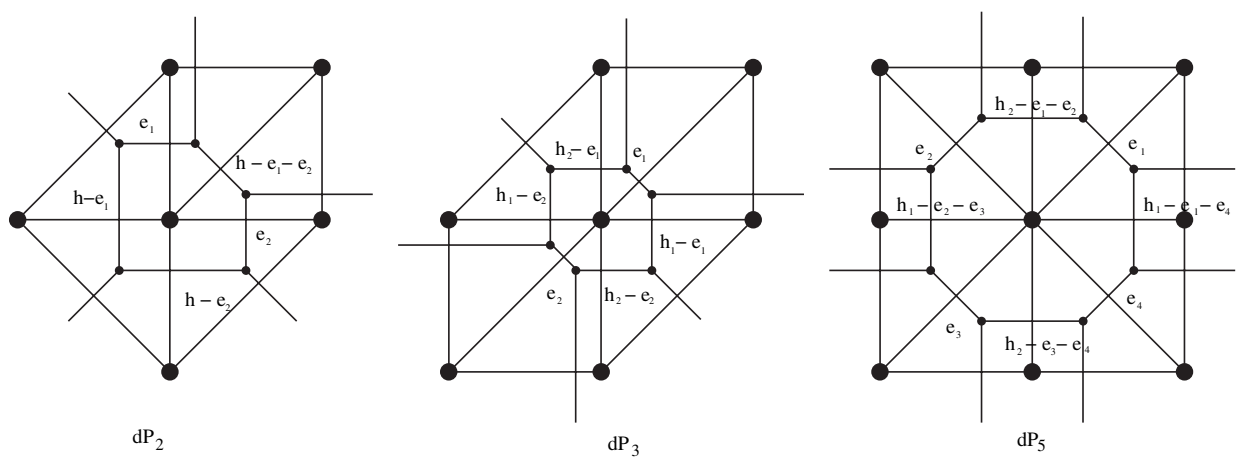

Figure 1: del Pezzo surfaces.

data of $\mathcal{W}$ are

$$
\begin{array}{clcccccc}
d P_{2}: & & Z_{1} & Z_{2} & Z_{3} & U & V & \\
& \mathbb{C}^{*} & 1 & 1 & 1 & -1 & -2 & \\
& & Z_{1} & Z_{2} & Z_{3} & Z_{4} & U & V \\
d P_{3}: & \mathbb{C}^{*} & 1 & 1 & 0 & 0 & -1 & -1 \\
& \mathbb{C}^{*} & 0 & 0 & 1 & 1 & -1 & -1 \\
& & Z_{1} & Z_{2} & Z_{3} & Z_{4} & U & V \\
d P_{5}: & \mathbb{C}^{*} & 1 & 1 & 0 & 0 & -2 & 0 \\
& \mathbb{C}^{*} & 0 & 0 & 1 & 1 & 0 & -2
\end{array} .
$$

The equations for the nodal hypersurfaces read in the three cases

$$
\begin{array}{ll}
d P_{2}: & U Z_{1}+V Z_{2} Z_{3}=0 \\
d P_{3}: & U Z_{1} Z_{4}+V Z_{2} Z_{3}=0 \\
d P_{5}: & U Z_{1} Z_{2}+V Z_{3} Z_{4}=0 .
\end{array}
$$

It is easy to check that the singular points are given by

$$
\begin{array}{lll}
d P_{2}: & P_{1}=\left\{Z_{1}=Z_{2}=U=V=0\right\}, & P_{2}=\left\{Z_{1}=Z_{3}=U=V=0\right\}, \\
d P_{3}: & P_{1}=\left\{Z_{1}=Z_{3}=U=V=0\right\}, & P_{2}=\left\{Z_{2}=Z_{4}=U=V=0\right\}, \\
d P_{5}: & P_{1}=\left\{Z_{1}=Z_{3}=U=V=0\right\}, & P_{2}=\left\{Z_{2}=Z_{4}=U=V=0\right\}, \\
& P_{3}=\left\{Z_{1}=Z_{4}=U=V=0\right\}, & P_{4}=\left\{Z_{2}=Z_{3}=U=V=0\right\} .
\end{array}
$$

The deformations are described by adding a constant term $\mu$ to the right hand side of the equations (4.2). The geometry of the resulting smooth variety can be analyzed in detail as in [16]. In particular, one can show 
that there are $n$ vanishing cycles on the deformed hypersurface $X_{\mu}$ (where $n=2,2,4$ as explained above) subject to one homology relation. These cycles have the topology of a three-sphere and they can be described quite explicitly in terms of local coordinates. We will give more details on this point at a later stage.

Now let us describe the compact Calabi-Yau three-folds associated to the above local models. The idea is quite straightforward. We first take a projective completion of the ambient toric varieties $\mathcal{W}$, obtaining smooth compact toric four-folds $\mathcal{Z}$ given by the following data

$\begin{array}{ccccccccc} & & Z_{1} & Z_{2} & Z_{3} & U & V & W & \\ d P_{2}: & \mathbb{C}^{*} & 1 & 1 & 1 & -1 & -2 & 0 & \\ & \mathbb{C}^{*} & 0 & 0 & 0 & 1 & 1 & 1 & \\ & & Z_{1} & Z_{2} & Z_{3} & Z_{4} & U & V & W \\ d P_{3}: & \mathbb{C}^{*} & 1 & 1 & 0 & 0 & -1 & -1 & 0 \\ & \mathbb{C}^{*} & 0 & 0 & 1 & 1 & -1 & -1 & 0 \\ & \mathbb{C}^{*} & 0 & 0 & 0 & 0 & 1 & 1 & 1 \\ & & Z_{1} & Z_{2} & Z_{3} & Z_{4} & U & V & W \\ d P_{5}: & \mathbb{C}^{*} & 1 & 1 & 0 & 0 & -2 & 0 & 0 \\ & \mathbb{C}^{*} & 0 & 0 & 1 & 1 & 0 & -2 & 0 \\ & \mathbb{C}^{*} & 0 & 0 & 0 & 0 & 1 & 1 & 1\end{array}$

Note that in all cases we obtain a $\mathbb{P}^{2}$ bundle over the base $B$. It is a simple exercise to check that the canonical class of $\mathcal{Z}$ is given by $K_{\mathcal{Z}}=3 \eta$, where $\eta$ is the divisor at infinity defined by the equation $W=0$. The generic divisor in the linear system $\left|-K_{\mathcal{Z}}\right|$ is a smooth Calabi-Yau variety defined by an equation of the form

$$
\sum_{a, b, c \geq 0, a+b+c=3} U^{a} V^{b} W^{c} f_{a b c}\left(Z_{i}\right)
$$

For the $d P_{2}$ model, $f_{a b c}\left(Z_{i}\right)$ is a homogeneous polynomial of degree $a+2 b$ in $\left(Z_{1}, Z_{2}, Z_{3}\right)$. For the other two models, $f_{a b c}\left(Z_{i}\right)$ is a bihomogeneous polynomial of bidegree $(a+b, a+b)$ and, respectively, $(2 a, 2 b)$ in $\left(Z_{1}, Z_{2}\right),\left(Z_{3}, Z_{4}\right)$. One can work out the vertices of the dual toric polyhedra and the Hodge numbers for all the three cases. ${ }^{3}$ The toric polyhedra $\nabla_{Y}$ are specified by

\footnotetext{
${ }^{3}$ The Hodge numbers of all manifolds appearing in this paper have been computed using the program POLYHEDRON, written by Philip Candelas.
} 
the following sets of vertices $\mathcal{V}_{Y}$

$$
\begin{aligned}
& d P_{2}:\{(-1,0,0,0),(-1,1,0,0),(-1,0,1,0),(-1,0,0,1),(-1,1,1,1) \text {, } \\
& (2,-1,-1,-1)\} \text {, } \\
& d P_{3}: \quad\{(0,1,0,0),(0,0,1,0),(0,0,0,1),(1,-1,0,0),(1,0,-1,0) \text {, } \\
& (1,0,0,-1),(-1,0,0,0)\} \\
& d P_{5}: \quad\{(1,1,0,0),(1,-1,0,1),(-1,1,-1,0),(-1,-1,1,0) \text {, } \\
& (-1,-1,0,1)\} \text {. }
\end{aligned}
$$

The Hodge numbers of the three models are, respectively, $\left(h_{1,1}, h_{1,2}\right)=$ $(2,92),(3,81),(3,63)$.

The local extremal transitions can be lifted to transitions between compact Calabi-Yau three-folds as follows. The one-parameter families $Y_{\mu}$ are given by the following equations

$$
\begin{aligned}
& \left(U Z_{1}+V Z_{2} Z_{3}-\mu W\right) W^{2}+\sum_{(a, b, c)^{\prime}} U^{a} V^{b} W^{c} f_{a b c}\left(Z_{i}\right)=0 \\
& \left(U Z_{1} Z_{4}+V Z_{2} Z_{3}-\mu W\right) W^{2}+\sum_{(a, b, c)^{\prime}} U^{a} V^{b} W^{c} f_{a b c}\left(Z_{i}\right)=0, \\
& \left(U Z_{1} Z_{2}+V Z_{3} Z_{4}-\mu W\right) W^{2}+\sum_{(a, b, c)^{\prime}} U^{a} V^{b} W^{c} f_{a b c}\left(Z_{i}\right)=0
\end{aligned}
$$

where the $(a, b, c)^{\prime}$ denote all the allowed triples $(a, b, c)$ which do not appear in the first three terms in each equation. The coefficients of the polynomials $f_{a b c}\left(Z_{i}\right)$ are fixed at some generic values so that we obtain one-parameter families parameterized by $\mu$. These hypersurfaces are smooth for $\mu=0$ and develop isolated nodal singularities at $\mu=0$. The singular points are again given by equation (4.3), except that they have to be regarded as points on the compact toric four-fold $\mathcal{Z}$. These singularities can be simultaneously resolved by performing a blow-up of $\mathcal{Z}$ along the section $U=V=0$. Let $\widetilde{\mathcal{Z}}$ denote the resulting toric four-fold. The strict transform $\widetilde{Y} \subset \widetilde{\mathcal{Z}}$ is a crepant resolution of $Y_{0}$ with exceptional locus given by isolated $(-1,-1)$ curves $C_{i}$, $i=1, \ldots, n$.

The blow-up of the ambient toric variety can be described torically by adding an extra vertex to the dual toric polyhedron $\nabla_{Y}$. One then obtains the following toric data for the dual polyhedron of the small resolution $\widetilde{Y}$

$$
\begin{array}{ll}
d P_{2}: & \mathcal{V}_{\widetilde{Y}}=\mathcal{V}_{Y} \cup\{(-2,1,1,1)\}, \\
d P_{3}: & \mathcal{V}_{\widetilde{Y}}=\mathcal{V}_{Y} \cup\{(1,0,0,0)\}, \\
d P_{5}: & \mathcal{V}_{\widetilde{Y}}=\mathcal{V}_{Y} \cup\{(-1,-1,0,0)\}
\end{array}
$$


The package PALP [37] proved to be very useful in analyzing the above extremal transitions between the smooth three-folds $Y$ and $\widetilde{Y}$. Moreover, the Hodge numbers of $\widetilde{Y}$ are, respectively, $\left(h_{1,1}, h_{1,2}\right)=(3,91),(4,80),(4,60)$ for the three cases. In the following sections, we will develop in detail a computational approach to open string topological amplitudes on the threefolds $Y$. Although we will work out the details only for the second model presented above, it is clear that the other models can be treated along the same lines. Moreover, these techniques seem to be valid for all extremal transitions in which the singular points are fixed under the generic torus action on $\mathcal{Z}$.

\section{Local transitions and formal Chern-Simons expansion}

Following the program outlined in Section 3, here we review the open string localization techniques for local transitions developed in refs. $[15,16]$. The main goal of this review is to clarify the interplay between torus action and framing in Chern-Simons theory. We will find that the proper framework for open string amplitudes is a formal extension of the Chern-Simons expansion which treats the framing as a formal variable. This new idea will allow us to formulate general rules for the correspondence between toric weights and framing encompassing all cases known so far $[5,15,16]$. Moreover, as discussed in Section 6, the same rules play a crucial role for open string amplitudes on compact manifolds. Let us start with the first local model described in the previous section, namely the local $d P_{2}$ model. The deformed hypersurface $X_{\mu}$ is given by the equation

$$
U Z_{1}+V Z_{2} Z_{3}=\mu
$$

in the toric variety $\mathcal{W}$ specified in equation (4.1), and the singular points at $\mu=0$ are $P_{1}=\left\{Z_{1}=Z_{2}=U=V=0\right\}$ and $P_{2}=\left\{Z_{1}=Z_{3}=U=V=\right.$ $0\}$. The geometry of this model has been thoroughly analyzed in ref. [16], so we will keep the details to a minimum. The main point is that there are two Lagrangian spheres $L_{1}$ and $L_{2}$ on $X_{\mu}$ in the same homology class $\left[L_{1}\right]=\left[L_{2}\right]$. Each cycle can be described as an intersection of $X_{\mu}$ with two real hypersurfaces in $\mathcal{W}$ given by the following equations

$$
\begin{aligned}
& L_{1}: \quad U Z_{2} \bar{Z}_{2}-W \bar{Z}_{1}=0, \quad V Z_{2}^{2} \bar{Z}_{2}-W \bar{Z}_{3}=0, \\
& L_{2}: \quad U Z_{3} \bar{Z}_{3}-W \bar{Z}_{1}=0, \quad V Z_{3}^{2} \bar{Z}_{3}-W \bar{Z}_{2}=0 .
\end{aligned}
$$

Writing these equations in suitable local coordinates centered at the points $P_{1}, P_{2} \in \mathcal{W}$, one can check that the local geometry is indeed isomorphic to a local deformation of a conifold singularity. Moreover, it has been shown in ref. [16] that one can choose a symplectic Kähler structure on $\mathcal{W}$ so that $L_{1}$ and $L_{2}$ are Lagrangian. 
The open string topological A-model considered in ref. [16] is defined by wrapping $N_{1}$ and $N_{2}$ topological branes on $L_{1}$ and $L_{2}$. As explained there, and also in Section 3 of this paper, a crucial step in the computation of the free energy is summing up the instanton corrections to Chern-Simons theory. This can be done by localization with respect to the following toric action

$$
\begin{array}{ccccccc} 
& Z_{1} & Z_{2} & Z_{3} & U & V & W \\
S^{1} & \lambda_{1} & \lambda_{2} & 0 & -\lambda_{1} & -\lambda_{2} & 0
\end{array} .
$$

Note that this action preserves both the hypersurface $X_{\mu}$ and the spheres $L_{1}$ and $L_{2}$. We have to sum over open string maps which are invariant under the induced toric action on the moduli space. As usual in localization computations, the image of all such maps consists of a collection of invariant holomorphic Riemann surfaces embedded in $X_{\mu}$. In closed string situations, these would have to be closed Riemann surfaces, or equivalently algebraic curves on $X_{\mu}$. Since we are doing an open string computation, we will have a collection of invariant bordered Riemann surfaces with boundary components embedded in $L_{1}$ and $L_{2}$. According to the analysis of Diaconescu et al. [16], there are only three such invariant surfaces - two discs and a cylinder-which can be described as follows.

Let $\bar{X}_{\mu}$ be the (relative) projective closure of $X_{\mu}$ in the compact toric variety $\mathcal{Z}$. The defining equation of $\bar{X}_{\mu}$ is

$$
U Z_{1}+V Z_{2} Z_{3}=\mu W
$$

One can check that $\bar{X}_{\mu}$ is a toric Fano three-fold which contains $X_{\mu}$ as the complement of the divisor at infinity $D_{\infty}=\bar{X}_{\mu} \cap\{W=0\}$. The bordered Riemann surfaces are obtained by intersecting the spheres $L_{1}$ and $L_{2}$ with three invariant curves on $\bar{X}_{\mu}$ given by

$$
\begin{array}{ll}
C_{1}^{\prime}: \quad V=Z_{2}=0, \quad U Z_{1}=\mu W, \\
C_{2}^{\prime}: \quad V=Z_{3}=0, \quad U Z_{1}=\mu W, \\
C_{3}^{\prime}: \quad U=Z_{1}=0, \quad V Z_{2} Z_{3}=\mu W .
\end{array}
$$

We have the following intersections $L_{1} \cap C_{1}^{\prime}=\Gamma_{1}^{\prime}, L_{2} \cap C_{2}^{\prime}=\Gamma_{2}^{\prime}, L_{1} \cap C_{3}^{\prime}=$ $\Xi_{1}^{\prime}$ and $L_{2} \cap C_{3}^{\prime}=\Xi_{2}^{\prime}$, where $\Gamma_{1}^{\prime}$ and $\Xi_{1}^{\prime}$ and, respectively, $\Gamma_{2}^{\prime}$ and $\Xi_{2}^{\prime}$ are algebraic knots in $L_{1}$ and $L_{2}$ forming Hopf links with linking number +1 . $\Gamma_{1}^{\prime}$ divides $C_{1}^{\prime}$ into two invariant discs $D_{1}^{\prime}$ and $D_{1}^{\prime \prime}$ and $\Gamma_{2}^{\prime}$ divides $C_{2}^{\prime}$ into two other invariant discs $D_{2}^{\prime}$ and $D_{2}^{\prime \prime}$. Similarly, $\Xi_{1}^{\prime}$ and $\Xi_{2}^{\prime}$ divide $C_{3}^{\prime}$ into two discs $D_{3}^{\prime \prime}$ and $D_{4}^{\prime \prime}$ and a holomorphic annulus $A$ as in figure 2 .

Note that the discs $D_{1}^{\prime}$ and $D_{2}^{\prime}$ have a common origin at $Q=\left\{Z_{2}=Z_{3}=\right.$ $\left.V=0, U Z_{1}=\mu W\right\}$. The origins of all other discs are points on the divisor at infinity $D_{\infty}$. 


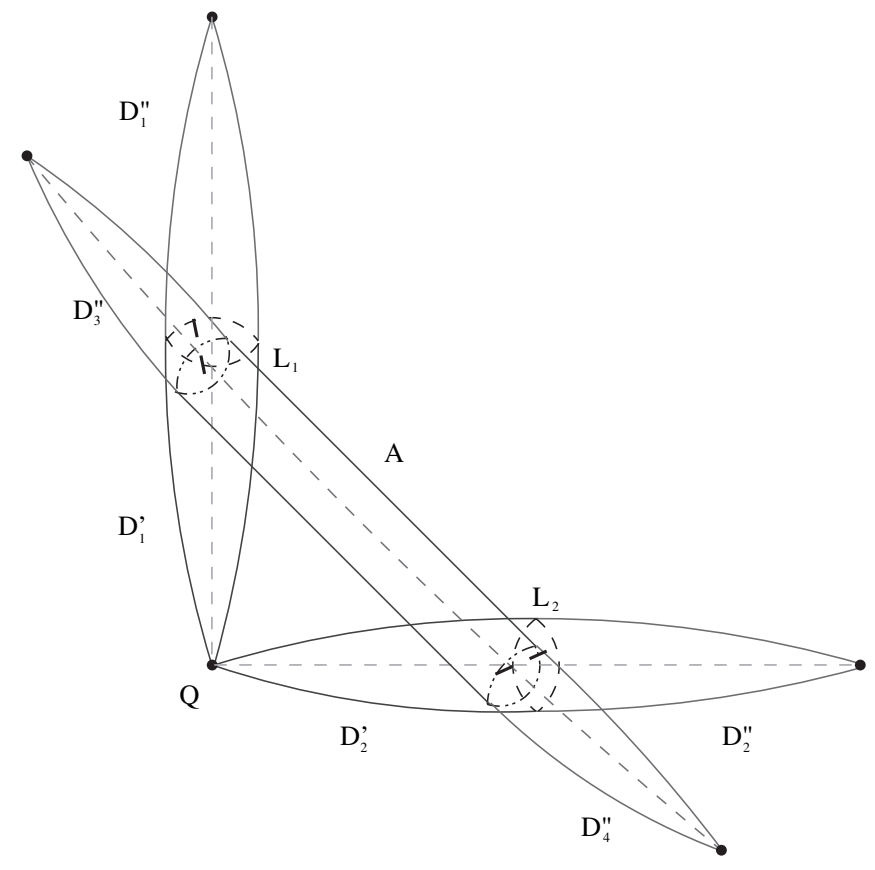

Figure 2: Primitive open string instantons on $X$.

A careful argument based on homology constraints [16] shows that only $D_{1}^{\prime}, D_{2}^{\prime}$ and $A$ contribute to the open string amplitudes in the local case. The other discs do not play any role for the local computation, but they will enter the computation of open string amplitudes for the compact model. In order to write down a general formula for the instanton corrections, let us denote the holonomy variables associated to the four knots $\Gamma_{1}^{\prime}, \Gamma_{2}^{\prime}, \Xi_{1}^{\prime}$ and $\Xi_{2}^{\prime}$ by $V_{1}^{\prime}, V_{2}^{\prime}, U_{1}^{\prime}$ and $U_{2}^{\prime}$, respectively. The instanton series has the form

$$
\begin{aligned}
& F_{\text {inst }}\left(g_{\mathrm{s}}, t_{1}^{\prime}, t_{2}^{\prime}, t_{c}, U_{1}^{\prime}, U_{2}^{\prime}, V_{1}^{\prime}, V_{2}^{\prime}\right) \\
& \quad=F_{\text {inst }}^{(1)}\left(g_{\mathrm{s}}, t_{c}, U_{1}^{\prime}, U_{2}^{\prime}\right)+F_{\text {inst }}^{(2)}\left(g_{\mathrm{s}}, t_{1}^{\prime}, t_{2}^{\prime}, V_{1}^{\prime}, V_{2}^{\prime}\right),
\end{aligned}
$$

where

$$
\begin{aligned}
F_{\text {inst }}^{(1)}\left(g_{\mathrm{s}}, t_{c}, U_{1}^{\prime}, U_{2}^{\prime}\right)= & \sum_{g=0}^{\infty} \sum_{h_{1}, h_{2}=0}^{\infty} \sum_{d=0}^{\infty} \sum_{m_{i} \geq 0, n_{j} \geq 0} i^{h_{1}+h_{2}} g_{\mathrm{s}}^{2 g-2+h_{1}+h_{2}} \\
& \times C_{g, h_{1}, h_{2}}\left(d \mid m_{i}, n_{j}\right) \mathrm{e}^{-d t_{c}} \prod_{i=1}^{h_{1}} \operatorname{Tr} U_{1}^{\prime m_{i}} \prod_{j=1}^{h_{2}} \operatorname{Tr} U_{2}^{\prime n_{j}},
\end{aligned}
$$




$$
\begin{aligned}
F_{\text {inst }}^{(2)}\left(g_{\mathrm{s}}, t_{1}^{\prime}, t_{2}^{\prime}, V_{1}^{\prime}, V_{2}^{\prime}\right)= & \sum_{g=0}^{\infty} \sum_{h_{1}, h_{2}=0}^{\infty} \sum_{d_{1}, d_{2}=0}^{\infty} \sum_{m_{i} \geq 0, n_{j} \geq 0} i^{h_{1}+h_{2}} g_{\mathrm{s}}^{2 g-2+h_{1}+h_{2}} \\
& \times F_{g, h_{1}, h_{2}}\left(d_{1}, d_{2} \mid m_{i}, n_{j}\right) \mathrm{e}^{-d_{1} t_{1}^{\prime}-d_{2} t_{2}^{\prime}} \\
& \times \prod_{i=1}^{h_{1}} \operatorname{Tr} V_{1}^{\prime m_{i}} \prod_{j=1}^{h_{2}} \operatorname{Tr} V_{2}^{\prime n_{j}}
\end{aligned}
$$

The first represents the contribution of multicovers of the cylinder $A$ and the second term represents the contribution of invariant maps with image contained in the union $D_{1}^{\prime} \cup D_{2}^{\prime}$. The parameters $t_{1}^{\prime}, t_{2}^{\prime}$ and $t_{c}$ represent open string Kähler moduli. At the classical level, they should equal the symplectic areas of $D_{1}^{\prime}, D_{2}^{\prime}, A$ which are equal since all these surfaces are homologically equivalent. However, it is understood by now that these parameters can receive quantum corrections due to degenerate instantons, which can be in principle different for the three surfaces. The rest of the notation is standard: $d, d_{1}$ and $d_{2}$ denote the degrees of a given map with respect to the three fixed surfaces $A, D_{1}^{\prime}$ and $D_{2}^{\prime}$ and $\left(m_{i}, n_{j}\right)$ denote the winding numbers of the boundary components about the invariant knots in the target space.

The coefficients $C_{g, h_{1}, h_{2}}\left(d \mid m_{i}, n_{j}\right)$ and $F_{g, h_{1}, h_{2}}\left(d_{1}, d_{2} \mid m_{i}, n_{j}\right)$ can be determined by localization computations for open string morphisms. This computation has been done in great detail in ref. [16], and we will not repeat it here. For our purposes, it suffices to recall the results for genus zero maps of total degree smaller or equal than 3 . We will rewrite all the formulae obtained in ref. [16], in terms of the ratio of toric weights $z=\lambda_{2} / \lambda_{1}$.

Let us start with multicovers of the two discs. Since we do not make a particular choice of toric weights, we will have nonzero corrections corresponding to surfaces with one, two and three boundary components. We have the following expressions.

Degree 1:

$$
F_{0,1,0}(1,0 \mid 1)=F_{0,0,1}(0,1 \mid 1)=1 .
$$

Degree 2:

$$
\begin{aligned}
& F_{0,1,0}(2,0 \mid 2)=\frac{2 z+1}{4}, \\
& F_{0,0,1}(0,2 \mid 2)=\frac{1-z}{4(1+z)}, \\
& F_{0,2,0}(2,0 \mid 1,1)=-\frac{z(1+z)}{4}, \\
& F_{0,0,2}(0,2 \mid 1,1)=\frac{z}{4(1+z)^{2}}, \\
& F_{0,1,1}(1,1 \mid 1,1)=-1 .
\end{aligned}
$$


Degree 3:

$$
\begin{aligned}
& F_{0,1,0}(3,0 \mid 3)=\frac{(3 z+1)(3 z+2)}{18}, \\
& F_{0,0,1}(0,3 \mid 3)=\frac{(1-2 z)(2-z)}{18(1+z)^{2}}, \\
& F_{0,1,1}(2,1 \mid 2,1)=-z, \\
& F_{0,1,1}(1,2 \mid 1,2)=\frac{z}{1+z}, \\
& F_{0,2,1}(2,1 \mid 1,1,1)=\frac{z^{2}}{2}, \\
& F_{0,1,2}(1,2 \mid 1,1,1)=\frac{z^{2}}{2(1+z)^{2}}, \\
& F_{0,2,0}(3,0 \mid 2,1)=-\frac{z(z+1)(2 z+1)}{3}, \\
& F_{0,0,2}(0,3 \mid 2,1)=\frac{z(1-z)}{3(1+z)^{3}}, \\
& F_{0,3,0}(3,0 \mid 1,1,1)=\frac{z^{2}(1+z)^{2}}{6}, \\
& F_{0,0,3}(0,3 \mid 1,1,1)=\frac{z^{2}}{6(1+z)^{4}} .
\end{aligned}
$$

The multicover contributions of the cylinder are independent of toric weights. In fact, one has a very simple formula of the form

$$
C_{0,1,1}(d \mid d, d)=\frac{(-1)^{d}}{d}
$$

for multicovers of degree $d$ and all other terms being zero. The alternating sign in this formula needs some explanation. A common problem with open string localization computations is that the moduli spaces of open string maps, and the obstruction bundles do not have a canonical complex structure as in the closed string case. Instead, one has only a complex structure up to conjugation. This gives rise to a sign ambiguity in the open string amplitudes noticed first time in refs. [25,33]. In the absence of a rigorous framework for these computations, this ambiguity cannot be resolved from first principles. Since the present open string model has a closed string dual, a practical solution to this problem is to fix the sign so that the final results agree with the closed string amplitudes. This is not a satisfactory solution, but it seems to be the only one available at the moment. The choice made here is different from the one made in ref. [16], where the sign was taken 
to be +1 for all $d$. It will be clear shortly that equation (5.12) is a more natural choice in this context.

The next step is to perform the Chern-Simons functional integral taking into account the instanton corrections (5.9)-(5.12). At this stage, we need to make a choice of framing for the knots $\Gamma_{1}^{\prime}, \Gamma_{2}^{\prime}, \Xi_{1}^{\prime}$ and $\Xi_{2}^{\prime}$. The idea proposed in ref. [16] is that this choice is related to a choice of toric weights, namely to the value of $z$. Let us discuss some of the main points. Choose local coordinates $\left(x_{1}, y_{1}, u_{1}, v_{1}\right)$ centered at $Z_{1}=Z_{2}=U=V=0$ and $\left(x_{2}, y_{2}, u_{2}, v_{2}\right)$ centered at $Z_{1}=Z_{3}=U=V=0$. The local equations of the three-fold $X_{\mu}$ in these patches are

$$
x_{1} u_{1}+y_{1} v_{1}=\mu, \quad x_{2} u_{2}+y_{2} v_{2}=\mu,
$$

and the spheres $L_{1}$ and $L_{2}$ are described by the real sections

$$
L_{1}: \quad u_{1}=\bar{x}_{1}, \quad v_{1}=\bar{y}_{1}, \quad L_{2}: \quad u_{2}=\bar{x}_{2}, \quad v_{2}=\bar{y}_{2} .
$$

The knots $\Gamma_{1}^{\prime}, \Xi_{1}^{\prime} \subset L_{1}$ are cut by the equations $y_{1}=v_{1}=0$ and, respectively, $x_{1}=u_{1}=0$ on $L_{1}$. In order to specify a framing of $\Gamma_{1}^{\prime}$ and $\Xi_{1}^{\prime}$ we have to specify sections of the normal bundles $N_{\Gamma_{1}^{\prime} / L_{1}}$ and $N_{\Xi_{1}^{\prime} / L_{1}}$, respectively. Let us parameterize the knots by the angular variables $\theta_{x_{1}}$ and $\theta_{y_{1}}$ corresponding to the complex coordinates $x_{1}$ and $y_{1}$, respectively. Then we can write down the normal sections in the form

$$
\begin{aligned}
& \Gamma_{1}^{\prime}: \quad\left(y_{1}, v_{1}\right)=\left(\mu^{1 / 2} \mathrm{e}^{\mathrm{i} p_{1} \theta_{x_{1}}}, \mu^{1 / 2} \mathrm{e}^{-\mathrm{i} p_{1} \theta_{x_{1}}}\right), \\
& \Xi_{1}^{\prime}: \quad\left(x_{1}, u_{1}\right)=\left(\mu^{1 / 2} \mathrm{e}^{\mathrm{i} q_{1} \theta_{y_{1}}}, \mu^{1 / 2} \mathrm{e}^{-\mathrm{i} q_{1} \theta_{y_{1}}}\right),
\end{aligned}
$$

where $\left(p_{1}, q_{1}\right)$ determine the framing of the two knots. ${ }^{4}$ We can write down analogous formulae for the pair $\left(\Gamma_{2}^{\prime}, \Xi_{2}^{\prime}\right)$ of knots in $L_{2}$

$$
\begin{aligned}
& \Gamma_{2}^{\prime}: \quad\left(y_{2}, v_{2}\right)=\left(\mu^{1 / 2} \mathrm{e}^{\mathrm{i} p_{2} \theta_{x_{2}}}, \mu^{1 / 2} \mathrm{e}^{-\mathrm{i} p_{2} \theta_{x_{2}}}\right), \\
& \Xi_{2}^{\prime}: \quad\left(x_{2}, u_{2}\right)=\left(\mu^{1 / 2} \mathrm{e}^{\mathrm{i} q_{2} \theta_{y_{2}}}, \mu^{1 / 2} \mathrm{e}^{-\mathrm{i} q_{2} \theta_{y_{2}}}\right) .
\end{aligned}
$$

The relation between the framings $\left(p_{1}, q_{1}\right),\left(p_{2}, q_{2}\right)$ and the toric weights follows by imposing the condition that equations (5.15) and (5.16) be preserved by the torus action, as first proposed in ref. [33]. This yields the

\footnotetext{
${ }^{4}$ There is a subtlety here pointed out in ref. [16]. Namely, specifying a single section of a principal $S^{1}$ bundle over $S^{1}$ does not determine an integer number. One needs to specify in fact a pair of sections in order to obtain integral data. In the present case, there is a canonical choice for a reference section since we have explicitly constructed $\left(\Gamma_{1}^{\prime}, \Xi_{1}^{\prime}\right)$ as algebraic knots. This choice determines the framing to be $\left(p_{1}, q_{1}\right)$.
} 
following relations

$$
\begin{array}{ll}
\lambda_{y_{1}}=p_{1} \lambda_{x_{1}}, & \lambda_{x_{1}}=q_{1} \lambda_{y_{1}}, \\
\lambda_{y_{2}}=p_{2} \lambda_{x_{2}}, & \lambda_{x_{2}}=q_{2} \lambda_{y_{2}},
\end{array}
$$

where the $\left(\lambda_{x_{1}}, \ldots, \lambda_{y_{2}}\right)$ denote the weights of the torus action on the local coordinates, which can be easily expressed in terms of $\left(\lambda_{1}, \lambda_{2}\right)$. This is a very constrained system of equations, since $\left(p_{1}, \ldots, q_{2}\right)$ have to be integers in order for the solution to make sense within the framework of standard Chern-Simons theory. In fact, it is not hard to show that there are no nontrivial solutions satisfying all these conditions. The solution proposed in ref. [16] was to impose these conditions only for the knots $\Gamma_{1}^{\prime}$ and $\Gamma_{2}^{\prime}$, i.e., the boundary components of the two discs $D_{1}^{\prime}$ and $D_{2}^{\prime}$. Then, the framings $\left(q_{1}, q_{2}\right)$ of $\left(\Xi_{1}^{\prime}, \Xi_{2}^{\prime}\right)$ were determined by more indirect arguments to be $(0,0)$. Furthermore, imposing the equivariance conditions for the sections of $N_{\Gamma_{1}^{\prime} / L_{1}}$ and $N_{\Gamma_{2}^{\prime} / L_{2}}$ leaves us with only two sensible solutions

$$
\begin{aligned}
& p_{1}=p_{2}=2 \Rightarrow z=0, \\
& p_{1}=p_{2}=0 \Rightarrow z=1 .
\end{aligned}
$$

The first solution was shown to be a sensible choice in ref. [16], although there were some unclear aspects of the duality map. We will shortly come back to this point.

Here we would like to propose a different solution to this problem which involves far less obscure choices. The main idea is to relax the condition that the framings $\left(p_{1}, q_{1}, p_{2}, q_{2}\right)$ be integral. Instead, our proposal is to treat them as formal variables which can in particular take fractional values. Although this idea seems to be at odds with standard Chern-Simons theory, we will show below that it makes perfect sense provided one treats the vacuum expectation values of Wilson loops as formal power series expansion in the framing variables. Following this idea, we can easily solve all equivariance conditions (5.17)

$$
p_{1}=z+2, \quad q_{1}=\frac{1}{z+2}, \quad p_{2}=\frac{z+2}{z+1}, \quad q_{2}=\frac{z+1}{z+2},
$$

where $z$ is left undetermined. This fixes the framing of all knots in the problem with no ambiguities. At the same time, the instanton corrections (5.9)-(5.11) also depend on the variable $z$. The next step is to perform the Chern-Simons computations treating $z$ as a formal variable and expanding all knot and link invariants in powers of $z$. The truly remarkable aspect of this procedure is that the final result turns out to be independent of $z$, as it is usually the case with closed string localization computations! Below we will perform the computations for genus zero amplitudes up to degree 3 , finding very strong evidence for this conjecture. 
For concreteness, let us collect the instanton corrections (5.9)-(5.12) in a single formula

$$
\begin{aligned}
F_{\text {inst }}= & \frac{\mathrm{i} q_{1}^{\prime}}{g_{\mathrm{s}}} \operatorname{Tr} V_{1}^{\prime}+\frac{\mathrm{i} q_{2}^{\prime}}{g_{\mathrm{s}}} \operatorname{Tr} V_{2}^{\prime}-q_{c} \operatorname{Tr} U_{1}^{\prime} \operatorname{Tr} U_{2}^{\prime}+\frac{\mathrm{i} q_{1}^{\prime 2}}{g_{\mathrm{s}}} \frac{2 z+1}{4} \operatorname{Tr} V_{1}^{\prime 2} \\
& +\frac{\mathrm{i} q_{2}^{\prime 2}}{g_{\mathrm{s}}} \frac{(1-z)}{4(1+z)} \operatorname{Tr} V_{2}^{\prime 2}+q_{1}^{\prime 2} \frac{z(1+z)}{4}\left(\operatorname{Tr} V_{1}^{\prime}\right)^{2}-q_{2}^{\prime 2} \frac{z}{4(1+z)^{2}}\left(\operatorname{Tr} V_{2}^{\prime}\right)^{2} \\
& +q_{1}^{\prime} q_{2}^{\prime} \operatorname{Tr} V_{1}^{\prime} \operatorname{Tr} V_{2}^{\prime}+\frac{1}{2} q_{c}^{2} \operatorname{Tr} U_{1}^{\prime 2} \operatorname{Tr} U_{2}^{\prime 2} \\
& +\frac{\mathrm{i} q_{1}^{\prime 3}}{g_{\mathrm{s}}} \frac{(3 z+1)(3 z+2)}{18} \operatorname{Tr} V_{1}^{\prime 3}+\frac{\mathrm{i} q_{2}^{\prime 3}}{g_{\mathrm{s}}} \frac{(1-2 z)(2-z)}{18(1+z)^{2}} \operatorname{Tr} V_{2}^{\prime 3} \\
& +q_{1}^{\prime 2} q_{2}^{\prime} z \operatorname{Tr} V_{1}^{\prime 2} \operatorname{Tr} V_{2}^{\prime}-q_{1}^{\prime} q_{2}^{\prime 2} \frac{z}{1+z} \operatorname{Tr} V_{1}^{\prime} \operatorname{Tr} V_{2}^{\prime 2} \\
& +q_{1}^{\prime 3} \frac{z(z+1)(2 z+1)}{3} \operatorname{Tr} V_{1}^{\prime 2} \operatorname{Tr} V_{1}^{\prime}-q_{2}^{\prime 3} \frac{z(1-z)}{3(1+z)^{3}} \operatorname{Tr} V_{2}^{\prime 2} \operatorname{Tr} V_{2}^{\prime} \\
& -\mathrm{i} g_{\mathrm{s}} q_{1}^{\prime 2} q_{2}^{\prime} \frac{z^{2}}{2}\left(\operatorname{Tr} V_{1}^{\prime}\right)^{2} \operatorname{Tr} V_{2}^{\prime}-\mathrm{i} g_{\mathrm{s}} q_{1}^{\prime} q_{2}^{\prime 2} \frac{z^{2}}{2(1+z)^{2}} \operatorname{Tr} V_{1}^{\prime}\left(\operatorname{Tr} V_{2}^{\prime}\right)^{2} \\
& -\mathrm{i} g_{\mathrm{s}} q_{1}^{\prime 3} \frac{z^{2}(1+z)^{2}}{6}\left(\operatorname{Tr} V_{1}^{\prime}\right)^{3}-\mathrm{i} g_{\mathrm{s}} q_{2}^{\prime 3} \frac{z^{2}}{6(1+z)^{4}}\left(\operatorname{Tr} V_{2}^{\prime}\right)^{3} \\
& -\frac{1}{3} q_{c}^{3} \operatorname{Tr} U_{1}^{\prime 3} \operatorname{Tr} U_{2}^{\prime 3} .
\end{aligned}
$$

In this formula, $q_{1}^{\prime}=\mathrm{e}^{-t_{1}^{\prime}}, q_{2}^{\prime}=\mathrm{e}^{-t_{2}^{\prime}}$ and $q_{c}=\mathrm{e}^{-t_{c}}$ denote the instanton factors associated to the three primitive open string instantons.

The free energy of the topological open string is given by the following formula

$$
\mathcal{F}_{\text {op }}\left(g_{\mathrm{s}}, q_{1}^{\prime}, q_{2}^{\prime}, q_{c}, \lambda_{1}, \lambda_{2}\right)=\mathcal{F}_{1}^{\mathrm{CS}}\left(\lambda_{1}, g_{\mathrm{s}}\right)+\mathcal{F}_{2}^{\mathrm{CS}}\left(\lambda_{2}, g_{\mathrm{s}}\right)+\ln \left\langle\mathrm{e}^{F_{\text {inst }}}\right\rangle
$$

where $\lambda_{1}$ and $\lambda_{2}$ are the 't Hooft couplings of the two Chern-Simons theories, which are related to the string coupling by $\lambda_{1,2}=N_{1,2} g_{\mathrm{s}}$. The first two terms in the right-hand side of this equation represent the contributions of the two Chern-Simons sectors while the third term represents the expectation value of the instanton corrections. In the following, we will concentrate on the last term. As mentioned above, our goal is to show that the resulting expression for the free energy agrees with the closed string expansion of the local $d P_{2}$ model. In particular, it should be independent of $z$. Similar computations have been performed in ref. [16], so we will not repeat all the details here. The important point in the present approach is that all knot invariants occurring in the process have to be expanded as formal power series of the framing variables. 
Let us explain the algorithm for a general link $\mathcal{L}$ with $c$ components $R_{\alpha}$ and framings $p_{\alpha}, \alpha=1, \ldots, c$. The framing dependence of the expectation value $\left\langle W_{R_{\alpha}}(\mathcal{L})\right\rangle$ is of the form

$$
\left\langle W_{R_{\alpha}}(\mathcal{L})\right\rangle_{\left(p_{1}, \ldots, p_{c}\right)}=e^{\left(i g_{\mathrm{s}} / 2\right) \sum_{\alpha=1}^{c} \kappa_{R_{\alpha}} p_{\alpha}} \mathrm{e}^{(i \lambda / 2) \sum_{\alpha=1}^{c} l_{\alpha} p_{\alpha}}\left\langle W_{R_{\alpha}}(\mathcal{L})\right\rangle_{(0, \ldots, 0)},
$$

where $l_{\alpha}$ is the total number of boxes in the Young tableau of $R_{\alpha}$, and $\kappa_{R_{\alpha}}$ is a group-theoretic quantity defined as follows. Let $v=1, \ldots, r$ label the rows of the Young tableau of a representation $R$ and $l_{v}$ denote the length of the $v$ th row. Then we have [47]

$$
\kappa_{R}=l+\sum_{v=1}^{r}\left(l_{v}^{2}-2 v l_{v}\right),
$$

where $l=\sum_{v=1}^{r} l_{v}$ is the total number of boxes. In the process of evaluating equation (5.21) we will encounter trace products of the form $\prod_{i} \operatorname{Tr} V^{n_{i}}$, where $V$ is any of the holonomy variables $V_{1}^{\prime}, V_{2}^{\prime}, U_{1}^{\prime}$ and $U_{2}^{\prime}$. Such products have to be written as linear combinations of the form $\sum_{R} \operatorname{Tr}_{R} V$ in order to evaluate the Chern-Simons vacuum expectation values. A general rule in all such cases is that the number of boxes in the Young diagram of any representation $R$ in this sum equals the total degree $d=\sum_{i} n_{i}$ of the open string map. All the resulting terms will be weighted by $q^{d}$, where $q=q_{1}^{\prime}, q_{2}^{\prime}$ or $q_{c}$ is the appropriate instanton factor. This means that we will always obtain combinations of the form $\left(q \mathrm{e}^{\mathrm{i}(\lambda / 2) p}\right)^{d}$ in the final expression for the free energy (where $p$ is the framing of the appropriate knot). Therefore, we can simplify the computation by absorbing all the prefactors $\mathrm{e}^{(\mathrm{i} \lambda / 2) \sum_{\alpha=1}^{c} l_{\alpha} p_{\alpha}}$ in a redefinition of the instanton factors. Since these factors are independent of $g_{\mathrm{s}}$ and do not play any role in the expansion, we will call them trivial framing factors. With this redefinition understood, we can use the following expression for the link invariants in the Chern-Simons expansion

$$
\left\langle W_{R_{\alpha}}(\mathcal{L})\right\rangle_{\left(p_{1}, \ldots, p_{c}\right)}^{\prime}=\mathrm{e}^{\left(\mathrm{i} g_{\mathrm{s}} / 2\right) \sum_{\alpha=1}^{c} \kappa_{R_{\alpha}} p_{\alpha}}\left\langle W_{R_{\alpha}}(\mathcal{L})\right\rangle_{(0, \ldots, 0)} .
$$

As explained above, all the expectation values must be written as power series in the framing variables, which appear in the exponential prefactor of equation (5.23) multiplying the string coupling $g_{\mathrm{s}}$. Since we want to obtain the result as a power series in $g_{\mathrm{s}}$, the most efficient way to proceed is to expand all expectation values equation (5.23) in $g_{\mathrm{s}}$. Given the particular form of the framing dependence, the resulting expressions will also be series expansions in $p_{\alpha}$. The expectation values in canonical framing $\left\langle W_{R_{\alpha}}(\mathcal{L})\right\rangle_{(0, \ldots, 0)}$ can be written as rational functions of the variables $y=\mathrm{e}^{\mathrm{i} \lambda / 2}, x=\mathrm{e}^{\mathrm{i} g_{\mathrm{s}} / 2}$. Therefore, the expansion is straightforward, although somewhat tedious. Without giving more details here, let us record the final answer for this computation. We will write down only the genus zero contribution, i.e., the coefficient of $g_{\mathrm{s}}^{-2}$ in the final expression for the free energy, 
truncated to terms up to degree three ${ }^{5}$

$$
\begin{aligned}
\mathcal{F}_{\mathrm{op}}^{(0)} & \left(g_{\mathrm{s}}, q_{1}^{\prime}, q_{2}^{\prime}, q_{c}, \lambda_{1}, \lambda_{2}\right) \\
= & y_{1}^{2}+\frac{1}{8} y_{1}^{4}+\frac{1}{27} y_{1}^{6}+y_{2}^{2}+\frac{1}{8} y_{2}^{4}+\frac{1}{27} y_{2}^{6} \\
& +q_{1}^{\prime}\left(y_{1}-y_{1}^{-1}\right)+q_{2}^{\prime}\left(y_{2}-y_{2}^{-1}\right)+q_{c}\left(y_{1} y_{2}-y_{1} y_{2}^{-1}-y_{1}^{-1} y_{2}-y_{1}^{-1} y_{2}^{-1}\right) \\
& -\frac{1}{8} q_{1}^{\prime 2}\left(7 y_{1}^{2}-8+y_{1}^{-2}\right)-\frac{1}{8} q_{2}^{\prime 2}\left(7 y_{2}^{2}-8+y_{2}^{-2}\right) \\
& -q_{1}^{\prime} q_{2}^{\prime}\left(y_{1} y_{2}-y_{1} y_{2}^{-1}-y_{1}^{-1} y_{2}+y_{1}^{-1} y_{2}^{-1}\right) \\
& -\frac{1}{8} q_{c}^{2}\left(7 y_{1}^{2} y_{2}^{2}-8 y_{1}^{2}-8 y_{2}^{2}+8+y_{1}^{2} y_{2}^{-2}+y_{1}^{-2} y_{2}^{2}-y_{1}^{-2} y_{2}^{-2}\right) \\
& -q_{1}^{\prime} q_{c}\left(y_{1}^{2} y_{2}-y_{2}-y_{1}^{2} y_{2}^{-1}+y_{1}^{-1}\right)-q_{2}^{\prime} q_{c}\left(y_{1} y_{2}^{2}-y_{1}-y_{1}^{-1} y_{2}^{2}+y_{1}^{-1}\right) \\
& +\frac{1}{27} q_{1}^{\prime 3}\left(55 y_{1}^{3}-81 y_{1}+27 y_{1}^{-1}-y_{1}^{-3}\right) \\
& +\frac{1}{27} q_{2}^{\prime 3}\left(55 y_{2}^{3}-81 y_{2}+27 y_{2}^{-1}-y_{2}^{-3}\right) \\
& +q_{1}^{\prime 2} q_{2}^{\prime}\left(3 y_{1}^{2} y_{2}-4 y_{2}-3 y_{1}^{2} y_{2}^{-1}+4 y_{2}^{-1}+y_{1}^{-2} y_{2}-y_{1}^{-2} y_{2}^{-1}\right) \\
& +q_{1}^{\prime} q_{2}^{\prime 2}\left(3 y_{1} y_{2}^{2}-4 y_{1}-3 y_{1}^{-1} y_{2}^{2}+4 y_{1}^{-1}+y_{1} y_{2}^{-2}-y_{1}^{-1} y_{2}^{-2}\right) \\
& +q_{1}^{\prime 2} q_{c}\left(3 y_{1}^{3} y_{2}-4 y_{1} y_{2}-3 y_{1}^{3} y_{2}^{-1}+4 y_{1} y_{2}^{-1}+y_{1}^{-1} y_{2}-y_{1}^{-1} y_{2}^{-1}\right) \\
& +q_{1}^{\prime} q_{c}^{2}\left(3 y_{1}^{3} y_{2}^{2}-4 y_{1}^{3}-4 y_{1} y_{2}^{2}+5 y_{1}+y_{1}^{-1} y_{2}^{2}+y_{1}^{3} y_{2}^{-2}-y_{1} y_{2}^{-2}-y_{1}^{-1}\right) \\
& +q_{2}^{\prime 2} q_{c}\left(3 y_{1} y_{2}^{3}-4 y_{1} y_{2}-3 y_{1}^{-1} y_{2}^{3}+4 y_{1}^{-1} y_{2}+y_{1} y_{2}^{-1}-y_{1}^{-1} y_{2}^{-1}\right) \\
& +q_{2}^{\prime} q_{c}^{2}\left(3 y_{1}^{2} y_{2}^{3}-4 y_{2}^{3}-4 y_{1}^{2} y_{2}+5 y_{2}+y_{1}^{2} y_{2}^{-1}+y_{1}^{-2} y_{2}^{3}-y_{1}^{-2} y_{2}-y_{2}^{-1}\right) \\
& +q_{1}^{\prime} q_{2}^{\prime} q_{c}\left(3 y_{1}^{2} y_{2}^{2}-4 y_{1}^{2}-4 y_{2}^{2}+5+y_{1}^{2} y_{2}^{-2}+y_{1}^{-2} y_{2}^{2}-y_{1}^{-2}-y_{2}^{-2}\right) \\
& +q_{c}^{3}\left(2 y_{1}^{3} y_{2}^{3}-3 y_{1}^{3} y_{2}-3 y_{1} y_{2}^{3}+4 y_{1} y_{2}+y_{1}^{3} y_{2}^{-1}\right. \\
& \left.+y_{1}^{-1} y_{2}^{3}-y_{1} y_{2}^{-1}-y_{1}^{-1} y_{2}\right) \\
&
\end{aligned}
$$

A first observation is that the final answer does not depend on $z$, as promised above. Moreover, it is not hard to show that the above formula has the correct integrality properties of a genus zero closed string expansion. In order to compare with the Gromov-Witten expansion of the local $d P_{2}$ model, we have to rewrite equation (5.25) in terms of closed string variables using

\footnotetext{
${ }^{5}$ There is a subtlety here. The instanton sum (5.20) contains only terms of genus zero. It is not a priori clear that higher genus corrections do not affect the final answer for the genus zero free energy. In principle, this could happen since the Chern-Simons expansion generates various powers of $g_{\mathrm{s}}$, which mix with the instanton corrections in a nontrivial way. However, in all known examples of generic transitions (present cases included), it is a posteriori clear that such effects are absent. This pattern will be confirmed for compact hypersurfaces in Section 7.
} 
the duality map

$$
y_{1}^{2}=\tilde{q}_{1}, \quad y_{2}^{2}=\tilde{q}_{2}, \quad q_{1}^{\prime} y_{1}=q_{2}^{\prime} y_{2}=q_{c} y_{1} y_{2}=\tilde{q} .
$$

Here $\tilde{q}_{1}, \tilde{q}_{2}$ and $\tilde{q}$ are the instanton factors associated to curve classes $e_{1}, e_{2}$ and $h$ on $d P_{2}$. Then we obtain the following expression

$$
\begin{aligned}
\mathcal{F}_{\mathrm{cl}}^{(0)}\left(\tilde{q}_{1}, \tilde{q}_{2}, \tilde{q}\right)= & \tilde{q}_{1}+\tilde{q}_{2}+\tilde{q}\left(\tilde{q}_{1}^{-1} \tilde{q}_{2}^{-1}-2 \tilde{q}_{1}^{-1}-2 \tilde{q}_{2}^{-1}+3\right) \\
& +\tilde{q}^{2}\left(-4 \tilde{q}_{1}^{-1} \tilde{q}_{2}^{-1}+5 \tilde{q}_{1}^{-1}+5 \tilde{q}_{2}^{-1}-6\right) \\
& +\tilde{q}^{3}\left(-6 \tilde{q}_{1}^{-2} \tilde{q}_{2}^{-1}-6 \tilde{q}_{1}^{-1} \tilde{q}_{2}^{-2}+7 \tilde{q}_{1}^{-2}+7 \tilde{q}_{2}^{-2}+35 \tilde{q}_{1}^{-1} \tilde{q}_{2}^{-1}\right. \\
& \left.-32 \tilde{q}_{1}^{-1}-32 \tilde{q}_{2}^{-1}+27\right)+\cdots \\
& +\frac{1}{4}\left[\tilde{q}_{1}^{2}+\tilde{q}_{2}^{2}+\tilde{q}^{2}\left(\tilde{q}_{1}^{-2} \tilde{q}_{2}^{-2}-2 \tilde{q}_{1}^{-2}-2 \tilde{q}_{2}^{-2}+3\right)+\cdots\right] \\
& +\frac{1}{27}\left[\tilde{q}_{1}^{3}+\tilde{q}_{2}^{3}+\tilde{q}^{3}\left(\tilde{q}_{1}^{-3} \tilde{q}_{2}^{-3}-2 \tilde{q}_{1}^{-3}-2 \tilde{q}_{2}^{-3}+3\right)+\cdots\right]+\cdots,
\end{aligned}
$$

which is precisely the genus zero Gromov-Witten expansion of $d P_{2}$.

This result has been obtained previously in ref. [16]. The novelty here is that we have a much better understanding of the choices made in the process. In particular, we have found and tested a general framework for these computations which does not require fixing the toric weights. This formalism is closer in spirit to closed string localization computations on moduli spaces of stable maps. Similar techniques can be applied at higher genus, although the localization computations for arbitrary $z$ become more involved. We will not try to pursue these computations here, but there is little doubt that the results will be in perfect agreement with the closed string expansion.

If one is interested in computational power, a particular choice of $z$ may be very useful. For example, we could choose $z=0$ as in ref. [16], in which case we would obtain a closed form for the instanton corrections

$$
\begin{aligned}
F_{\text {inst }}= & \sum_{d=1}^{\infty} \frac{\mathrm{i} q_{1}^{\prime d}}{2 d \sin \left(d g_{\mathrm{s}} / 2\right)} \operatorname{Tr} V_{1}^{\prime d}+\frac{\mathrm{i} q_{2}^{\prime d}}{2 d \sin \left(d g_{\mathrm{s}} / 2\right)} \operatorname{Tr} V_{2}^{\prime d} \\
& +\sum_{d=1}^{\infty} \frac{q_{1}^{\prime} q_{2}^{\prime}}{d} \operatorname{Tr} V_{1}^{\prime d} \operatorname{Tr} V_{2}^{\prime d}+\sum_{d=1}^{\infty} \frac{(-1)^{d} q_{c}^{d}}{d} \operatorname{Tr} U_{1}^{\prime d} \operatorname{Tr} U_{2}^{\prime d} .
\end{aligned}
$$

The framings of the four knots can be read off from equation (5.19) as $p_{1}=p_{2}=2, q_{1}=q_{2}=\frac{1}{2}$. Therefore, we still have fractional framing for the knots $\Xi_{1}^{\prime}$ and $\Xi_{2}^{\prime}$. Using this expression, one can perform the Chern-Simons integration as in ref. [16] obtaining agreement with the closed string dual for all genera up to degree 4. 
It is interesting to note that this system of Chern-Simons theories is different from the one found in ref. [16], although they give rise to identical expressions for the free energy. This is a very interesting phenomenon whose origin can be traced to a special symmetry of the Gromov-Witten expansion of the local $d P_{2}$ model. We will sketch an argument here, leaving a more thorough investigation of this aspect for future work. Let us recall the expression for the instanton corrections found in ref. [16]

$$
\begin{aligned}
F_{\text {inst }}= & \sum_{d=1}^{\infty} \frac{\mathrm{i} q_{1}^{\prime d}}{2 d \sin \left(d g_{\mathrm{s}} / 2\right)} \operatorname{Tr} V_{1}^{\prime d}+\frac{\mathrm{i} q_{2}^{\prime d}}{2 d \sin \left(d g_{\mathrm{s}} / 2\right)} \operatorname{Tr} V_{2}^{\prime d} \\
& +2 \sum_{d=1}^{\infty} \frac{q_{1}^{\prime} q_{2}^{\prime}}{d} \operatorname{Tr} V_{1}^{\prime d} \operatorname{Tr} V_{2}^{\prime d}-\sum_{d=1}^{\infty} \frac{q_{c}^{d}}{d} \operatorname{Tr} U_{1}^{\prime d} \operatorname{Tr} U_{2}^{\prime d}
\end{aligned}
$$

with framings $p_{1}=p_{2}=2$ and $q_{1}=q_{2}=0$. Note that the contributions of multicovers of the annulus $A$ and the pinched cylinder $D_{1}^{\prime} \cap D_{2}^{\prime}$ have different coefficients, and also $q_{1}$ and $q_{2}$ have different values.

In order to understand the meaning of these different choices, it suffices to consider the terms of order 2 in the free energy. More precisely, let us look at the degree 2 multicover contributions of the annulus $A$. Formula (5.28) yields

$$
\frac{1}{8} q_{c}^{2}\left(y_{1}^{2} y_{2}^{2}-y_{1}^{2} y_{2}^{-2}-y_{1}^{-2} y_{2}^{2}-y_{1}^{-2} y_{2}^{-2}\right)-q_{c}^{2}\left(y_{1}^{2} y_{2}^{2}-y_{1}^{2}-y_{2}^{2}+1\right) .
$$

The first term in this formula represents degree 2 multicovers of the degree 1 contribution. The second term represents a nontrivial contribution to the degree 2 Gromov-Witten invariants. If we perform a similar computation starting with equation (5.29), we find that only the multicover contributions are present. Hence in this case, the degree 2 annulus corrections do not generate genuine new contributions to the Gromov-Witten invariants of degree 2. Instead, the corrections corresponding to the pinched cylinder $D_{1}^{\prime} \cap D_{2}^{\prime}$ have to be counted twice in order to compensate for the missing terms. Of course, it is highly nontrivial that this correction yields the correct results at higher degree as well.

From the closed string point of view, this phenomenon has the following interpretation. Let us consider, e.g., the localization computations for the Gromov-Witten invariant of the local $d P_{2}$ model in curve class $2 h-e_{1}-e_{2}$. Adopting a Kontsevich graph representation [36] of the fixed loci, we obtain the graphs in figure 3.

These graphs can be easily evaluated according to standard deformation theory techniques. For $z=0$, one finds that graphs I, II and III contribute -1 each while the sum of the graphs IV, V and VI is also -1 . The total 


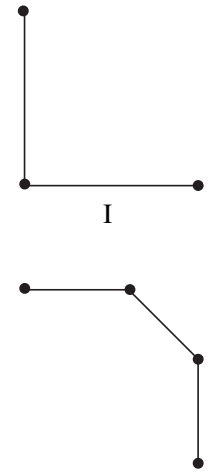

IV
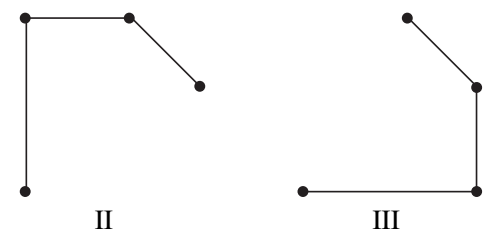

III

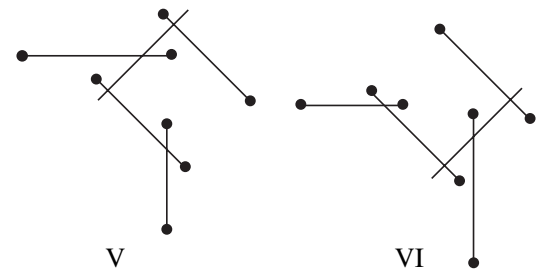

Figure 3: Closed string Kontsevich graphs in the class $2 h-e_{1}-e_{2}$.

is -4 as expected. Now, we can establish a correspondence between these contributions and various terms of degree 2 in the open string free energy (equation (5.25)). The contributions of I, II and III correspond to the terms $-q_{1}^{\prime} q_{2}^{\prime} y_{1}^{-1} y_{2}^{-1},-q_{1}^{\prime} q_{c} y_{1}^{-1}$ and, respectively, $-q_{2}^{\prime} q_{c} y_{2}^{-1}$. The first term arises in the Chern-Simons evaluation of the correction $q_{1}^{\prime} q_{2}^{\prime} \operatorname{Tr} V_{1} \operatorname{Tr} V_{2}$ associated to the pinched cylinder. The next two terms appear in the Chern-Simons evaluation of the connected expectation values

$$
\begin{aligned}
& (-\mathrm{i}) q_{1}^{\prime} q_{c}\left[\left\langle\operatorname{Tr} V_{1}^{\prime} \operatorname{Tr} U_{1}^{\prime}\right\rangle-\left\langle\operatorname{Tr} V_{1}^{\prime}\right\rangle\left\langle\operatorname{Tr} U_{1}^{\prime}\right\rangle\right]\left\langle\operatorname{Tr} U_{2}^{\prime}\right\rangle \\
& (-\mathrm{i}) q_{2}^{\prime} q_{c}\left[\left\langle\operatorname{Tr} V_{2}^{\prime} \operatorname{Tr} U_{2}^{\prime}\right\rangle-\left\langle\operatorname{Tr} V_{2}^{\prime}\right\rangle\left\langle\operatorname{Tr} U_{2}^{\prime}\right\rangle\right]\left\langle\operatorname{Tr} U_{1}^{\prime}\right\rangle
\end{aligned}
$$

of the links $\left(\Gamma_{1}^{\prime}, \Xi_{1}^{\prime}\right)$, and, respectively, $\left(\Gamma_{2}^{\prime}, \Xi_{2}^{\prime}\right)$. Note that there is a clear geometric interpretation of this correspondence since each invariant primitive open string instanton corresponds to an invariant primitive closed string instanton. According to this rule, we would expect the sum over the last three graphs to be associated to a term in the Chern-Simons expansion of the following annulus instanton corrections

$$
\frac{q_{c}^{2}}{2}\left[\left\langle\operatorname{Tr} U_{1}^{\prime 2}\right\rangle\left\langle\operatorname{Tr} U_{2}^{\prime 2}\right\rangle+\frac{1}{2}\left\langle\left(\operatorname{Tr} U_{1}^{\prime}\right)^{2}\right\rangle\left\langle\left(\operatorname{Tr} U_{2}^{\prime}\right)^{2}\right\rangle-\frac{1}{2}\left\langle\operatorname{Tr} U_{1}^{\prime}\right\rangle^{2}\left\langle\operatorname{Tr} U_{2}^{\prime}\right\rangle^{2}\right] .
$$

This is indeed true if we use the instanton series (5.28). The relevant term is $-q_{c}^{2}$ in equation (5.30). However, if we use equation (5.29) there is no such term in the expression of the free energy. Instead, we find that the term $-q_{1}^{\prime} q_{2}^{\prime} y_{1}^{-1} y_{2}^{-1}$ appears with coefficient 2 , so that the final answer in closed string variables is the same. Moreover, similar phenomena can be noticed for higher degree terms in the expansion. This suggests a nontrivial symmetry in the graph representation of Gromov-Witten invariants of the local $d P_{2}$ model. Perhaps this symmetry can be better understood if we regard the 


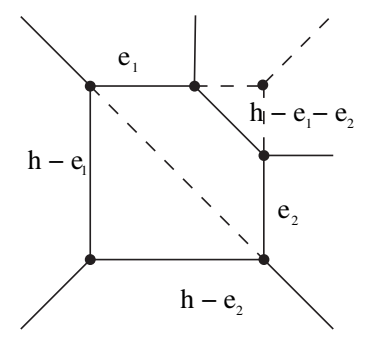

Figure 4: $F_{0}$ and $d P_{2}$ discriminant loci.

$d P_{2}$ as the one-point blow-up of a Hirzebruch $\mathbb{F}_{0}$ surface. Obviously, the Kontsevich graphs of the local $\mathbb{F}_{0}$ model are symmetric with respect to reflections with respect to the diagonal (see figure 4).

The above observation suggests that this symmetry is somehow present in a hidden form in the graph expansion of the local $d P_{2}$ model, at least for a specific choice of weights. These issues deserve a more detailed investigation since they are very likely related to localization of the integral invariants of Gopakumar and Vafa.

To summarize this discussion, we conclude that both systems of ChernSimons theories represent sensible large $N$ dual models for the local $d P_{2}$ geometry. However, the model specified by equation (5.28) is preferable from a conceptual point of view since it was obtained as a result of a rather general set of rules. Essentially, the only ambiguities left in this construction are related to the sign of various open string corrections, which cannot be fixed rigorously at the present stage. To illustrate the general character of this approach, let us briefly consider other local transitions.

\subsection{Local $d P_{3}$ and $d P_{5}$ transitions}

The remaining local models can be analyzed along the same lines. Given the deformed hypersurface equations

$$
\begin{array}{ll}
d P_{3}: & U Z_{1} Z_{4}+V Z_{2} Z_{3}=\mu, \\
d P_{5}: & U Z_{1} Z_{2}+V Z_{3} Z_{4}=\mu,
\end{array}
$$

we can find again the vanishing cycles for each model. For local $d P_{3}$, we find two spheres $L_{1}$ and $L_{2}$ cut by the following real equations on $X_{\mu}$

$$
\begin{aligned}
L_{1}: & U Z_{2} \bar{Z}_{2} Z_{4}=\bar{Z}_{1}, & V Z_{2} Z_{4} \bar{Z}_{4}=\bar{Z}_{3}, \\
L_{2}: & U Z_{1} \bar{Z}_{1} Z_{3}=\bar{Z}_{2}, & V Z_{1} Z_{3} \bar{Z}_{3}=\bar{Z}_{4} .
\end{aligned}
$$


In terms of local coordinates $\left(x_{1}, y_{1}, u_{1}, v_{1}\right),\left(x_{2}, y_{2}, u_{2}, v_{2}\right)$ centered around the points $P_{1}, P_{2}$, the equations of $L_{1}, L_{2}$ take the canonical form

$$
\begin{aligned}
& L_{1}: \quad x_{1} u_{1}+y_{1} v_{1}=\mu, \quad u_{1}=\bar{x}_{1}, \quad v_{1}=\bar{y}_{1}, \\
& L_{2}: \quad x_{2} u_{2}+y_{2} v_{2}=\mu, \quad u_{2}=\bar{x}_{2}, \quad v_{2}=\bar{y}_{2} \text {. }
\end{aligned}
$$

For local $d P_{5}$, we obtain similarly four spheres $L_{1}, L_{2}, L_{3}, L_{4}$, whose local equations are identical to (5.35) when written in terms of coordinates centered at $P_{1}, P_{2}, P_{3}, P_{4}$. The resulting geometry is represented in figure 5 below.

In order to proceed with the localization computations, we have to define suitable torus actions on the deformed hypersurfaces $X_{\mu}$, which preserve the vanishing cycles. It turns out that we can choose the same form of the action for both local models

$$
\begin{array}{ccccccc} 
& Z_{1} & Z_{2} & Z_{3} & Z_{4} & U & V \\
S^{1} & \lambda_{1} & 0 & \lambda_{3} & 0 & -\lambda_{1} & -\lambda_{3} .
\end{array}
$$

The next step is to identify the invariant open string Riemann surfaces in $X_{\mu}$ with boundary components contained in the 3 -spheres. This can be done using the same techniques as in the previous section. Namely, we consider projective completions $\bar{X}_{\mu}$, and identify all $S^{1}$-invariant curves thereof which intersect the vanishing cycles. This results in a collection of invariant discs and annuli embedded in $\bar{X}_{\mu}$. For the local transitions we have to keep only those surfaces which do not have points at infinity, as before. We will not repeat all the details of this algorithm, since it is very similar to the local $d P_{2}$ example. Moreover, we will study the compact version of the $d P_{3}$ model in great detail in the next section. The resulting configurations are represented below.

Note that in the $d P_{3}$ case, the open string maps localize on a collection of four discs $D_{3}^{\prime}, D_{4}^{\prime}, D_{3}^{\prime \prime}$ and $D_{4}^{\prime \prime}$ so that $D_{3}^{\prime}$ and $D_{4}^{\prime \prime}$ end on $L_{1}$, whereas $D_{3}^{\prime \prime}$ and $D_{4}^{\prime}$ end on $L_{2}$. $D_{3}^{\prime}$ and $D_{4}^{\prime}$ have common origin $Q_{3}=\left\{Z_{1}=Z_{4}=U=0\right.$, $\left.V Z_{2} Z_{3}=\mu\right\}$ and $D_{3}^{\prime \prime}$ and $D_{4}^{\prime \prime}$ have common origin $Q_{4}=\left\{Z_{2}=Z_{3}=V=0\right.$, $\left.U Z_{1} Z_{4}=\mu\right\}$. We will denote the boundary components of $D_{3}^{\prime}, D_{4}^{\prime}, D_{3}^{\prime \prime}$ and $D_{4}^{\prime \prime}$ by $\Gamma_{3}^{\prime}, \Gamma_{4}^{\prime}, \Gamma_{3}^{\prime \prime}$ and $\Gamma_{4}^{\prime \prime}$, respectively. A short local computation shows that $\left(\Gamma_{3}^{\prime}, \Gamma_{4}^{\prime \prime}\right)$ and, respectively, $\left(\Gamma_{3}^{\prime \prime}, \Gamma_{4}^{\prime}\right)$ form algebraic Hopf links in $L_{1}$ and $L_{2}$ with linking number $l=+1$. We will also denote the holonomy variables associated to the four link components by $V_{3}^{\prime}, V_{4}^{\prime}, V_{3}^{\prime \prime}$ and $V_{4}^{\prime \prime}$. In the $d P_{5}$ example, the open string maps localize on a collection of four annuli $A_{13}, A_{14}, A_{23}$ and $A_{24}$. The boundary components of each pair of annuli ending on a given sphere form again an algebraic Hopf link with linking number +1 . Let us denote by $V_{1}^{\prime}, \ldots, V_{4}^{\prime \prime}$ the holonomy variables associated to the eight boundary components, as shown in figure 5 . 

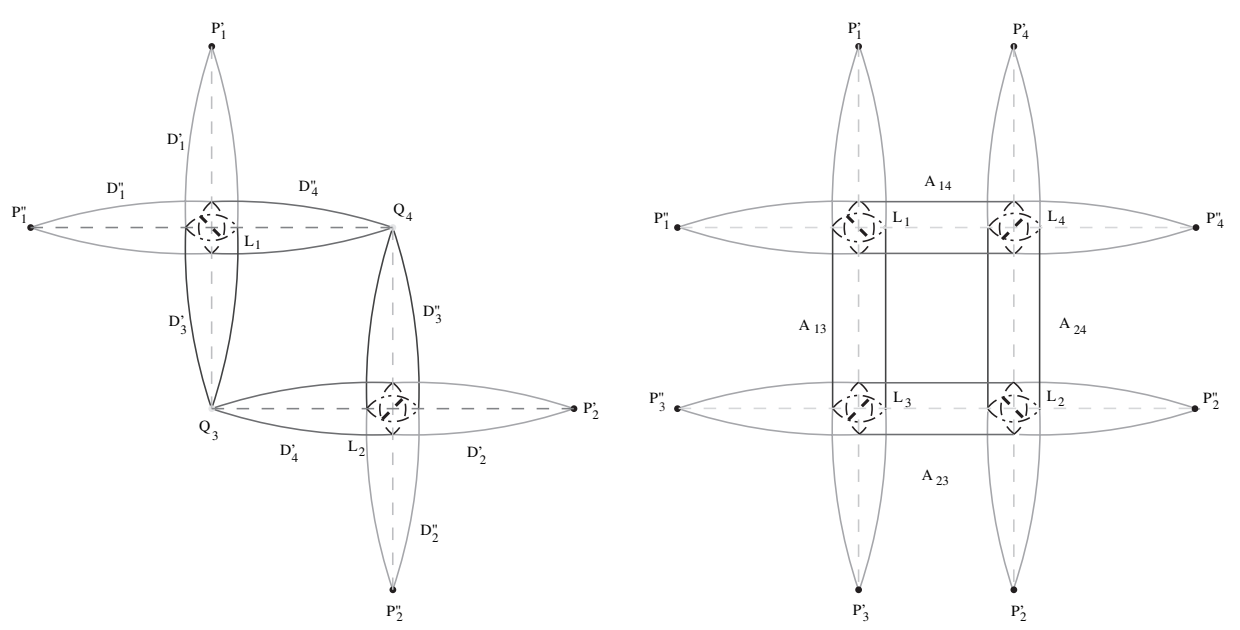

Figure 5: Primitive open string instantons on $X$.

Next, we have to compute the instanton corrections $F_{\text {inst }}$ for these configurations. To keep the exposition simple, for the $d P_{3}$ model we will only write down genus zero corrections up to degree 2 . For the $d P_{5}$ model, we can write down a closed form expression for all instanton corrections. The results of localization computations can be written as rational functions of $z=\lambda_{3} / \lambda_{1}$. We find the following expansions.

Local $d P_{3}$ :

$$
\begin{aligned}
F_{\text {inst }}= & -\frac{\mathrm{i} q_{3}^{\prime}}{g_{\mathrm{s}}} \operatorname{Tr} V_{3}^{\prime}-\frac{\mathrm{i} q_{3}^{\prime \prime}}{g_{\mathrm{s}}} \operatorname{Tr} V_{3}^{\prime \prime}-\frac{\mathrm{i} q_{4}^{\prime}}{g_{\mathrm{s}}} \operatorname{Tr} V_{4}^{\prime}-\frac{\mathrm{i} q_{4}^{\prime \prime}}{g_{\mathrm{s}}} \operatorname{Tr} V_{4}^{\prime \prime}+\frac{\mathrm{i} q_{3}^{\prime 2}}{g_{\mathrm{s}}} \frac{2-z}{4 z} \operatorname{Tr} V_{3}^{\prime 2} \\
& +\frac{\mathrm{i} q_{3}^{\prime 2}}{g_{\mathrm{s}}} \frac{2-z}{4 z} \operatorname{Tr} V_{3}^{\prime \prime 2} \\
& +\frac{\mathrm{i} q_{4}^{\prime 2}}{q_{\mathrm{s}}} \frac{2 z-1}{4} \operatorname{Tr} V_{4}^{\prime 2}+\frac{\mathrm{i} q_{4}^{\prime \prime 2}}{q_{\mathrm{s}}} \frac{2 z-1}{4} \operatorname{Tr} V_{4}^{\prime \prime 2} \\
& -q_{3}^{\prime 2} \frac{z-1}{4 z^{2}}\left(\operatorname{Tr} V_{3}^{\prime}\right)^{2}-q_{3}^{\prime \prime 2} \frac{z-1}{4 z^{2}}\left(\operatorname{Tr} V_{3}^{\prime \prime}\right)^{2} \\
& -q_{4}^{\prime 2} \frac{z(1-z)}{4}\left(\operatorname{Tr} V_{4}^{\prime}\right)^{2}-q_{4}^{\prime 2} \frac{z(1-z)}{4}\left(\operatorname{Tr} V_{4}^{\prime \prime}\right)^{2} \\
& +q_{3}^{\prime} q_{4}^{\prime} \operatorname{Tr} V_{3}^{\prime} \operatorname{Tr} V_{4}^{\prime}+q_{3}^{\prime \prime} q_{4}^{\prime \prime} \operatorname{Tr} V_{3}^{\prime \prime} \operatorname{Tr} V_{4}^{\prime \prime}
\end{aligned}
$$

where $q_{3}^{\prime}, q_{3}^{\prime \prime}, q_{4}^{\prime}$ and $q_{4}^{\prime \prime}$ are open string Kähler moduli associated to the four discs. 
Local $d P_{5}$ :

$$
\begin{aligned}
F_{\text {inst }}= & \sum_{n=1}^{\infty}\left(\frac{q_{13}^{n}}{n} \operatorname{Tr} V_{1}^{\prime n} \operatorname{Tr} V_{3}^{\prime n}+\frac{q_{14}^{n}}{n} \operatorname{Tr} V_{1}^{\prime \prime n} \operatorname{Tr} V_{4}^{\prime \prime n}\right. \\
& \left.+\frac{q_{23}^{n}}{n} \operatorname{Tr} V_{2}^{\prime \prime n} \operatorname{Tr} V_{3}^{\prime \prime n}+\frac{q_{24}^{n}}{n} \operatorname{Tr} V_{2}^{\prime n} \operatorname{Tr} V_{4}^{\prime n}\right)
\end{aligned}
$$

where $q_{13}, q_{14}, q_{23}$ and $q_{24}$ are open string Kähler moduli associated to the four cylinders. Note that in both expressions we made particular choices of signs for the open string computations. For the $d P_{3}$ model, the correct choice involves an alternating sign in the multicover contributions of a single disc. Since all open string invariant maps can be reduced to discs and closed string components using a normalization sequence, this choice fixes the sign ambiguity for all localization contributions. Note also that the choice made for the annulus corrections in the $d P_{5}$ example is different from the corresponding choice in the $d P_{2}$ case. We will discuss a potential explanation for this discrepancy after fixing the framing, which is the last piece of the puzzle.

In order to determine the framing for all boundary components, we will proceed by analogy with local $d P_{2}$ example. Namely, we will impose $S^{1}$-invariance for all sections to the normal bundles to the knots. After a straightforward computation, we find the following values

$$
\begin{array}{rlrl}
d P_{3}: & p_{3}^{\prime}=p_{3}^{\prime \prime}=\frac{1}{z}, & p_{4}^{\prime}=p_{4}^{\prime \prime}=z \\
d P_{5}: & p_{1}^{\prime}=p_{2}^{\prime}=\frac{1}{z}, & p_{3}^{\prime}=p_{4}^{\prime}=-\frac{1}{z}, \\
p_{1}^{\prime \prime}=p_{2}^{\prime \prime}=z, & p_{3}^{\prime \prime}=p_{4}^{\prime \prime}=-z .
\end{array}
$$

As discussed before, the framings are to be thought of as formal variables. In order to perform the Chern-Simons functional integral, we have to expand all expectation values of knots and links as formal power series in $g_{\mathrm{s}}$. Following the same steps as in the $d P_{2}$ example, one can show that the resulting free energy agrees with the closed string expansions of the local $d P_{3}$ and $d P_{5}$ models. ${ }^{6}$ In particular, the final answer is independent of $z$, without making any further choices.

Several remarks are in order here. The $d P_{5}$ example has been considered before in ref. [5]. The dual Chern-Simons theory found there is

\footnotetext{
${ }^{6}$ There are some subtleties with the closed string instanton expansion for $d P_{5}$ related to the fact that the toric model is not generic.
} 
formally identical to equation (5.38), except that the framing is integervalued. However, it was also shown there that for annulus corrections, the result depends only on the effective framing, which is defined as the sum of the framing assigned to the two boundary components. In our case, we have to treat the framing as a formal variable, but the conclusion turns out to be the same, i.e., the final result depends only on the effective framing, which is an integer in all cases. For the annulus in the $d P_{2}$ model, the effective framing is $q_{1}+q_{2}=1$, while for all annuli in the $d P_{5}$ model, the effective framing is zero. These values are in agreement with the general prescription given in ref. [5], except that our algorithm is different and it applies equally well to invariant discs. In the later case, treating the framing as a formal variable is crucial since the corrections themselves have a similar dependence.

The effective framing of a cylinder has a simple geometric dependence, which can be described as follows. Let us note that each invariant open string surface on $X_{\mu}$ corresponds to an invariant rational curve on the resolution $\widetilde{X}$. Using this correspondence, we can find a correlation between the effective framing of a cylinder and the normal bundle of the associated curve on $\widetilde{X}$. Suppose an annulus $A$ corresponds to a curve $C$, and let $S \subset X$ denote the image of the zero section. The normal bundle $N_{C / X}$ is an extension of $i_{C}^{*} N_{S / X}$ by $N_{C / S}$ which is typically split. It can be easily seen that the effective framing of $A$ is given by $\operatorname{deg}\left(i_{C}^{*} N_{S / X}\right)$. Presumably, this rule is consistent with the general prescription of ref. [5], which is formulated in a different language.

Finally, there is one more aspect that deserves a few comments. We noted above that the sign ambiguity was given different resolutions in the $d P_{2}$ and, respectively, $d P_{5}$ models. Note that a concise rule encompassing all choices is to take the sign of a term of degree $n$ to be $(-1)^{n p}$ where $p$ is the effective framing of the cylinder. This rule is very likely connected to a subtle sign problem found in a similar context in ref. [47]. There it was found that the holonomy variable for a knot had to be redefined by a sign $(-1)^{p}$ in order to obtain the desired integrality properties. We believe that these problems are related, but we will not discuss this aspect further here.

In conclusion, the main idea developed in this section is that the proper framework for open string amplitudes is a formal extension of Chern-Simons theory which allows the framing to become fractional. Within this formalism, we have found a natural relation between framing and the torus weights which can be uniformly applied to local transitions. In the next sections, we will extend the analysis of this section to transitions between compact Calabi-Yau manifolds. 


\section{Localization of open string morphisms to compact three-folds}

After this rather long digression, we return to open string amplitudes on compact Calabi-Yau target spaces. We outlined the main principles of the approach in Section 3. In this section, we will carry out this program in detail for one of the models introduced in Section 4. For concreteness, we focus on the compact transition based on the local $d P_{3}$ model. The other models admit a similar treatment.

To fix ideas, let us recall the toric data of the ambient toric variety $\mathcal{Z}$

$\begin{array}{lccccccc} & Z_{1} & Z_{2} & Z_{3} & Z_{4} & U & V & W \\ \mathbb{C}^{*} & 1 & 1 & 0 & 0 & -1 & -1 & 0 \\ \mathbb{C}^{*} & 0 & 0 & 1 & 1 & -1 & -1 & 0 \\ \mathbb{C}^{*} & 0 & 0 & 0 & 0 & 1 & 1 & 1\end{array}$

and the generic Calabi-Yau hypersurface

$$
\sum_{a, b, c \geq 0, a+b+c=3} U^{a} V^{b} W^{c} f_{a b c}\left(Z_{i}\right)=0 .
$$

Here $f_{a b c}\left(Z_{i}\right)$ are bihomogeneous polynomials of $\left(Z_{1}, Z_{2}\right)$ and, respectively, $\left(Z_{3}, Z_{4}\right)$ of bidegree $(a+b, a+b)$. We consider a one-parameter family of hypersurfaces $Y_{\mu}$ defined by

$$
\left(U Z_{1} Z_{4}+V Z_{2} Z_{3}-\mu W\right) W^{2}+\sum_{(a, b, c)^{\prime}} U^{a} V^{b} W^{c} f_{a b c}\left(Z_{i}\right)=0
$$

where the triples $(a, b, c)^{\prime}$ take all allowed values except $(1,0,2),(0,1,2)$, $(0,0,3)$. The coefficients of $f_{a b c}\left(Z_{i}\right)$ are fixed to some generic values. One can check that equation (6.3) is smooth for generic $\mu \neq 0$ and develops two ordinary double points

$$
P_{1}: \quad Z_{1}=Z_{3}=U=V=0, \quad P_{2}: \quad Z_{2}=Z_{4}=U=V=0
$$

at $\mu=0$. As discussed in Section 4 , the two conifold singularities can be simultaneously resolved by performing a toric blow-up of $Z$ along the section $U=V=0$. The strict transform $\widetilde{Y}$ is a crepant resolution of $Y_{0}$. The exceptional locus consists of two homologous curves $C_{1}$ and $C_{2}$.

The smooth fiber $Y_{\mu}, \mu \neq 0$ contains two vanishing cycles $L_{1}$ and $L_{2}$ in the same homology class $\left[L_{1}\right]=\left[L_{2}\right] \in H_{3}\left(Y_{\mu}, \mathbb{Z}\right)$. The topological open string theory considered in this section is defined by wrapping $N_{1}$ and $N_{2} \mathbf{A}$-branes on $L_{1}$ and $L_{2}$. Then the charge constraint (2.4) yields $N_{1}+N_{2}=0$. This means the D-brane configuration should be interpreted as a brane/antibrane system. We have $N=N_{1}$ branes on $L_{1}$ and $N$ anti-branes on $L_{2}$, 
which can be thought of as $N$ branes wrapping the cycle $L_{2}$ with opposite orientation. This is a particular case of our general discussion in which $v=1$ and $r=1$, hence the lattice of vanishing cycles has rank 1. Formula (2.7) for the genus zero topological open string free energy takes the form

$$
\mathcal{F}_{(Y, L) ; \mathrm{op}}^{(0)}\left(g_{\mathrm{s}}, t_{\alpha}, \lambda\right)=\mathcal{F}_{Y ; \mathrm{cl}}^{(0)}\left(g_{\mathrm{s}}, t_{\alpha}\right)+\sum_{\beta \in H_{2}(Z, L)} F_{\beta}^{(0)}\left(g_{\mathrm{s}}, \lambda\right) \mathrm{e}^{-\langle J, \beta\rangle},
$$

where $\lambda=N g_{\mathrm{s}}$ and $t_{\alpha}, \alpha=1,2,3$, are the Kähler moduli of $Y$. In order to compute the coefficients $F_{\beta}^{(0)}\left(g_{\mathrm{s}}, \lambda\right)$, we have to follow the steps outlined in Section 3.

Consider the following $G=\left(S^{1}\right)^{7}$ action on $\mathcal{Z}$

$$
\left(\mathrm{e}^{\mathrm{i} \phi_{1}}, \ldots, \mathrm{e}^{\mathrm{i} \phi_{7}}\right) \cdot\left(Z_{1}, Z_{2}, \ldots, W\right) \longrightarrow\left(\mathrm{e}^{\mathrm{i} \phi_{1}} Z_{1}, \mathrm{e}^{\mathrm{i} \phi_{2}} Z_{2}, \ldots, \mathrm{e}^{\mathrm{i} \phi_{7}} W\right) .
$$

As explained in Section 3, one of the main problems is that the cycles $L_{1}$ and $L_{2}$ are not preserved by the torus action (6.6). The solution is to specialize the triple $\left(Y, L_{1}, L_{2}\right)$ to a degenerate hypersurface $\bar{Y}$ and two cycles $\bar{L}_{1}$ and $\bar{L}_{2}$ which are preserved by a certain subtorus. In general, it is not clear that such a limit in the complex structure moduli space always exists. However, for all the models discussed in Section 4, a suitable degeneration immediately presents itself. Recall that in the local context of the previous section, we had to take a projective completion $\bar{X}_{\mu}$ of $X_{\mu}$, which was a toric Fano three-fold. For the $d P_{3}$ example, the equation of $\bar{X}_{\mu}$ is

$$
U Z_{1} Z_{4}+V Z_{2} Z_{3}=\mu W
$$

Moreover, $\bar{X}_{\mu}$ admits a torus action which preserves the vanishing cycles. The degeneration we are looking for is the reducible nonreduced hypersurface $\bar{Y}$ defined by

$$
\left(U Z_{1} Z_{4}+V Z_{2} Z_{3}-\mu W\right) W^{2}=0 .
$$

$\bar{Y}$ has two components $\bar{Y}_{1}=\bar{X}_{\mu}$ and $\bar{Y}_{2}$ defined by $W^{2}=0$. The cycles $\bar{L}_{1}$ and $\bar{L}_{2}$ are the vanishing cycles defined in equation (5.34), which are obviously embedded in $\bar{Y}$. By construction, the triple $\left(\bar{Y}, \bar{L}_{1}, \bar{L}_{2}\right)$ is preserved by a subtorus $\left(S^{1}\right)^{2} \subset G$. We will give more details on the local geometry below.

Note that similar degenerations can be found without difficulty for the other two models discussed in Section 4. Moreover, we believe that a suitable limit in the complex structure moduli space can be found for all transitions for which the singular points are fixed by the torus action. This is in fact the main restriction on the present approach to open string amplitudes on Calabi-Yau hypersurfaces. 


\subsection{Local geometry on $\mathcal{Z}$}

A thorough analysis of open string maps in the present context requires a systematic description of the local geometry. Recall that the toric four-fold $\mathcal{Z}$ is isomorphic to the projective bundle $\mathbb{P}(\mathcal{O} \oplus \mathcal{O}(-1,-1) \oplus \mathcal{O}(-1,-1))$ over $\mathbb{F}_{0}$, and $U, V$ and $W$ are relative projective coordinates. The torus action (6.6) leaves the sections $S=\{U=V=0\}, S^{\prime}=\{U=W=0\}, S^{\prime \prime}=$ $\{V=W=0\}$ and the fibers $F_{1}=\left\{Z_{1}=Z_{3}=0\right\}, F_{2}=\left\{Z_{2}=Z_{4}=0\right\}, F_{3}=$ $\left\{Z_{1}=Z_{4}=0\right\}, F_{4}=\left\{Z_{2}=Z_{3}=0\right\}$ invariant. Note that $S, S^{\prime}$ and $S^{\prime \prime}$ are isomorphic to $\mathbb{F}_{0}$ and $F_{1}, \ldots, F_{4}$ are isomorphic to $\mathbb{P}^{2}$. The fixed locus of the $G$-action on $\mathcal{Z}$ consists of 12 isolated points $P_{k}, P_{k}^{\prime}, P_{k}^{\prime \prime}, k=1, \ldots, 4$, which are intersection points of $S, S^{\prime}, S^{\prime \prime}$ and $F_{k}$. More precisely, $P_{k}=F_{k} \cap S, P_{k}^{\prime}=$ $F_{k} \cap S^{\prime}, P_{k}^{\prime \prime}=F_{k} \cap S^{\prime \prime}, k=1, \ldots, 4$. For a given $k$, the points $\left(P_{k}, P_{k}^{\prime}, P_{k}^{\prime \prime}\right)$ determine three invariant curves $\overline{P_{k} P_{k}^{\prime}}, \overline{P_{k} P_{k}^{\prime \prime}}$ and $\overline{P_{k}^{\prime} P_{k}^{\prime \prime}}$. The fixed points lying in a given section, say $S$, determine four invariant curves $\overline{P_{1} P_{3}}, \overline{P_{1} P_{4}}$, $\overline{P_{2} P_{3}}$ and $\overline{P_{2} P_{4}}$. The same is true for the sections $S^{\prime}$ and $S^{\prime \prime}$. Overall, we obtain a toric skeleton consisting of 24 invariant curves intersecting at 12 fixed points as in figure 6 .

For later applications, we have introduced a color coding for the homology classes of curves on $\mathcal{Z}$ as follows. We choose a basis in $H_{2}(\mathcal{Z}, \mathbb{Z})$ consisting of the Mori cone generators $\left(h_{1}, h_{2}, h_{3}\right)$. Using equation (A.1) in Appendix A, one can check that $\left(h_{1}, h_{2}, h_{3}\right)$ are represented by the following curves on $\mathcal{Z}$

$$
\begin{array}{ll}
h_{1}: & Z_{1}=Z_{3}=U=0, \\
h_{2}: & Z_{1}=U=V=0, \\
h_{3}: & Z_{3}=U=V=0 .
\end{array}
$$

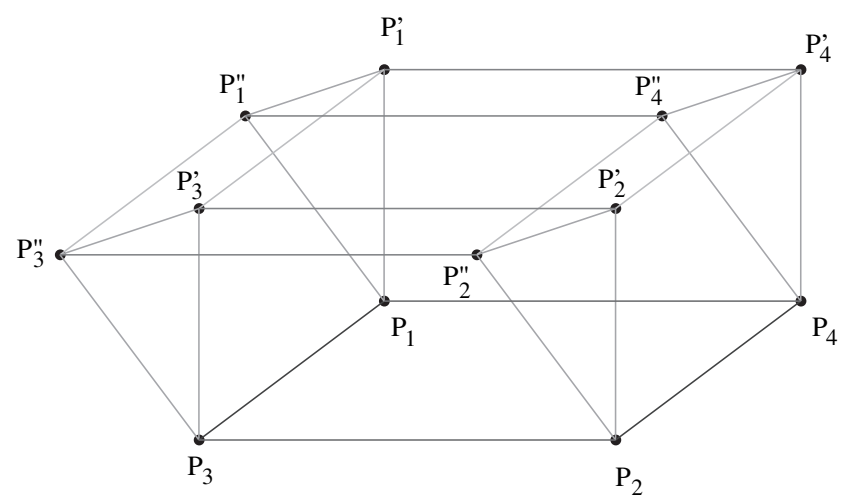

Figure 6: Four-fold skeleton for the generic toric action. The color coding is the following: green $=h_{1}$, blue $=h_{2}$, red $=h_{3}$, cyan $=h_{1}+h_{2}$, magenta $=$ $h_{1}+h_{3}$. 
By construction, $h_{1}$ is the hyperplane class of a $\mathbb{P}^{2}$ fiber of $\mathcal{Z}$ over $\mathbb{F}_{0}$ while $h_{2}$ and $h_{3}$ are $(1,0)$ and, respectively, $(0,1)$ classes of the zero section $U=V=0$. For convenience, we will call $h_{1}$ vertical class, and $h_{2}$ and $h_{3}$ horizontal classes. In figure 6 , green line segments represent curves in the class $h_{1}$, blue line segments represent curves in the class $h_{2}$ and red line segments represent curves in the class $h_{3}$. We also have curves in mixed classes $h_{1}+h_{2}$ represented by cyan line segments and $h_{1}+h_{3}$ represented by magenta line segments. This color coding will also be used later for invariant discs in $\mathcal{Z}$.

This skeleton plays an important part in the localization computation of closed string Gromov-Witten invariants. In the following, we will explain that it also plays an important role in the localization computation of open string instanton corrections.

In order to analyze deformations of open string maps we will also need local coordinates $\left(x_{k}, y_{k}, u_{k}, v_{k}\right),\left(x_{k}^{\prime}, y_{k}^{\prime}, u_{k}^{\prime}, v_{k}^{\prime}\right),\left(x_{k}^{\prime \prime}, y_{k}^{\prime \prime}, u_{k}^{\prime \prime}, v_{k}^{\prime \prime}\right), k=1, \ldots, 4$, centered at each fixed point. We will adopt the following conventions: $\left(x_{k}, y_{k}\right),\left(x_{k}^{\prime}, y_{k}^{\prime}\right)$ and $\left(x_{k}^{\prime \prime}, y_{k}^{\prime \prime}\right)$ are horizontal coordinates along the sections $S$, $S^{\prime}$ and $S^{\prime \prime}$ while $\left(u_{k}, v_{k}\right),\left(u_{k}^{\prime}, v_{k}^{\prime}\right)$ and $\left(u_{k}^{\prime \prime}, v_{k}^{\prime \prime}\right)$ are vertical coordinates along the fibers $F_{k}, k=1, \ldots, 4$. For concreteness, let us write down the affine open subsets and local coordinates centered around $P_{1}, P_{1}^{\prime}$ and $P_{1}^{\prime \prime}$.

$$
\begin{aligned}
& \mathcal{U}_{1}=\left\{Z_{2} \neq 0, Z_{4} \neq 0, W \neq 0\right\}, \\
& \mathcal{U}_{1}^{\prime}=\left\{Z_{2} \neq 0, Z_{4} \neq 0, V \neq 0\right\} \\
& \mathcal{U}_{1}^{\prime \prime}=\left\{Z_{2} \neq 0, Z_{4} \neq 0, U \neq 0\right\} \text {, } \\
& \mathcal{U}_{1}: \quad x_{1}=\frac{Z_{1}}{Z_{2}}, \quad y_{1}=\frac{Z_{3}}{Z_{4}}, \quad u_{1}=\frac{U Z_{2} Z_{4}}{W}, \quad v_{1}=\frac{V Z_{2} Z_{4}}{W}, \\
& \mathcal{U}_{1}^{\prime}: \quad x_{1}^{\prime}=\frac{Z_{1}}{Z_{2}}, \quad y_{1}^{\prime}=\frac{Z_{3}}{Z_{4}}, \quad u_{1}^{\prime}=\frac{U}{V}, \quad v_{1}^{\prime}=\frac{W}{V Z_{2} Z_{4}}, \\
& \mathcal{U}_{1}^{\prime \prime}: \quad x_{1}^{\prime \prime}=\frac{Z_{1}}{Z_{2}}, \quad y_{1}^{\prime \prime}=\frac{Z_{3}}{Z_{4}}, \quad u_{1}^{\prime \prime}=\frac{V}{U}, \quad v_{1}^{\prime \prime}=\frac{W}{U Z_{2} Z_{4}} .
\end{aligned}
$$

The other coordinates can be obtained by permuting the indices. The local equations of $\bar{Y}_{1}$ in these coordinate patches are

$$
\begin{array}{ll}
\mathcal{U}_{1}: & x_{1} u_{1}+y_{1} v_{1}=\mu, \\
\mathcal{U}_{1}^{\prime}: & x_{1}^{\prime} u_{1}^{\prime}+y_{1}^{\prime}=\mu v_{1}^{\prime}, \\
\mathcal{U}_{1}^{\prime \prime}: & x_{1}^{\prime \prime}+y_{1}^{\prime \prime} v_{1}^{\prime}=\mu u_{1}^{\prime \prime} .
\end{array}
$$

The cycles $\bar{L}_{1}$ and $\bar{L}_{2}$ on $\bar{Y}_{1}$ are given by the following local equations

$$
\begin{array}{lll}
\bar{L}_{1}: & u_{1}=\bar{x}_{1}, & v_{1}=\bar{y}_{1}, \\
\bar{L}_{2}: & u_{2}=\bar{x}_{2}, & v_{2}=\bar{y}_{2} .
\end{array}
$$


Given these local equations, one can deform the symplectic Kähler structure on $\bar{Y}_{1}$ so that the cycles $\bar{L}_{1}$ and $\bar{L}_{2}$ are Lagrangian. The details are explained in Appendix A of ref. [16]. Therefore, the topological open string A-model is well defined, at least in this limit.

One can check that the triple $\left(\bar{Y}, \bar{L}_{1}, \bar{L}_{2}\right)$ is preserved by a subtorus $\left(S^{1}\right)^{2} \subset G$. In fact for localization computations, it suffices to consider a one-parameter subgroup $T \subset G$ defined by

$$
\left(\mathrm{e}^{\mathrm{i} \phi_{1}}, \mathrm{e}^{\mathrm{i} \phi_{2}}, \ldots, \mathrm{e}^{\mathrm{i} \phi_{7}}\right)=\left(\mathrm{e}^{\mathrm{i} \lambda_{1} \phi}, 1, \mathrm{e}^{\mathrm{i} \lambda_{3} \phi}, 1, \mathrm{e}^{-\mathrm{i} \lambda_{1} \phi}, \mathrm{e}^{-\mathrm{i} \lambda_{3} \phi}, 1\right) .
$$

In the next section, we will determine the structure of $T$-invariant open string morphisms with boundary conditions on $\bar{L}_{1} \cup \bar{L}_{2}$.

\subsection{The fixed loci}

At a first look, one would like to sum over open string maps to the singular hypersurface $\bar{Y}$. This is, however, a very difficult task, since the degeneration considered here is not semistable. Therefore, we do not have a good control of intersection theory even on the moduli space of stable closed string maps. Instead, it is more convenient to consider open string maps to the ambient toric variety $\mathcal{Z}$, as explained in Section 3. In order to obtain numerical invariants, we will have to develop an open string version of the convex obstruction bundle.

First note that the fixed point set of the $T$-action on $\mathcal{Z}$ consists of eight isolated fixed points $P_{1}, P_{2}, P_{1}^{\prime}, P_{2}^{\prime}, P_{1}^{\prime \prime}, \ldots, P_{4}^{\prime \prime}$ and two fixed curves $\overline{P_{3} P_{3}^{\prime}}$ and $\overline{P_{4}^{\prime} P_{4}^{\prime \prime}}$. The presence of fixed curves in the target space will cause some complications at a certain point in our analysis.

Now, let $f: \Sigma_{0, h} \longrightarrow \mathcal{Z}$ be a genus zero open string stable morphism which sends the boundary $\partial \Sigma_{0, h}$ to the cycle $\bar{L}=\bar{L}_{1} \cup \bar{L}_{2}$. We also fix the homology class $\beta=f_{*}\left[\Sigma_{0, h}\right] \in H_{2}(\mathcal{Z}, \mathbb{Z}) \simeq H_{2}(\mathcal{Z}, \bar{L} ; \mathbb{Z})$. The torus action (6.14) induces an action on the moduli space of such maps $\bar{M}_{0, h}(\mathcal{Z}, \bar{L} ; \beta)$. Our goal is to determine the structure of fixed loci for this action. By $T$-invariance, the domain of such a map must be either (i) an annulus or (ii) a nodal bordered Riemann surface $\Sigma_{0, h}=\Sigma_{0} \cup \Delta_{1} \cup \cdots \cup \Delta_{h}$, where $\Sigma_{0}$ is a prestable curve of genus 0 and $\Delta_{1}, \ldots, \Delta_{h}$ are $h$ discs attached to $\Sigma_{0}$ by identifying the origins with the marked points $\left(p_{1}, \ldots, p_{h}\right)$. The data $\left(\Sigma_{0}, p_{1}, \ldots, p_{h}\right)$ defines a prestable marked curve of genus 0 . 
Obviously, the first case can be realized only if $h=2$. Then the image of $f$ should be an invariant holomorphic annulus embedded in $\mathcal{Z}$ with boundary on $\bar{L}$. We will show in the following that there are no such annuli in the present example. In the second case, the morphism $f$ should map $\Sigma_{0}$ to a curve in $\mathcal{Z}$ which is preserved by the subtorus action (6.14). The disc components should be mapped to invariant discs embedded in $\mathcal{Z}$ with boundary on $\bar{L}$. One must also impose a stability constraint on $\left(f, \Sigma_{0, h}\right)$ which makes the automorphism group finite.

It is clear that we should start by identifying the invariant annuli and discs in $\mathcal{Z}$ with boundary on $\bar{L}$. Using an argument similar to ref. [15, Section 5], one can show that any invariant embedding $f: \Sigma_{0, h} \longrightarrow \mathcal{Z}$ can be extended to an invariant embedding $\bar{f}: \bar{\Sigma} \longrightarrow \mathcal{Z}$, where $\Sigma$ is a closed Riemann surface without boundary. Moreover, since we are only interested in discs or annuli, $\bar{\Sigma}$ will be a smooth rational curve. Therefore, we have to find all $T$-invariant smooth rational curves on $\mathcal{Z}$ which intersect the cycles $\bar{L}_{1}$ and $\bar{L}_{2}$ along orbits of the torus action. Since $\bar{L}_{1}$ and $\bar{L}_{2}$ are cycles in $\bar{Y}_{1}$, it follows that any such curve must be in fact contained in $\bar{Y}_{1}$. This reduces the analysis to the local case considered briefly in the previous section. Let us give more details here.

As $\bar{Y}_{1}$ is a toric Fano three-fold, this is a simple question which has been addressed in similar situations in refs. $[15,16]$. In the present case, we find the curves

$$
\begin{array}{lll}
C_{13}^{\prime}: & Z_{1}=U=0, & V Z_{2} Z_{3}=\mu W, \\
C_{14}^{\prime \prime}: & Z_{3}=V=0, & U Z_{1} Z_{4}=\mu W, \\
C_{23}^{\prime \prime}: & Z_{2}=V=0, & U Z_{1} Z_{4}=\mu W, \\
C_{24}^{\prime}: & Z_{4}=U=0, & V Z_{2} Z_{3}=\mu W,
\end{array}
$$

which intersect the cycles $\bar{L}_{1}$ and $\bar{L}_{2}$ along invariant circles as follows

$$
\begin{aligned}
& \Gamma_{1}^{\prime} \equiv C_{13}^{\prime} \cap \bar{L}_{1}=\left\{x_{1}=u_{1}=0,\left|y_{1}\right|=\left|v_{1}\right|=\mu^{1 / 2}\right\}, \quad C_{13}^{\prime} \cap \bar{L}_{2}=\emptyset, \\
& \Gamma_{1}^{\prime \prime} \equiv C_{14}^{\prime \prime} \cap \bar{L}_{1}=\left\{\left|x_{1}\right|=\left|u_{1}\right|=\mu^{1 / 2}, y_{1}=v_{1}=0\right\}, \quad C_{14}^{\prime \prime} \cap \bar{L}_{2}=\emptyset \text {, } \\
& C_{23}^{\prime \prime} \cap \bar{L}_{1}=\emptyset, \quad \Gamma_{2}^{\prime \prime} \equiv C_{23}^{\prime \prime} \cap \bar{L}_{2}=\left\{\left|x_{2}\right|=\left|u_{2}\right|=\mu^{1 / 2}, y_{2}=v_{2}=0\right\} \text {, } \\
& C_{24}^{\prime} \cap \bar{L}_{1}=\emptyset, \quad \Gamma_{2}^{\prime} \equiv C_{24}^{\prime} \cap \bar{L}_{2}=\left\{x_{2}=u_{2}=0,\left|y_{2}\right|=\left|v_{2}\right|=\mu^{1 / 2}\right\} .
\end{aligned}
$$

Note that all circles are algebraic knots with orientation induced by the canonical orientation of $\mathcal{Z}$. Moreover, $\Gamma_{1}$ and $\Gamma_{1}^{\prime}$, respectively, $\Gamma_{2}^{\prime}$ and $\Gamma_{2}^{\prime \prime}$ form Hopf links in $\bar{L}_{1}$ and $\bar{L}_{2}$ with linking number +1 . For later use, let us 
introduce the holonomy variables

$$
V_{i}^{\prime}=\mathrm{P} \exp \int_{\Gamma_{i}^{\prime}} A^{(i)}, \quad i=1,2, \quad V_{i}^{\prime \prime}=\mathrm{P} \exp \int_{\Gamma_{i}^{\prime \prime}} A^{(i)}, \quad i=1,2,
$$

where $A^{(1)}$ and $A^{(2)}$ are the Chern-Simons gauge fields on $\bar{L}_{1}$ and $\bar{L}_{2}$. Note that the curves $C_{13}^{\prime}, \ldots, C_{24}^{\prime}$ are invariant under the $T$-action on $\mathcal{Z}$, but not under the generic $G$-action.

Each knot divides one of the curves (equation (6.15)) into two invariant discs embedded in $\mathcal{Z}$ with boundary on $\bar{L}_{1}$ or $\bar{L}_{2}$ as follows

$$
\begin{aligned}
& C_{13}^{\prime}=D_{1}^{\prime} \cup_{\Gamma_{1}^{\prime}} D_{3}^{\prime}, \\
& C_{14}^{\prime \prime}=D_{1}^{\prime \prime} \cup_{\Gamma_{1}^{\prime \prime}} D_{4}^{\prime \prime}, \\
& C_{23}^{\prime \prime}=D_{2}^{\prime \prime} \cup_{\Gamma_{2}^{\prime \prime}} D_{3}^{\prime \prime}, \\
& C_{24}^{\prime}=D_{2}^{\prime} \cup_{\Gamma_{2}^{\prime}} D_{4}^{\prime} .
\end{aligned}
$$

Note that $D_{1}^{\prime}, D_{1}^{\prime \prime}, D_{2}^{\prime}$ and $D_{2}^{\prime \prime}$ intersect the toric skeleton at the fixed points $P_{1}^{\prime}, P_{1}^{\prime \prime}$ and $P_{2}^{\prime}, P_{2}^{\prime \prime}$ lying on the divisor at infinity $\zeta_{\infty}$. The discs $D_{3}^{\prime}, D_{3}^{\prime \prime}$ and, respectively, $D_{4}^{\prime}$ and $D_{4}^{\prime \prime}$ have common origins $Q_{3}$ and $Q_{4}$ given by

$$
\begin{aligned}
& D_{3}^{\prime} \cap D_{4}^{\prime}=\left\{Z_{1}=Z_{4}=U=0, V Z_{2} Z_{3}=\mu W\right\}=Q_{3}, \\
& D_{3}^{\prime \prime} \cap D_{4}^{\prime \prime}=\left\{Z_{2}=Z_{3}=V=0, U Z_{1} Z_{4}=\mu W\right\}=Q_{4} .
\end{aligned}
$$

Note that $Q_{3}$ and $Q_{4}$ lie on the $G$-invariant lines $\overline{P_{3} P_{3}^{\prime}}$ and $\overline{P_{4} P_{4}^{\prime}}$, but they are not fixed points of the $G$-action. The resulting configuration of invariant discs is represented in figure 7 .

For future reference, let us determine the relative homology classes of the discs (equation (6.18)). As noticed earlier, we have $H_{2}(\mathcal{Z}, \bar{L} ; \mathbb{Z}) \simeq H_{2}(\mathcal{Z}, \mathbb{Z})$, which is generated by the curve classes $h_{1}, h_{2}$ and $h_{3}$ specified in equation (6.9). The homology classes of the discs can be determined by deforming to the singular hypersurface $Y_{0}$ as discussed in Section 5 of [15]. In the present case, we obtain

$$
\left[D_{1}^{\prime}\right]=\left[D_{1}^{\prime \prime}\right]=\left[D_{2}^{\prime}\right]=\left[D_{2}^{\prime \prime}\right]=h_{1}, \quad\left[D_{3}^{\prime}\right]=\left[D_{3}^{\prime \prime}\right]=h_{2}, \quad\left[D_{4}^{\prime}\right]=\left[D_{4}^{\prime \prime}\right]=h_{3} .
$$

Adopting the same terminology, we will call the discs $D_{1}^{\prime}, D_{1}^{\prime \prime}, D_{2}^{\prime}$ and $D_{2}^{\prime \prime}$ vertical discs and $D_{3}^{\prime}, D_{3}^{\prime \prime}, D_{4}^{\prime}$ and $D_{4}^{\prime \prime}$ horizontal discs. The homology classes are represented in figure 7 using the color coding introduced below (6.9).

From equation (6.20), it follows that the symplectic areas of the discs are given at the classical level ${ }^{7}$ by the closed string Kähler parameters $t_{1}, t_{2}$ and $t_{3}$. If open string quantum effects are taken into account, the symplectic area

\footnotetext{
${ }^{7}$ What is meant here is classical level from the open string point of view. The parameters $t_{1}, t_{2}$ and $t_{3}$ are flat coordinates on the closed string Kähler moduli space.
} 


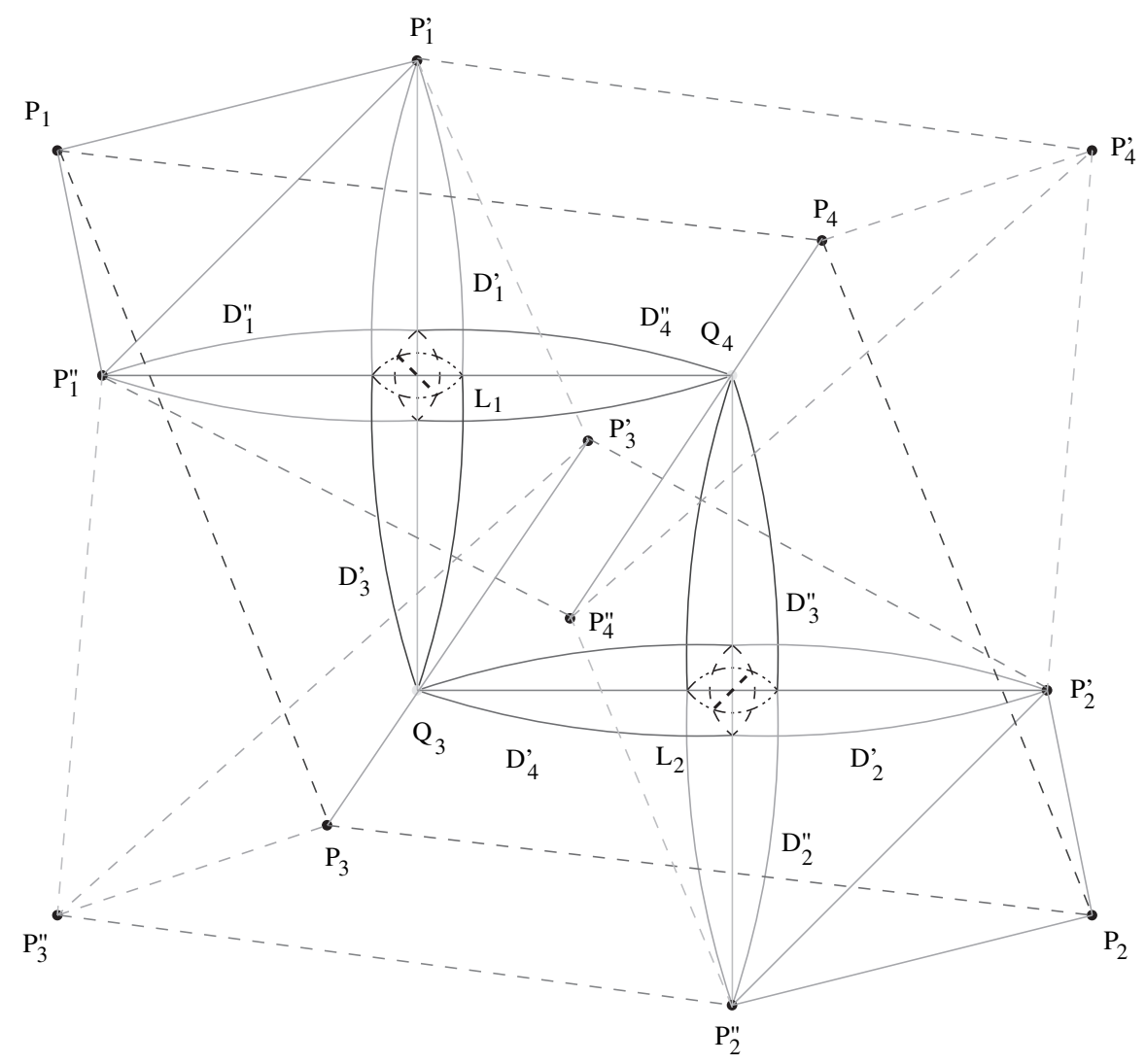

Figure 7: Discs in $\mathcal{Z}$ invariant under the restricted torus action. The points $Q_{3}$ and $Q_{4}$ have been represented by yellow dots in order to distinguish them from the fixed points under the generic torus action. The color coding for discs is the same as for curve classes: green $=h_{1}$, blue $=h_{2}$, red $=h_{3}$.

is shifted by a multiple of $\lambda / 2$ (recall that $\lambda$ is the 't Hooft coupling constant) as discussed in ref. [15]. Therefore, we introduce the corrected Kähler parameters $\tau_{i}^{\prime}, \tau_{i}^{\prime \prime}, i=1, \ldots, 4$, for the eight discs. The relation between $\tau_{i}^{\prime}$, $\tau_{i}^{\prime \prime}$ and the closed string Kähler parameters on the small resolution $\tilde{Y}$ is part of the prescriptions of the duality map, and will be discussed later.

Having determined all invariant discs in $\mathcal{Z}$, we can now understand the structure of a more general invariant map $f: \Sigma_{0, h} \longrightarrow \mathcal{Z}$.

As stated above, the domain $\Sigma_{0, h}$ is the union of a prestable curve $\Sigma_{0}$ of genus zero with $h$ marked points $p_{1}, \ldots, p_{h}$ and $h$ discs $\Delta_{1}, \ldots, \Delta_{h}$ attached to $\Sigma_{0}$ at $p_{1}, \ldots, p_{h}$. Each disc $\Delta_{a}$ is mapped $d_{a}: 1$ to one of the eight discs found above. The closed curve $\Sigma_{0}$ is mapped to a genus zero curve in $\mathcal{Z}$ 
which is preserved by the restricted torus action (6.14). As we are working with the restricted torus action, there may be nontrivial families of invariant curves on $\mathcal{Z}$. Therefore, the connected components of the fixed loci may have a complicated structure, as discussed in more detail below.

We are interested in computing all open string instanton corrections for a fixed relative homology class $\beta \in H_{2}(Y, L ; \mathbb{Z}) \simeq H_{2}(\mathcal{Z}, \bar{L} ; \mathbb{Z})$. Therefore, we have to sum over all fixed loci in $\bar{M}_{0, h}(\mathcal{Z}, \bar{L} ; \beta)$ which are compatible with the given homology class $\beta$. In order to make this sum more explicit, first we have to classify the fixed loci according to the images of the discs $\Delta_{a}$ in $\mathcal{Z}$. There are eight invariant discs $D_{1}^{\prime}, \ldots, D_{4}^{\prime}, D_{1}^{\prime \prime}, \ldots, D_{4}^{\prime \prime}$ embedded in $\mathcal{Z}$, and in principle any such disc can be the image of any component $\Delta_{a}$ of the domain. Let us introduce the set of labels $I=\left\{1^{\prime}, \ldots, 4^{\prime}, 1^{\prime \prime}, \ldots, 4^{\prime \prime}\right\}$ for the invariant discs in $\mathcal{Z}$. The notation is self-explanatory. Specifying the image of each component $\Delta_{a}$ is equivalent to giving a function $\rho:\{1, \ldots, h\} \longrightarrow I$, and we have to sum over all such functions. The instanton series can be written schematically as follows

$$
\sum_{h=1}^{\infty} \sum_{\left(d_{a}, \beta\right)} \sum_{\rho} C_{h, \rho}\left(d_{a}, \beta\right) \mathrm{e}^{-\left\langle J, \beta-\sum_{a=1}^{h} d_{a}\left[D_{\rho(a)}\right]\right\rangle} \mathrm{e}^{-\sum_{a=1}^{h} d_{a} \tau_{\rho(a)}} \prod_{a=1}^{h} \operatorname{Tr} V_{\rho(a)}^{d_{a}} .
$$

Let us explain this formula in detail. The coefficients $C_{h, \rho}\left(d_{a}, \beta\right)$ represent the sum over all fixed loci with given $(h, \rho)$ and $\left(d_{a}, \beta\right)$. We have suppressed the genus subscript $g$ since we will exclusively consider genus zero maps. The exponential factors $\mathrm{e}^{-\left\langle J, \beta-\sum_{a=1}^{h} d_{a}\left[D_{\rho(a)}\right]\right\rangle} \mathrm{e}^{-\sum_{a=1}^{h} d_{a} \tau_{\rho(a)}}$ represent the instanton factors associated to a given map. Naively, these factors should be written as $\mathrm{e}^{-\langle J, \beta\rangle}$, where the pairing in the exponent is well defined since $\left.J\right|_{\bar{L}}=0$ by the Lagrangian condition. We have modified this expression in order to allow for a possible shift in the open string Kähler moduli $\tau_{\rho(a)}$ with respect to the closed string parameters. This shift can be interpreted as an open string quantum effect and plays an important role in the duality map [15]. Therefore, one has to write down separate expressions for the instanton factors of the open and closed string components, obtaining the expression in equation (6.21). The area of the closed component $-\left\langle J, \beta-\sum_{a=1}^{h} d_{a}\left[D_{\rho(a)}\right]\right\rangle$ must be expressed in terms of the closed string parameters $t_{1}, t_{2}$ and $t_{3}$. Finally, $V_{\rho(a)}$ represents the holonomy variable associated to the knot $\Gamma_{\rho(a)}=\partial D_{\rho(a)}$, which is defined as follows. If $\rho(a) \in\left\{1^{\prime}, 1^{\prime \prime}, 2^{\prime}, 2^{\prime \prime}\right\}$, say $\rho(a)=1^{\prime}, V_{\rho(a)}=V_{1}^{\prime}$ is the holonomy of the $U(N)$ connection about the knot $\Gamma_{1}^{\prime}$ defined in equation (6.17). Note that the canonical orientation on $D_{1}^{\prime}$ induces an orientation on $\Gamma_{1}^{\prime}$ using the inner normal convention. The holonomy $V_{1}^{\prime}$ is computed with respect to this particular orientation. This convention is valid for all vertical discs 
$D_{1}^{\prime}, D_{1}^{\prime \prime}, D_{2}^{\prime}$ and $D_{2}^{\prime \prime}$. If $\rho(a) \in\left\{3^{\prime}, 3^{\prime \prime}, 4^{\prime}, 4^{\prime \prime}\right\}, V_{\rho(a)}=V_{i-2}^{-1}, \quad i=3,4$, since the canonical orientation on $D_{3}^{\prime}, \ldots, D_{4}^{\prime \prime}$ induces the opposite orientation on $\Gamma_{1}^{\prime}, \ldots, \Gamma_{2}^{\prime \prime}$ (see figure 7 ).

Our main problem is to evaluate the coefficients $C_{h, \rho}\left(d_{a}, \beta\right)$ using open string localization techniques. This requires a thorough understanding of the deformation complex and the obstruction bundle for open string morphisms.

\subsection{Deformation complex and obstruction bundle for open strings morphisms}

To begin with, let us consider the definition of the instanton coefficients $C_{h, \rho}\left(d_{a}, \beta\right)$ in more detail. We stress that the following considerations are only heuristic arguments based on unproven assumptions. Essentially, we will assume that certain known results in the theory of closed string GromovWitten invariants $[9,10,20,44]$ carry over to the open string case. As in refs. $[25,33]$, these arguments will eventually lead us to a precise computational definition of the enumerative invariants $C_{h, \rho}\left(d_{a}, \beta\right)$. The first assumption is that the moduli space (or, more precisely, the moduli stack) $\bar{M}_{0, h}(\mathcal{Z}, \bar{L} ; \beta)$ exists in the appropriate category, and can be endowed with a perfect obstruction complex. Then one would have a virtual class $\left[\bar{M}_{0, h}(\mathcal{Z}, \bar{L} ; \beta)\right]$ (of positive dimension, since $Z$ is not a Calabi-Yau three-fold). Following the closed string approach, we would also need an obstruction bundle $\mathcal{V}$ on $\bar{M}_{0, h}(\mathcal{Z}, \bar{L} ; \beta)$ of rank equal to the dimension of $\left[\bar{M}_{0, h}(\mathcal{Z}, \bar{L} ; \beta)\right]$. Assuming these elements can be constructed, we could define 'open string Gromov-Witten invariants' by pairing the Euler class $e(\mathcal{V})$ with the virtual fundamental class. However, even in such an ideal situation the coefficients $C_{h, \rho}\left(d_{a}, \beta\right)$ could not be simply defined by this pairing for reasons explained in Section 3. In order to write down the couplings to Chern-Simons theory, one needs in fact an equivariant refinement, as discussed below.

The holonomy $V$ about the boundary $\Gamma$ of an open string surface is defined with respect to an arbitrary (not necessarily flat) $U(N)$ connection. Therefore, it depends continuously on the position of the boundary $\Gamma$ in the cycle $\bar{L}$. In formula $(6.21)$, the $V_{i}$ are holonomy variables about the boundaries $\Gamma_{i}$ of the $T$-invariant discs in $\mathcal{Z}$. Hence the torus action on $(\mathcal{Z}, \bar{L})$ enters in a crucial way. This suggests that the appropriate refinement of open string Gromov-Witten invariants should be considered in an equivariant setting. The torus action on $(\mathcal{Z}, \bar{L})$ induces a torus action on $\bar{M}_{0, h}(\mathcal{Z}, \bar{L} ; \beta)$. Under the above assumptions, we can define equivariant open string Gromov-Witten invariants by working with the equivariant virtual cycle $\left[\bar{M}_{0, h}(\mathcal{Z}, \bar{L} ; \beta)\right]_{T}$ and equivariant obstruction bundle $\mathcal{V}_{T}$. 
Following this line of argument, one would finally have a localization formula for the equivariant invariants of the form

$$
\int_{\left[\bar{M}_{0, h}(\mathcal{Z}, \bar{L} ; \beta)\right]_{T}} e_{T}(\mathcal{V})=\sum_{\Xi} \int_{[\Xi]_{T}} \frac{e_{T}\left(\mathcal{V}_{\Xi}\right)}{e_{T}\left(N_{\Xi}\right)} .
$$

In this formula, we sum over all fixed loci $\Xi$ in the moduli space of open string maps. $e_{T}\left(\mathcal{V}_{\Xi}\right)$ is the equivariant Euler class of the obstruction bundle $\mathcal{V}_{T}$ restricted to $\Xi$ and $e_{T}\left(N_{\Xi}\right)$ is the equivariant Euler class of the virtual normal bundle $N(\Xi)$ to $\Xi$ in $\bar{M}_{0, h}(\mathcal{Z}, \bar{L} ; \beta)$. $[\Xi]_{T}$ is the equivariant fundamental class of the fixed locus $\Xi$. The intersection pairing in the left-hand side of equation (6.22) takes values in the cohomology $\operatorname{ring} \mathcal{R}_{T}=H^{*}(B T) \simeq$ $H^{*}\left(B S^{1}\right)$. The local contributions in the right-hand side take values in the associated fraction field $\mathcal{K}_{T}$.

Now, the fixed loci $\Xi$ can be classified according to the degrees $d_{a}$ and the map $\rho$ as discussed in the paragraph below equation (6.21). The refinement we are looking for is defined by summing only over those fixed loci $\Xi$ with given $\left(\rho, d_{a}\right)$. We will refer to these loci as fixed loci of type $\left(\rho, d_{a}\right)$. The maps therein will also be referred to as open string maps of type $\left(\rho, d_{a}\right)$. We define

$$
\mathcal{C}_{h, \rho}\left(d_{a}, \beta\right)=\sum_{\Xi} \int_{[\Xi]_{T}} \frac{e_{T}\left(\mathcal{V}_{\Xi}\right)}{e_{T}\left(N_{\Xi}\right)}
$$

where the sum is restricted to fixed loci of type $\left(\rho, d_{a}\right)$. Clearly, the resulting invariants $\mathcal{C}_{h, \rho}\left(d_{a}, \beta\right)$ take values in $\mathcal{K}_{T}$. The instanton coefficients $C_{h, \rho}\left(d_{a}, \beta\right)$ are defined as the nonequivariant limit of $\mathcal{C}_{h, \rho}\left(d_{a}, \beta\right)$ [12]. More precisely, let $i_{p t}:\{p t\} \longrightarrow B T$ be the embedding of a point in $B T$. Then we define $C_{h, \rho}\left(d_{a}, \beta\right)$ by

$$
C_{h, \rho}\left(d_{a}, \beta\right)=i_{p t}^{*} \mathcal{C}_{h, \rho}\left(d_{a}, \beta\right) \in H^{*}(p t) .
$$

If we identify $H^{*}(p t)$ with $\mathbb{Q}$, we can regard $C_{h, \rho}\left(d_{a}, \beta\right)$ as a rational function of the toric weights $\lambda_{1}$ and $\lambda_{3}$. In the following, it is very convenient to regard $\lambda_{1}$ and $\lambda_{3}$ as formal variables. This is our heuristic formula for the instanton coefficients. Besides lacking a rigorous formulation, this expression raises another question. If $C_{h, \rho}\left(d_{a}, \beta\right)$ are to be thought of as rational functions of formal variables, what is the physical meaning of the resulting open string expansion? This question has been given an elegant answer in the previous section in the context of local transitions. There we found that the framing in Chern-Simons theory should also be treated as a formal variable, which is related to the toric weights. The final result was shown to be a series with numerical coefficients, as expected. We will show in the next section that similar considerations can be applied to compact models as well, although the final picture will be more subtle in that case. 
At this point, we still have to set the formula (6.23) on firmer grounds. The main idea is to rewrite the open string formula (6.22) in terms of equivariant integrals on moduli spaces of closed string maps with marked points. This approach has been successfully employed in a local context in ref. [25]. The extension to compact three-folds will involve some additional (sometimes delicate) steps. Although our arguments are mostly heuristic, we will eventually obtain well-defined integrals on well-defined moduli spaces. Following the strategy of refs. [25,33], we proceed with the analysis of the deformation-obstruction complex for open string morphisms.

In order to simplify the exposition, let us first consider the case $h=1$, i.e., the domain $\Sigma_{0,1}$ has a single disc component $\Delta$. After a detailed treatment of this case, we will consider the generalization to $h>1$. The disc $\Delta$ can be mapped to any of the discs $D_{i}^{\prime}$ and $D_{i}^{\prime \prime}$, hence we should consider all these cases separately. In fact, it suffices to consider only a vertical disc, say $f(\Delta)=D_{1}^{\prime}$, and a horizontal one, $f(\Delta)=D_{3}^{\prime}$, since all other cases can be treated by analogy.

\subsubsection{Vertical discs}

Let us start with $f(\Delta)=D_{1}^{\prime}$. We have an invariant map $f: \Sigma_{0,1} \longrightarrow \mathcal{Z}$, where $\Sigma_{0,1}=\Sigma_{0} \cup_{p} \Delta$ and $\Sigma_{0}$ is a prestable curve of genus zero with a marked point $p$. We denote by $f_{0}, f_{\Delta}$ the restrictions of $f$ to $\Sigma_{0}$ and, respectively, $\Delta$, and by $f_{\partial}$ the restriction of $f$ to the boundary $\partial \Sigma=\partial \Delta$. Moreover, from now on we will suppress the subscript $(0,1)$ on $\Sigma$. In local coordinates, $f_{\Delta}$ is given by

$$
x_{1}^{\prime}=u_{1}^{\prime}=0, \quad y_{1}^{\prime}=v_{1}^{\prime}=t^{\prime d_{1}^{\prime}}
$$

where $t^{\prime}$ is a local coordinate on $\Delta$. Note that there is an induced torus action on $\Delta$ with weight $\lambda_{t^{\prime}}=\lambda_{v_{1}^{\prime}} / d_{1}^{\prime}$.

The map $f$ represents a point in some component $\Xi$ of the fixed locus. Following our main strategy, we would like to identify $\Xi$ with a component $\Xi_{0}$ of the fixed locus in a certain moduli space of stable closed string maps. Note that the triple $\left(\Delta, f_{\Delta}, p\right)$ defines an isolated fixed point in the moduli space of $d_{1}^{\prime}: 1$ multicovers of the disc $D_{1}^{\prime}$ with a marked point.

If $\Sigma_{0}$ is empty, this is in fact an isolated fixed point in the moduli space of open string maps $\bar{M}_{0,1}\left(\mathcal{Z}, \bar{L} ; d_{1}^{\prime} h_{1}\right)$, since $\left[D_{1}^{\prime}\right]=h_{1}$, as explained in the paragraph containing equation (6.20). The associated coefficient $\mathcal{C}_{1,1^{\prime}}\left(d_{1}^{\prime}, d_{1}^{\prime} h_{1}\right)$ is evaluated in Appendix B. We will shortly review some aspects of that computation. 
If $\Sigma_{0}$ is not empty, the triple $\left(\Sigma_{0}, f_{0}, p\right)$ is a stable closed string map to $\mathcal{Z}$ with a marked point subject to the constraint $f(p)=P_{1}^{\prime} \in \mathcal{Z}$. Moreover, the homology class of $f_{0}$ is determined by $\beta^{\prime}=\beta-d_{1}^{\prime} h_{1} \in H_{2}(\mathcal{Z}, \mathbb{Z})$. Therefore, $\left(\Sigma_{0}, f_{0}, p\right)$ represents a fixed point in a closed subspace $\bar{M}_{P_{1}^{\prime}}$ of the moduli space of stable closed string maps $\bar{M}_{0,1}\left(\mathcal{Z}, \beta^{\prime}\right)$ defined by the following commutative diagram

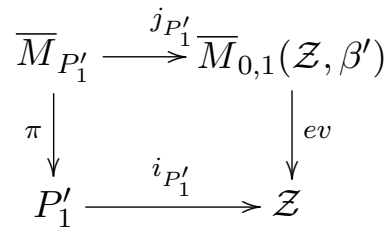

Note that the $T$-action on $\bar{M}_{0,1}\left(\mathcal{Z}, \beta^{\prime}\right)$ induces a $T$-action on $\bar{M}_{P_{1}^{\prime}}$ since $P_{1}^{\prime}$ is a fixed point on $\mathcal{Z}$. In principle, $\left(\Sigma_{0}, f_{0}, p\right)$ is not an isolated fixed point, but it belongs to a component $\Xi_{0}$ of the fixed locus of the induced $T$-action on $\bar{M}_{P_{1}^{\prime}}$. By construction, there is an obvious 1:1 map between $\psi: \Xi \longrightarrow \Xi_{0}$ defined by $(\Sigma, f) \longrightarrow\left(\Sigma_{0}, f_{0}, p\right)$. This map is well defined and $1: 1$ since $\left(\Delta, f_{\Delta}, p\right)$ is an isolated fixed point. Now, the fixed locus $\Xi_{0}$ is in fact a closed subspace of the moduli space $\bar{M}_{P_{1}^{\prime}}$ (more precisely, it is a closed algebraic substack) and is equipped with an induced virtual fundamental class $\left[\Xi_{0}\right]=\iota_{\Xi_{0}}\left[\bar{M}_{P_{1}^{\prime}}\right]$. Here $\iota_{\Xi_{0}}: \Xi_{0} \longrightarrow \bar{M}_{P_{1}^{\prime}}$ denotes the embedding map and $\iota_{\Xi_{0}}: A_{*}\left(\bar{M}_{P_{1}^{\prime}}\right) \longrightarrow A_{*}\left(\Xi_{0}\right)$ denotes the associated Gysin map. The virtual cycle $\left[\bar{M}_{P_{1}^{\prime}}\right]$ is induced by the base change diagram (6.26), $\left[\bar{M}_{P_{1}^{\prime}}\right]=$ $i_{P_{1}^{\prime}}^{!}\left[\bar{M}_{0,1}(\mathcal{Z}, \beta)\right]$. All these considerations carry over to the equivariant setting.

As part of our assumptions, let us suppose there is a similar structure on $\Xi$ so that the map $\psi$ described in the above paragraph becomes an isomorphism. Then $\Xi$ will also be endowed with a virtual cycle $\psi^{!}\left[\Xi_{0}\right]$, where $\psi^{!}: A_{*}\left(\Xi_{0}\right) \longrightarrow A_{*}(\Xi)$ is the Gysin map. This is the first ingredient in a rigorous formulation of the integral in equation (6.22). We still need to make sense of the integrand.

To this end, we have to determine the virtual normal bundle $N_{\Xi}$ using the tangent-obstruction complex of $(\Sigma, f)$, and to construct the obstruction bundle $\mathcal{V}$. First we have to introduce some notation. The restriction of the holomorphic tangent bundle $T_{\mathcal{Z}}$ to $\bar{L}_{1}$ admits a real subbundle $T_{\mathbb{R}}$ defined as the fixed locus of the local antiholomorphic involution $\left(x_{1}, y_{1}, u_{1}, v_{1}\right) \longrightarrow$ $\left(\bar{u}_{1}, \bar{v}_{1}, \bar{x}_{1}, \bar{y}_{1}\right)$. We denote by $\mathcal{T}$ the sheaf of sections of Riemann-Hilbert bundle defined by the pair $\left(f^{*} T_{\mathcal{Z}}, f_{\partial}^{*} T_{\mathbb{R}}\right)$ on $\Sigma$. Note that $\left.\mathcal{T}\right|_{\Sigma_{0}}=f_{0}^{*} T_{\mathcal{Z}}$. We will also denote by $\mathcal{T}_{\Delta}$ the restriction of $\mathcal{T}$ to $\Delta$. The deformation complex 
for the map $f$ is of the form

$$
0 \longrightarrow \operatorname{Aut}(\Sigma) \longrightarrow H^{0}(\Sigma, \mathcal{T}) \longrightarrow \mathbb{T}^{1} \longrightarrow \operatorname{Def}(\Sigma) \longrightarrow H^{1}(\Sigma, \mathcal{T}) \longrightarrow \mathbb{T}^{2} \longrightarrow 0
$$

where $\mathbb{T}^{1}$ and $\mathbb{T}^{2}$ are the deformation and, respectively, obstruction spaces of the map $(\Sigma, f)$. Using a normalization exact sequence with respect to the decomposition $\Sigma=\Sigma_{0} \cup_{p} \Delta$, one can split the terms in equation (6.27) into open string and closed string parts, plus corrections due to the node $p$. We will show below that the closed string part reduces to the standard deformation complex for $\Xi_{0}$. The open string part and the node corrections become part of the data of an equivariant integral on $\Xi_{0}$. In order to completely specify the integrand, we also have to construct the obstruction bundle $\mathcal{V}$. As outlined in Section 3, the fiber of the obstruction bundle over a point $(\Sigma, f)$ is given by the space of global holomorphic sections of a Riemann-Hilbert bundle $\mathcal{L}=\left(f^{*} \Lambda^{4}\left(T_{\mathcal{Z}}\right), \mathcal{R}\right)$ on $\Sigma$. The real subbundle $\left.\mathcal{R} \subset f_{\partial}^{*}\left(\Lambda^{4}\left(T_{\mathcal{Z}}\right)\right)\right|_{\bar{L}_{1}}$ is defined as the fixed set of the local antiholomorphic involution $\left(x_{1}, y_{1}, u_{1}, v_{1}\right) \longrightarrow\left(\bar{u}_{1}, \bar{v}_{1}, \bar{x}_{1}, \bar{y}_{1}\right)$. $\mathcal{V}_{\Xi}$ can also be decomposed in closed and, respectively, open string parts using a normalization exact sequence. The terms in equation (6.27) and in the various normalization sequences encountered in the process are not in general vector bundles over the fixed loci because the fiber dimension may jump. Nevertheless, we will formally manipulate such objects as if they were locally free following, e.g., the approach of ref. [36]. This approach yields correct results since it is only the equivariant K-theory class of these objects which enters the computations. Equivariant K-theory classes will be denoted by []. We have to distinguish two cases.

(i) If $\Sigma_{0}$ is empty, there is no closed string part and $\Xi$ is an isolated fixed point. In this case, we will denote $\Xi$ by $\Xi_{\Delta}$ to avoid any confusion with the general case. The deformation complex becomes

$$
0 \longrightarrow \operatorname{Aut}(\Delta) \longrightarrow H^{0}(\Delta, \mathcal{T}) \longrightarrow \mathbb{T}^{1} \longrightarrow 0 \longrightarrow H^{1}(\Delta, \mathcal{T}) \longrightarrow \mathbb{T}^{2} \longrightarrow 0
$$

since the domain $\Delta$ has no deformations. The automorphism group is generated by holomorphic vector fields of the form $a \partial_{t^{\prime}}+b t^{\prime} \partial_{t^{\prime}}$, where $a \in \mathbb{C}$ and $b \in \mathbb{R}$. Therefore, in terms of $T=S^{1}$ representations we have

$$
\operatorname{Aut}(\Delta) \simeq(0)_{\mathbb{R}} \oplus\left(-\lambda_{t^{\prime}}\right)
$$

The cohomology groups $H^{0}(\Delta, \mathcal{T})$ and $H^{1}(\Delta, \mathcal{T})$ are computed in Appendix B. We have $H^{1}(\Delta, \mathcal{T})=0$, hence $\mathbb{T}^{2}=0$ as well, and the complex (6.28) reduces to

$$
0 \longrightarrow \operatorname{Aut}(\Delta) \longrightarrow H^{0}(\Delta, \mathcal{T}) \longrightarrow \mathbb{T}^{1} \longrightarrow 0
$$


The remaining term $H^{0}(\Delta, \mathcal{T})$ decomposes into representations of $T$ as follows

$$
H^{0}(\Delta, \mathcal{T}) \simeq \bigoplus_{n=0}^{d_{1}^{\prime}}\left(-\lambda_{1}+\frac{n}{d_{1}^{\prime}} \lambda_{3}\right) \oplus \bigoplus_{n=0}^{2 d_{1}^{\prime}}\left(\frac{n-d_{1}^{\prime}}{d_{1}^{\prime}} \lambda_{3}\right) .
$$

Note that for $n=d_{1}^{\prime}$ in the second sum we obtain a term of weight zero $(0)_{\mathbb{C}}$ containing the image of the fixed part $(0)_{\mathbb{R}}$ of $\operatorname{Aut}(\Delta)$. It follows from the exact sequence (6.30) that we obtain a term of weight zero $(0)_{\mathbb{R}}$ in the equivariant decomposition of $\mathbb{T}^{1}$. The obstruction space $\mathcal{V}_{\Delta}=H^{0}(\Delta, \mathcal{L})$ is also computed in Appendix B, with the result

$$
H^{0}(\Delta, \mathcal{L})=(0)_{\mathbb{R}} \oplus \bigoplus_{n=1}^{3 d_{1}^{\prime}}\left(\frac{n}{d_{1}^{\prime}} \lambda_{3}\right) .
$$

Note that this is a real vector space containing a fixed direct summand $(0)_{\mathbb{R}}$. The local contribution of such a fixed point should be of the form

$$
\mathcal{C}_{1,1^{\prime}}\left(d_{1}^{\prime}, d_{1}^{\prime} h_{1}\right)=\int_{p t_{T}} e_{T}\left(\left[\mathcal{V}_{\Delta}\right]-\left[N_{\Delta}\right]\right),
$$

where $N_{\Delta}$ is the virtual normal bundle to $\Xi_{\Delta}$. A straightforward extrapolation of standard localization results would predict that $N_{\Delta}$ is given by the moving part of $\mathbb{T}^{1}$. However, we have to be more careful here. Since $\Xi_{\Delta}$ is an isolated fixed point, we will define $N_{\Delta}$ to be the tangent space $\mathbb{T}^{1}$, including the fixed real deformation $(0)_{\mathbb{R}}$ noticed above. The obstruction space contains an identical fixed real summand, and the two terms cancel off in the formula (6.33), leaving a well-defined nonzero answer. This cancellation reflects the fact that the fixed infinitesimal deformation is obstructed. The situation encountered here is a bit unusual compared to standard localization computations for closed string maps, but we do not see other sensible solution for the moment. This unusual behavior is very likely related to the fact that the cycles $\bar{L}$ are not Lagrangian middle dimensional cycles on $\mathcal{Z}$. The solution proposed here should be regarded as an experimental result, which will be backed up by numerical computations in the next section. We leave a more conceptual treatment for future work. ${ }^{8}$ Collecting the facts, this leaves us with the following

\footnotetext{
${ }^{8}$ Another point of view one could take is to consider open string maps to the singular three-fold $\bar{Y}$ instead of $\mathcal{Z}$. The problem is that the vertical discs intersect the singular divisor $D_{\infty}$ on $\bar{Y}$ and it is not clear how to write down the deformation complex of such a map. (Recall that $\bar{Y}$ contains nonreduced components.) This approach has been effectively used for horizontal discs in the local case since they do not intersect the singular locus. According to our calculations in Appendix B, for horizontal discs the two methods give the same answer. This is an encouraging sign, although much more work remains to be done.
} 
formula for $d_{1}^{\prime}$ multicovers of $D_{1}^{\prime}$

$$
\mathcal{C}_{1,1^{\prime}}\left(d_{1}^{\prime}, d_{1}^{\prime} h_{1}\right)=\frac{(-1)^{d_{1}^{\prime}}}{d_{1}^{\prime d_{1}^{\prime}+2}} \frac{\left(3 d_{1}^{\prime}\right) !}{\left(d_{1}^{\prime} !\right)^{2}} \frac{\lambda_{3}^{d_{1}^{\prime}+1}}{\prod_{n=0}^{d_{1}^{\prime}}\left(-\lambda_{1}+\left(n / d_{1}^{\prime}\right) \lambda_{3}\right)} .
$$

There is a subtle sign ambiguity in this expression reflecting the choice of a complex structure on moduli space of open string maps, as explained in Appendix B. In the absence of a more rigorous construction, the only criterion for fixing the sign at present is agreement with the closed string dual. This will be shown in the next section.

(ii) If $\Sigma_{0}$ is not empty, we have to split $\left[\mathbb{T}^{1}\right],\left[\mathbb{T}^{2}\right]$ and $\left[\mathcal{V}_{\Xi}\right]$ in open and closed string parts using the exact sequences

$$
\begin{gathered}
0 \longrightarrow \mathcal{T} \longrightarrow f_{0}^{*} T_{\mathcal{Z}} \oplus \mathcal{T}_{\Delta} \longrightarrow\left(f^{*} T_{\mathcal{Z}}\right)_{p} \longrightarrow 0, \\
0 \longrightarrow \mathcal{L} \longrightarrow f_{0}^{*} \Lambda^{4}\left(T_{\mathcal{Z}}\right) \oplus \mathcal{L}_{\Delta} \longrightarrow\left(f^{*} \Lambda^{4}\left(T_{\mathcal{Z}}\right)\right)_{p} \longrightarrow 0 .
\end{gathered}
$$

The associated long exact sequences read

$$
\begin{aligned}
0 \longrightarrow H^{0}(\Sigma, \mathcal{T}) \longrightarrow H^{0}\left(\Sigma_{0}, f_{0}^{*} T_{\mathcal{Z}}\right) \oplus H^{0}\left(\Delta, \mathcal{T}_{\Delta}\right) \longrightarrow\left(T_{\mathcal{Z}}\right)_{P_{1}^{\prime}} \\
\longrightarrow H^{1}(\Sigma, \mathcal{T}) \longrightarrow H^{1}\left(\Sigma, f_{0}^{*} T_{\mathcal{Z}}\right) \oplus H^{1}\left(\Delta, \mathcal{T}_{\Delta}\right) \longrightarrow 0 \\
0 \longrightarrow H^{0}(\Sigma, \mathcal{L}) \longrightarrow H^{0}\left(\Sigma_{0}, f_{0}^{*} \Lambda^{4}\left(T_{\mathcal{Z}}\right) \oplus H^{0}\left(\Delta, \mathcal{L}_{\Delta}\right) \longrightarrow \Lambda^{4}\left(T_{\mathcal{Z}}\right)_{P_{1}^{\prime}}\right. \\
\longrightarrow H^{1}(\Sigma, \mathcal{L}) \longrightarrow H^{1}\left(\Sigma_{0}, f_{0}^{*} \Lambda^{4}\left(T_{\mathcal{Z}}\right)\right) \oplus H^{1}\left(\Delta, \mathcal{L}_{\Delta}\right) \longrightarrow 0
\end{aligned}
$$

We claim that $H^{1}\left(\Sigma_{0}, f_{0}^{*} \Lambda^{4}\left(T_{\mathcal{Z}}\right)\right)=0$. This follows from a similar normalization sequence applied to the irreducible components of $\Sigma_{0}$. The pull-back $f_{0}^{*} \Lambda^{4}\left(T_{\mathcal{Z}}\right)$ has nonnegative degree on each irreducible component of $\Sigma_{0}$ since $\Lambda^{4}\left(T_{\mathcal{Z}}\right)=\mathcal{O}\left(-K_{\mathcal{Z}}\right)$ is negative on $\mathcal{Z}$. Since all components are rational, the claim follows easily from the associated long exact sequence. With more effort, we can also show that $H^{1}\left(\Delta, \mathcal{L}_{\Delta}\right)=0$ (according to Appendix B), hence the last term of the exact sequence (6.38) is trivial. The morphism $H^{0}\left(\Sigma_{0}, f_{0}^{*} \Lambda^{4}\left(T_{\mathcal{Z}}\right)\right) \oplus H^{0}\left(\Delta, \mathcal{L}_{\Delta}\right)$ $\longrightarrow \Lambda^{4}\left(T_{\mathcal{Z}}\right)_{P_{1}^{\prime}}$ is surjective since $\Sigma_{0}$ has genus zero and $\Lambda^{4}\left(T_{\mathcal{Z}}\right)$ is negative on $\mathcal{Z}$. Therefore, we conclude that $H^{1}(\Sigma, \mathcal{L})=0$, i.e., the obstruction bundle is convex, as promised before. The exact sequence (6.38) reduces to the first three terms.

Moreover, note that $\operatorname{Aut}(\Sigma)$ decomposes as $\operatorname{Aut}\left(\Sigma_{0}, p\right) \oplus \operatorname{Aut}(\Delta, p)$ in automorphisms of $\Sigma_{0}$, respectively, $\Delta$ which preserve the point $p . \operatorname{Def}(\Sigma)$ decomposes similarly as $\operatorname{Def}\left(\Sigma_{0}, p\right) \oplus T_{p} \Sigma_{0} \otimes T_{p} \Delta$ in deformations of $\Sigma_{0}$ leaving $p$ fixed and deformations of the node. There are no deformations of $\Delta$. Using the exact sequences (6.27), (6.37) and (6.38), we find the following 
relations in the equivariant K-theory of $\Xi$

$$
\begin{aligned}
{\left[\mathbb{T}^{1}\right]-\left[\mathbb{T}^{2}\right]=} & {\left[H^{0}(\Sigma, \mathcal{T})\right]-\left[H^{1}(\Sigma, \mathcal{T})\right]+[\operatorname{Def}(\Sigma)]-[\operatorname{Aut}(\Sigma)] } \\
= & {\left[H^{0}\left(\Delta, \mathcal{T}_{\Delta}\right)\right]-[\operatorname{Aut}(\Delta)]+\left[\partial_{t^{\prime}}\right]+\left[H^{0}\left(\Sigma_{0}, f_{0}^{*} T_{\mathcal{Z}}\right)\right] } \\
& -\left[H^{1}\left(\Sigma_{0}, f_{0}^{*} T_{\mathcal{Z}}\right)\right]+\left[\operatorname{Def}\left(\Sigma_{0}, p\right)\right]-\left[\operatorname{Aut}\left(\Sigma_{0}, p\right)\right] \\
& -\left[\left(T_{\mathcal{Z}}\right)_{P_{1}^{\prime}}\right]+\left[T_{p} \Sigma_{0} \otimes T_{p} \Delta\right]
\end{aligned}
$$

and

$$
\left[\mathcal{V}_{\Xi}\right]=\left[H^{0}(\Delta, \mathcal{L})\right]+\left[H^{0}\left(\Sigma_{0}, f_{0}^{*} \Lambda^{4}\left(T_{\mathcal{Z}}\right)\right)\right]-\left[\Lambda^{4}\left(T_{\mathcal{Z}}\right)_{P_{1}^{\prime}}\right]
$$

The combination $\left[H^{0}\left(\Delta, \mathcal{T}_{\Delta}\right)\right]-[\operatorname{Aut}(\Delta)]$ is precisely the K-theory class of the normal bundle $N_{\Delta}$ to the fixed point $\left(\Delta, f_{\Delta}\right)$ defined at point (i) above. In order to identify the remaining terms in equation (6.39), let us write down the tangent-obstruction complex for the stable map $\left(\Sigma_{0}, f_{0}, p\right)$ regarded as a point in $\bar{M}_{0,1}\left(\mathcal{Z}, \beta^{\prime}\right)$

$$
\begin{aligned}
0 \longrightarrow \operatorname{Aut}\left(\Sigma_{0}, p\right) \longrightarrow H^{0}\left(\Sigma_{0}, f_{0}^{*} T_{\mathcal{Z}}\right) \longrightarrow \mathbb{T}_{0}^{1} \longrightarrow \operatorname{Def}\left(\Sigma_{0}, p\right) \\
\quad \longrightarrow H^{1}\left(\Sigma_{0}, f_{0}^{*} T_{\mathcal{Z}}\right) \longrightarrow \mathbb{T}_{0}^{2} \longrightarrow 0 .
\end{aligned}
$$

Using the K-theory relations derived from equation (6.41), we can rewrite equation (6.39) as follows

$$
\left[\mathbb{T}^{1}\right]-\left[\mathbb{T}^{2}\right]=\left[N_{\Delta}\right]+\left[\partial_{t^{\prime}}\right]+\left[\mathbb{T}_{0}^{1}\right]-\left[\mathbb{T}_{0}^{2}\right]-\left[\left(T_{\mathcal{Z}}\right)_{P_{1}^{\prime}}\right]+\left[T_{p} \Sigma_{0} \otimes T_{p} \Delta\right] .
$$

The combination $\left[\mathbb{T}_{0}^{1}\right]-\left[\mathbb{T}_{0}^{2}\right]-\left[\left(T_{\mathcal{Z}}\right)_{P_{1}^{\prime}}\right]$ represents the image in K-theory of the deformation complex of $\left(\Sigma_{0}, f_{0}, p\right)$ regarded as a point in $\bar{M}_{P_{1}^{\prime}}$. This follows from the construction of $\bar{M}_{P_{1}^{\prime}}$ as the subspace of $\bar{M}_{0,1}\left(\mathcal{Z}, \beta^{\prime}\right)$ defined by $f_{0}(p)=P_{1}^{\prime}$. Therefore, the K-theory class of the virtual normal bundle $N_{\Xi_{0}}$ in $\bar{M}_{P_{1}^{\prime}}$ is given by the moving parts

$$
\left[N_{\Xi_{0}}\right]=\left[\left(\mathbb{T}_{0}^{1}\right)^{m}\right]-\left[\left(\mathbb{T}_{0}^{2}\right)^{m}\right]-\left[\left(\left(T_{\mathcal{Z}}\right)_{P_{1}^{\prime}}\right)^{m}\right] .
$$

We will define the class of the open string virtual normal bundle $N_{\Xi}$ to be

$$
\begin{aligned}
{\left[N_{\Xi}\right]=} & {\left[N_{\Delta}\right]+\left[\left(\mathbb{T}_{0}^{1}\right)^{m}\right]-\left[\left(\mathbb{T}_{0}^{2}\right)^{m}\right]-\left[\left(\left(T_{\mathcal{Z}}\right)_{P_{1}^{\prime}}\right)^{m}\right]+\left[\left(\partial_{t^{\prime}}\right)^{m}\right] } \\
& +\left[\left(T_{p} \Sigma_{0} \otimes T_{p} \Delta\right)^{m}\right] .
\end{aligned}
$$

Up to the first term, which has been explained above, this is just the moving part of equation (6.42), as expected. The fixed part of equation (6.42), $\left[\left(\mathbb{T}_{0}^{1}\right)^{f}\right]-\left[\left(\mathbb{T}_{0}^{2}\right)^{f}\right]-\left[\left(\left(T_{\mathcal{Z}}\right)_{P_{1}^{\prime}}\right)^{f}\right]$, enters the construction of the induced virtual cycle $\left[\Xi_{0}\right]_{T} \in A_{*}^{T}\left(\Xi_{0}\right),\left[\Xi_{0}\right]_{T}=\iota_{\Xi_{0}}\left[\bar{M}_{P_{1}^{\prime}}\right]_{T}[20]$. This is consistent with our earlier proposal $[\Xi]=\psi !\left[\Xi_{0}\right]$ for the open string virtual cycle (see the paragraph below equation (6.26)).

The K-theory class of the obstruction bundle splits similarly in an open string part $\left[\mathcal{V}_{\Delta}\right]$ which has been considered before, and a closed string part 
$\left[H^{0}\left(\Sigma_{0}, f_{0}^{*} \Lambda^{4}\left(T_{\mathcal{Z}}\right)\right)\right]$. The later is the K-theory class of the standard obstruction bundle over $\bar{M}_{0,1}\left(\mathcal{Z}, \beta^{\prime}\right)$ restricted to $\Xi_{0}$. Recall that the fiber of $\mathcal{V}^{0}$ over a point $\left(\Sigma_{0}, f_{0}, p\right)$ is given precisely by $H^{0}\left(\Sigma_{0}, f_{0}^{*} \Lambda^{4}\left(T_{\mathcal{Z}}\right)\right)$.

We can now tie all loose ends together and write down a well-defined local formula for the open string invariants $\mathcal{C}_{1,1^{\prime}}\left(d_{1}^{\prime}, \beta\right)$

$$
\frac{\mathcal{C}_{1,1^{\prime}}\left(d_{1}^{\prime}, d_{1}^{\prime} h_{1}\right)}{\left(-\lambda_{t^{\prime}} H\right)} \int_{\left[\Xi_{0}\right]_{T}} \frac{1}{e_{T}\left(N_{\Xi_{0}}\right)} \frac{e_{T}\left(\left[\mathcal{V}_{\Xi_{0}}^{0}-\Lambda^{4}\left(T_{\mathcal{Z}}\right)_{P_{1}^{\prime}}\right]\right)}{-\lambda_{t^{\prime}} H-\psi_{p}}
$$

where $H$ is the generator of $H^{*}(B T)$. The factor $\left(-\lambda_{t^{\prime}} H\right)$ in the denominator represents the equivariant Euler class of $\left[\partial_{t^{\prime}}\right], \psi_{p}$ is the Mumford class associated to the point $p$ and $\Lambda^{4}\left(T_{\mathcal{Z}}\right)_{P_{1}^{\prime}}$ should be regarded as an equivariant bundle over $\Xi_{0}$. Summing over all fixed loci $\Xi_{0}$, we obtain the following expression

$$
\mathcal{C}_{1,1^{\prime}}\left(d_{1}^{\prime}, \beta\right)=\frac{\mathcal{C}_{1,1^{\prime}}\left(d_{1}^{\prime}, d_{1}^{\prime} h_{1}\right)}{\left(-\lambda_{t^{\prime}} H\right)} \int_{\left[\bar{M}_{P_{1}^{\prime}}\right]_{T}} \frac{e_{T}\left(\left[j_{P_{1}^{\prime}}^{*}\left(\mathcal{V}^{0}\right)-\Lambda^{4}\left(T_{\mathcal{Z}}\right)_{P_{1}^{\prime}}\right]\right)}{-\lambda_{t^{\prime}} H-\psi_{p}}
$$

where $\Lambda^{4}\left(T_{\mathcal{Z}}\right)_{P_{1}^{\prime}}$ should be regarded as an equivariant bundle over $\bar{M}_{P_{1}^{\prime}}$.

Although this formula is now well defined, it is also of little use for explicit computations. The major problem is that the fixed loci $\Xi_{0}$ can have a very complicated structure, since the $T$ action on $\mathcal{Z}$ is not generic. Ideally, we would like to be able to compute the invariants (6.46) in terms of standard Kontsevich graphs for closed string maps to $\mathcal{Z}$. In order to do so, we have to take one more step and rewrite the formula (6.46) in terms of a generic torus action. Recall that $T$ is a subgroup of the torus $G=\left(S^{1}\right)^{7}$ which acts on $\mathcal{Z}$ and $P_{1}^{\prime}$ is a $G$-fixed point on $\mathcal{Z}$. Then we have the following commutative diagram

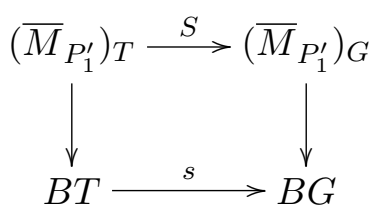

Let $\left(\eta_{1}, \ldots, \eta_{7}\right)$ denote the generators of $H^{*}(B G)$ defined by the characters of $G$ as explained in Chapter 9 of ref. [12]. Then we have

$$
\mathcal{K}_{G} \simeq \mathbb{Q}\left(\eta_{1}, \ldots, \eta_{7}\right), \quad \mathcal{K}_{T} \simeq \mathbb{Q}\left(\lambda_{1} H, \lambda_{3} H\right)
$$


The map $s$ in the above diagram induces a pull-back map $s^{*}: \mathcal{K}_{G} \longrightarrow \mathcal{K}_{T}$ by localization, which factorizes as

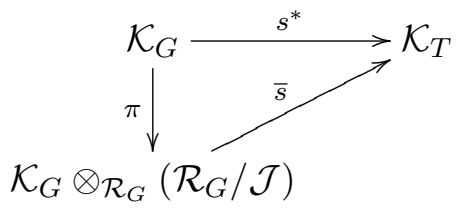

where $\mathcal{J} \subset \mathcal{R}_{G}$ is the ideal generated by $\left(\eta_{2}, \eta_{4}, \eta_{1}+\eta_{5}, \eta_{3}+\eta_{6}, \eta_{7}\right)$, and $\bar{s}$ is defined by $\bar{s}\left(\eta_{1}\right)=\lambda_{1} H$ and $\bar{s}\left(\eta_{3}\right)=\lambda_{3} H$. Using these relations, we can write the class $-\lambda_{t^{\prime}} H-\psi_{p}=-\left(\lambda_{3} / d_{1}^{\prime}\right) H-\psi_{p}$ in equation (6.46) as $s^{*}\left(-\eta_{3} / d_{1}^{\prime}-\psi_{p}\right)$. Moreover, the equivariant virtual class $\left[\bar{M}_{P_{1}^{\prime}}\right]_{T}$ and the obstruction bundle $\mathcal{V}^{0}$ are pulled back via $S$ from $\left(\bar{M}_{P_{1}^{\prime}}\right)_{G}$ (for the virtual cycles, we have to use the Gysin map). Therefore, using the projection formula, we can rewrite (6.46) in the form

$$
\mathcal{C}_{1,1^{\prime}}\left(d_{1}^{\prime}, \beta\right)=\frac{\mathcal{C}_{1,1^{\prime}}\left(d_{1}^{\prime}, d_{1}^{\prime} h_{1}\right)}{\left(-\lambda_{t^{\prime}} H\right)} s^{*}\left[\int_{\left[\bar{M}_{P_{1}^{\prime}}\right]_{G}} \frac{e_{G}\left(\left[j_{P_{1}^{\prime}}^{*}\left(\mathcal{V}^{0}\right)-\Lambda^{4}\left(T_{\mathcal{Z}}\right)_{P_{1}^{\prime}}\right]\right)}{-\eta_{3} / d_{1}^{\prime}-\psi_{p}}\right] .
$$

We will explain how to perform explicit computations using this formula in the next section. Here let us note that we can rewrite equation (6.50) in a different form which is closer to the integrals written in ref. [25]

$$
\begin{aligned}
\mathcal{C}_{1,1^{\prime}}\left(d_{1}^{\prime}, \beta\right)= & \frac{\mathcal{C}_{1,1^{\prime}}\left(d_{1}^{\prime}, d_{1}^{\prime} h_{1}\right)}{\left(-\lambda_{t^{\prime}} H\right)} s^{*}\left[\frac{1}{e_{G}\left(\Lambda^{4}\left(T_{\mathcal{Z}}\right)_{P_{1}^{\prime}}\right)}\right. \\
& \left.\times \int_{\left[\bar{M}_{0,1}\left(\mathcal{Z}, \beta^{\prime}\right)\right]_{G}} \frac{e_{G}\left(\mathcal{V}^{0}\right) e v_{G}^{*}\left(\phi_{P_{1}^{\prime}}\right)}{-\eta_{3} / d_{1}^{\prime}-\psi_{p}}\right],
\end{aligned}
$$

where $e v_{G}: \bar{M}_{0,1}\left(\mathcal{Z}, \beta^{\prime}\right)_{G} \longrightarrow \mathcal{Z}_{G}$ is the equivariant evaluation map and $\phi_{P_{1}^{\prime}} \in$ $H_{G}^{*}(\mathcal{Z})$ the equivariant Thom class of the fixed point $P_{1}^{\prime}$. The equivalence of the two expressions follows from the commutative diagram (6.26) by a series of formal manipulations based on the functorial properties of Chow groups given, e.g., in ref. [46, Chapter V, Sections 5-8]. Let $\alpha=\frac{e_{G}\left(\mathcal{V}^{0}\right)}{-\left(\eta_{3} / d_{1}^{\prime}-\psi_{p}\right)} \in$ $A_{G}^{*}\left(\bar{M}_{0,1}\left(\mathcal{Z}, \beta^{\prime}\right)\right)$ and note that the equivariant integral in equation (6.50) can be written as

$$
\int_{\left[\bar{M}_{P_{1}^{\prime}}\right]_{G}} \frac{e_{G}\left(\left[j_{P_{1}^{\prime}}^{*}\left(\mathcal{V}^{0}\right)-\Lambda^{4}\left(T_{\mathcal{Z}}\right)_{P_{1}^{\prime}}\right]\right)}{-\eta_{3} / d_{1}^{\prime}-\psi_{p}}=\frac{1}{e_{G}\left(\Lambda^{4}\left(T_{\mathcal{Z}}\right)_{P_{1}^{\prime}}\right)} \pi_{*} j_{P_{1}^{\prime}}^{*}(\alpha),
$$

where we use the notation of equation (6.26). Then, using the projection formula, we have

$$
\pi_{*} j_{P_{1}^{\prime}}^{*}(\alpha)=i_{P_{1}^{\prime}}^{!} e v_{*}\left(\alpha \cap\left[\bar{M}_{0,1}\left(\mathcal{Z}, \beta^{\prime}\right)\right]\right)
$$


All these manipulations are carried out in equivariant setting, but we suppressed the subscript $G$ for simplicity. If we denote by $\Pi: \mathcal{Z} \longrightarrow p t$ the projection onto a point, the equivariant integral in equation (6.51) can be written as

$$
\int_{\left[\bar{M}_{0,1}\left(\mathcal{Z}, \beta^{\prime}\right)\right]_{G}} \frac{e_{G}\left(\mathcal{V}^{0}\right) e v_{G}^{*}\left(\phi_{P_{1}^{\prime}}\right)}{-\eta_{3} / d_{1}^{\prime}-\psi_{p}}=\Pi_{*} e v_{*}\left(\alpha \cup e v^{*}\left(\phi_{P_{1}^{\prime}}\right) \cap\left[\bar{M}_{0,1}\left(\mathcal{Z}, \beta^{\prime}\right)\right]\right) .
$$

Now we can use localization on $\mathcal{Z}_{G}$ to rewrite the right-hand side of equation (6.54) as

$$
\begin{aligned}
& \Pi_{*} e v_{*}\left(\alpha \cup e v^{*}\left(\phi_{P_{1}^{\prime}}\right) \cap\left[\bar{M}_{0,1}\left(\mathcal{Z}, \beta^{\prime}\right)\right]\right) \\
& =\sum_{P_{\mathrm{f}}} i_{P_{\mathrm{f}}}^{!}\left(\phi_{P_{1}^{\prime}} \cap e v_{*}\left(\alpha \cup \cap\left[\bar{M}_{0,1}\left(\mathcal{Z}, \beta^{\prime}\right)\right]\right)\right) \cap \frac{1}{e_{G}\left(\left(T_{\mathcal{Z}}\right)_{P_{\mathrm{f}}}\right)} \\
& =\sum_{P_{\mathrm{f}}} \frac{i_{P_{\mathrm{f}}}^{*}\left(\phi_{P_{1}^{\prime}}\right)}{e_{G}\left(\left(T_{\mathcal{Z}}\right)_{P_{\mathrm{f}}}\right)} \cap i_{P_{\mathrm{f}}}^{!} e v_{*}\left(\alpha \cup \cap\left[\bar{M}_{0,1}\left(\mathcal{Z}, \beta^{\prime}\right)\right]\right),
\end{aligned}
$$

where the sum is over all fixed points $P_{\mathrm{f}}$ of the $G$ action of $\mathcal{Z}$. The equivariant Thom classes of the fixed points satisfy orthogonality conditions of the form

$$
i_{P_{\mathrm{f}}}^{*}\left(\phi_{P_{1}^{\prime}}\right)= \begin{cases}e_{G}\left(\left(T_{\mathcal{Z}}\right)_{P_{\mathrm{f}}}\right) & \text { if } P_{\mathrm{f}}=P_{1}^{\prime} \\ 0, & \text { if } P_{\mathrm{f}} \neq P_{1}^{\prime}\end{cases}
$$

Therefore, equation (6.55) reduces to

$$
\Pi_{*} e v_{*}\left(\alpha \cup e v^{*}\left(\phi_{P_{1}^{\prime}}\right) \cap\left[\bar{M}_{0,1}\left(\mathcal{Z}, \beta^{\prime}\right)\right]\right)=i_{P_{1}^{\prime}}^{!} e v_{*}\left(\alpha \cup \cap\left[\bar{M}_{0,1}\left(\mathcal{Z}, \beta^{\prime}\right)\right]\right)
$$

as claimed above.

\subsubsection{Horizontal discs}

Let us now consider horizontal discs, i.e., $f_{\Delta}$ is an invariant $d_{3}^{\prime}: 1$ cover of $D_{3}^{\prime}$. Most of the above considerations go through, but there is an important difference, namely the origin $Q_{3}$ of $D_{3}^{\prime}$ is not a fixed point under the generic $G$ action. Instead, it is a degenerate $T$-fixed point lying on the fixed curve $\overline{P_{3} P_{3}^{\prime}}$. The contribution of the isolated fixed point $\left(\Delta, f_{\Delta}\right)$ can be computed along the same lines

$$
\mathcal{C}_{1,3^{\prime}}\left(d_{3}^{\prime}, d_{3}^{\prime} h_{2}\right)=\frac{(-1)^{d_{3}^{\prime}}}{d_{3}^{\prime 3-d_{3}^{\prime}}} \frac{\prod_{n=1}^{d_{3}^{\prime}-1}\left(\lambda_{1}-\left(n / d_{3}^{\prime}\right) \lambda_{3}\right)}{\lambda_{3}^{d_{3}^{\prime}-1}} .
$$

The overall sign is again ambiguous; the present choice should be regarded as part of the prescriptions of the duality map. Formula (6.46) goes through 
essentially unchanged

$$
\mathcal{C}_{1,3^{\prime}}\left(d_{3}^{\prime}, \beta\right)=\frac{\mathcal{C}_{1,3^{\prime}}\left(d_{3}^{\prime}, d_{3}^{\prime} h_{2}\right)}{\left(-\lambda_{t} H\right)} \int_{\left[\bar{M}_{Q_{3}}\right]_{T}} \frac{e_{T}\left(\left[j_{Q_{3}}^{*}\left(\mathcal{V}^{0}\right)-\Lambda^{4}\left(T_{\mathcal{Z}}\right)_{Q_{3}}\right]\right)}{-\lambda_{t} H-\psi_{p}},
$$

where $\lambda_{t}=\lambda_{3} / d_{3}^{\prime}$ is the weight of the induced torus action on the domain. Now we have to integrate against the virtual cycle of the moduli space $\bar{M}_{Q_{3}}$ defined by the following diagram

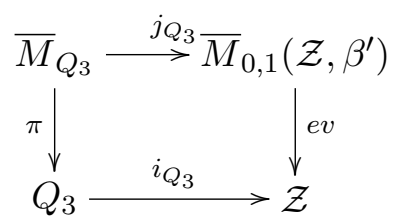

and $\beta^{\prime}=\beta-d_{3}^{\prime} h_{2}$.

At this point, we encounter an extra complication, since $Q_{3}$ is not invariant under the generic torus action. Nevertheless, we would still like to rewrite equation (6.58) in terms of a generic torus action in order to perform the computations efficiently. A key observation is that there exists a $T$-equivariant automorphism $h: \mathcal{Z} \longrightarrow \mathcal{Z}$ mapping the $G$-fixed point $P_{3}^{\prime}=$ $\left\{Z_{1}=Z_{4}=U=W=0\right\}$ to $Q_{3}$. In terms of homogeneous coordinates, $h$ is given by

$$
h:\left[Z_{1}, Z_{2}, Z_{3}, Z_{4}, U, V\right] \longrightarrow\left[Z_{1}, Z_{2}, Z_{3}, Z_{4}, U, V, W-\mu V_{2} V_{3}\right] .
$$

It is straightforward to check that equation (6.61) is compatible with the $\left(\mathbb{C}^{*}\right)^{3}$ action $(6.1)$ and the $T$-action on $\mathcal{Z}$. Let $\bar{M}_{P_{3}^{\prime}}$ be the subspace of the moduli space $\bar{M}_{0,1}\left(\mathcal{Z}, \beta^{\prime}\right)$ defined by a commutative diagram of the form (6.60), with $Q_{3}$ replaced by $P_{3}^{\prime}$. Since $P_{3}^{\prime}$ is a fixed point, there is an induced $T$-action on $\bar{M}_{P_{3}^{\prime}}$. Then composition by $h: \mathcal{Z} \longrightarrow \mathcal{Z}$ induces a $T$-equivariant automorphism $\Psi_{h}: \bar{M}_{0,1}\left(\mathcal{Z}, \beta^{\prime}\right) \longrightarrow \bar{M}_{0,1}\left(\mathcal{Z}, \beta^{\prime}\right)$ and we obtain a commutative diagram of the form

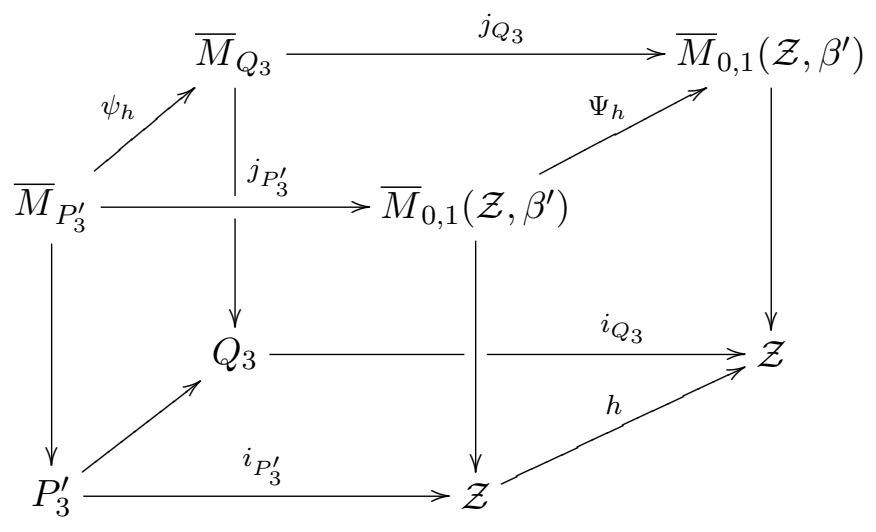


The obstruction bundle $\mathcal{V}_{0}$ over $\bar{M}_{0,1}\left(\mathcal{Z}, \beta^{\prime}\right)$ and the virtual cycle $\left[\bar{M}_{0,1}\right.$ $\left.\left(\mathcal{Z}, \beta^{\prime}\right)\right]$ are invariant under $h$ by construction. That is, we have $\Psi_{h}^{*}\left(\mathcal{V}_{0}\right)=$ $\mathcal{V}_{0}$ and $h^{!}\left[\bar{M}_{0,1}\left(\mathcal{Z}, \beta^{\prime}\right)\right]=\left[\bar{M}_{0,1}\left(\mathcal{Z}, \beta^{\prime}\right)\right]$. The virtual cycles $\left[\bar{M}_{P_{3}^{\prime}}\right]$ and $\left[\bar{M}_{Q_{3}}\right]$ are determined by Gysin maps $\left[\bar{M}_{P_{3}^{\prime}}\right]=i_{P_{3}^{\prime}}^{!}\left[\bar{M}_{0,1}\left(\mathcal{Z}, \beta^{\prime}\right)\right]$ and $\left[\bar{M}_{Q_{3}}\right]=i_{Q_{3}}^{!}$ $\left[\bar{M}_{0,1}\left(\mathcal{Z}, \beta^{\prime}\right)\right]$. Therefore, we have $\psi_{h}^{!}\left[\bar{M}_{Q_{3}}\right]=\left[\bar{M}_{P_{3}^{\prime}}\right]$ and $\psi_{h}^{*} j_{Q_{3}}^{*}\left(\mathcal{V}_{0}\right)=j_{P_{3}^{\prime}}^{*}$ $\left(\mathcal{V}_{0}\right)$. Moreover, $\Lambda^{4}\left(T_{\mathcal{Z}}\right)_{Q_{3}} \simeq \Lambda^{4}\left(T_{\mathcal{Z}}\right)_{P_{3}^{\prime}}$ as $T$-vector spaces. We conclude that the equivariant integral (6.59) is equal to

$$
\mathcal{C}_{1,3^{\prime}}\left(d_{3}^{\prime}, \beta\right)=\frac{\mathcal{C}_{1,3^{\prime}}\left(d_{3}^{\prime}, d_{3}^{\prime} h_{2}\right)}{\left(-\lambda_{t} H\right)} \int_{\left[\bar{M}_{P_{3}^{\prime}}\right]_{T}} \frac{e_{T}\left(\left[j_{P_{3}^{\prime}}^{*}\left(\mathcal{V}^{0}\right)-\Lambda^{4}\left(T_{\mathcal{Z}}\right)_{P_{3}^{\prime}}\right]\right)}{-\lambda_{t} H-\psi_{p}} .
$$

Since $P_{3}^{\prime}$ is fixed under the generic torus action, we can further rewrite this expression as

$$
\mathcal{C}_{1,3^{\prime}}\left(d_{3}^{\prime}, \beta\right)=\frac{\mathcal{C}_{1,3^{\prime}}\left(d_{3}^{\prime}, d_{3}^{\prime} h_{2}\right)}{\left(-\lambda_{t} H\right)} s^{*}\left[\int_{\left[\bar{M}_{P_{3}^{\prime}}\right]_{G}} \frac{e_{T}\left(\left[j_{P_{3}^{\prime}}^{*}\left(\mathcal{V}^{0}\right)-\Lambda^{4}\left(T_{\mathcal{Z}}\right)_{P_{3}^{\prime}}\right]\right)}{\eta_{3} / d_{3}^{\prime}-\psi_{p}}\right],
$$

or, equivalently, as

$$
\begin{aligned}
\mathcal{C}_{1,3^{\prime}}\left(d_{1}^{\prime}, \beta\right)=\frac{\mathcal{C}_{1,3^{\prime}}\left(d_{3}^{\prime}, d_{3}^{\prime} h_{2}\right)}{\left(-\lambda_{t} H\right)} s^{*} \\
\quad \times\left[\frac{1}{e_{G}\left(\Lambda^{4}\left(T_{\mathcal{Z}}\right)_{P_{3}^{\prime}}\right)} \int_{\left[\bar{M}_{0,1}\left(\mathcal{Z}, \beta^{\prime}\right)\right]_{G}} \frac{e_{G}\left(\mathcal{V}^{0}\right) e v^{*}\left(\phi_{P_{3}^{\prime}}\right)}{\eta_{3} / d_{3}^{\prime}-\psi_{p}}\right] .
\end{aligned}
$$

This formula will be used for explicit computations in the next section.

\subsubsection{Multiple boundary components}

We are left with open string maps with several boundary components. Let $f: \Sigma \longrightarrow \mathcal{Z}, \Sigma=\Sigma_{0} \cup \Delta_{1} \cup \cdots \cup \Delta_{h}$ be such a map of type $\left(\rho, d_{a}\right)$. Recall that $\rho:\{1, \ldots, h\} \longrightarrow\left\{1^{\prime}, \ldots, 4^{\prime}, 1^{\prime \prime}, \ldots, 4^{\prime \prime}\right\}$ specifies the image of each disc $\Delta_{a}, a=1, \ldots, h$, of the domain, and $d_{a}$ are the corresponding degrees.

If $\Sigma_{0}$ is nonempty, we proceed by analogy with the previous two cases. A similar sequence of arguments shows that the sum over all open string local contributions can be written as a moduli space integral of the form

$$
\begin{aligned}
\mathcal{C}_{h, \rho}\left(d_{a}, \beta\right)= & \frac{1}{|\mathcal{P}|} \prod_{a=1}^{h} \frac{\mathcal{C}_{1, \rho(a)}\left(d_{a}, d_{a}\left[D_{\rho(a)}\right]\right)}{-\left(\lambda_{t_{a}} H\right)} s^{*}\left[\frac{1}{\prod_{a=1}^{h} e_{G}\left(\Lambda^{4}\left(T_{\mathcal{Z}}\right)_{P(a)}\right)}\right. \\
& \left.\times \int_{\left[\bar{M}_{0, h}\left(\mathcal{Z}, \beta^{\prime}\right)\right]_{G}} e_{G}\left(\mathcal{V}^{0}\right) \prod_{a=1}^{h} \frac{e v_{a}^{*}\left(\phi_{P(a)}\right)}{-\kappa_{a}-\psi_{p_{a}}}\right]
\end{aligned}
$$


Let us explain the notation. The coefficient $\mathcal{C}_{1, \rho(a)}\left(d_{a}, d_{a}\left[D_{\rho(a)}\right]\right)$ represents the multicover contribution of the ath disc of the domain which is mapped $d_{a}: 1$ to the disc $D_{\rho(a)}$ in the target space. $P$ is a function from the index set $\{1, \ldots, h\}$ to the set of fixed points of $\mathcal{Z}$ under the $G$ action. If $D_{\rho(a)}$ is a vertical disc, $P(a)$ is the origin of $D_{\rho(a)}$; if $D_{\rho(a)}$ is horizontal, then we have $P(a)=P_{3}^{\prime}$ if $\rho(a)=3^{\prime}, 4^{\prime}$, and $P(a)=P_{4}^{\prime \prime}$ if $\rho(a)=$ $3^{\prime \prime}, 4^{\prime \prime} . \bar{M}_{0, h}\left(\mathcal{Z}, \beta^{\prime}\right)$ is the moduli space of stable closed string maps to $\mathcal{Z}$ with $h$ marked points $\left\{p_{a}\right\}$. The homology class $\beta^{\prime} \in H_{2}(\mathcal{Z}, \mathbb{Z})$ is given by $\beta^{\prime}=\beta-\sum_{a=1}^{h}\left[D_{\rho(a)}\right]$. ev: $\bar{M}_{0, h}\left(\mathcal{Z}, \beta^{\prime}\right) \longrightarrow \mathcal{Z}^{h}$ is the evaluation map and $e v_{a}: \bar{M}_{0, h}\left(\mathcal{Z}, \beta^{\prime}\right) \longrightarrow \mathcal{Z}$ denotes its $a$ th component. $\kappa_{a}, a=1, \ldots, h$, is an equivariant class in $\mathcal{K}_{G}$ such that $s^{*}\left(\kappa_{a}\right)=\lambda_{t_{a}} H$, where $\lambda_{t_{a}}$ is the weight of the induced torus action on the disc $\Delta_{a}$. One can easily check that $\kappa_{a}=$ $\eta_{3} / d_{a}$ if $\rho(a)=1^{\prime}, 3^{\prime \prime}, \kappa_{a}=\eta_{1} / d_{a}$ if $\rho(a)=1^{\prime \prime}, 4^{\prime}, \kappa_{a}=-\eta_{3} / d_{a}$ if $\rho(a)=2^{\prime \prime}, 3^{\prime}$ and $\kappa_{a}=-\eta_{1} / d_{a}$ if $\rho(a)=2^{\prime}, 4^{\prime \prime}$. Finally, $1 /|\mathcal{P}|$ is a symmetry factor which takes into account the automorphism group of a fixed locus. The automorphism group of an invariant open string map as above is of the form $\prod_{a=1}^{h}\left(\mathbb{Z} / d_{a} \mathbb{Z}\right) \times \mathcal{P}$. The first $h$ factors represent deck transformations of the Galois cover $f_{\Delta_{a}}: \Delta_{a} \longrightarrow D_{\rho(a)}$. Their effect would be a prefactor $\prod_{a=1}^{h} 1 / d_{a}$ in equation (6.66) which is absorbed in the coefficients $\mathcal{C}_{1, \rho(a)}\left(d_{a}, d_{a}\left[D_{\rho(a)}\right]\right)$. $\mathcal{P}$ is a subgroup of the permutation group $\mathcal{S}_{h}$ which permutes the marked points $p_{a}$ leaving the map $d_{a}$ unchanged. More precisely, if we have $n$ discs of the domain mapping to the same disc in $\mathcal{Z}, \mathcal{P}$ contains a factor $\mathcal{S}_{n}$. Therefore, we obtain a factor $1 / n$ ! in equation (6.66) for each such group of $n$ discs. There are eight discs in the target space, so we can have up to eight factors of this form.

If $\Sigma_{0}$ is empty, the domain of $f$ can be either a single disc or a two discs with common origin forming a nodal cylinder. The first case has been treated above. A map of the form $f: \Delta_{1} \longrightarrow \mathcal{Z}$ contributes $\mathcal{C}_{1, \rho(1)}\left(d_{1}, d_{1}\left[D_{\rho(1)}\right]\right)$ to the instanton expansion. The second case follows easily from the first using normalization exact sequences. Given a map $f: \Delta_{1} \cup_{p} \Delta_{2} \longrightarrow \mathcal{Z}$ of type $\left(\rho, d_{1}, d_{2}\right)$ as above, its contribution can be easily shown to be of the form

$$
\begin{aligned}
\mathcal{C}_{1,2, \rho(1), \rho(2)}\left(d_{1}, d_{2}, d_{1}\left[D_{\rho(1)}\right]+d_{2}\left[D_{\rho(2)}\right]\right) \\
=\mathcal{C}_{1, \rho(1)}\left(d_{1}, d_{1}\left[D_{\rho(1)}\right]\right) \mathcal{C}_{1, \rho(2)}\left(d_{2}, d_{2}\left[D_{\rho(2)}\right]\right) \\
\quad \times \frac{e_{T}\left(\left[\left(T_{\mathcal{Z}}\right)_{P(1)}-\Lambda^{4}\left(T_{\mathcal{Z}}\right)_{P(1)}\right]\right)}{\left(-\lambda_{t_{1}} H\right)\left(-\lambda_{t_{2}} H\right)\left(-\lambda_{t_{1}}-\lambda_{t_{2}}\right) H} .
\end{aligned}
$$

For a uniform treatment, we can extend the formula (6.66) to encompass all possible cases. By convention, if $\Sigma_{0}$ is empty, equation (6.66) should be interpreted as explained in the current paragraph. 
This concludes our discussion of open string instantons on compact threefolds. Since this section is rather long and complicated, let us summarize the main points. The open string instanton corrections take the form (6.21), where the enumerative invariants $\mathcal{C}_{h, \rho}\left(d_{a}, \beta\right)$ are given by equation (6.66), which is our main formula. Although this expression has been derived starting from heuristic considerations, it is a well-defined equivariant integral over a moduli space (or stack) of stable maps. In the next section, we will find very convincing evidence for the approach proposed here by direct computations and comparison with the closed string dual.

\section{Explicit computations and large $N$ duality}

At this stage, we have all the ingredients needed for computing topological open string amplitudes and testing large $N$ duality. Some preliminary remarks on large $N$ duality for compact three-folds have been included in Section 2. Let us start with a more precise account of the duality predictions for the extremal transition under consideration.

\subsection{Large $N$ duality predictions}

As explained in Section 4, we have an extremal transition between smooth projective Calabi-Yau three-folds $Y, \widetilde{Y}$ which can be represented as hypersurfaces in toric varieties $\mathcal{Z}, \widetilde{\mathcal{Z}}$. $\widetilde{\mathcal{Z}}$ is the blow-up of $\mathcal{Z}$ along the section $U=V=0$ and $\tilde{Y}$ the strict transform of the singular hypersurface $Y_{0}$. $\widetilde{\mathcal{Z}}$ has the following toric presentation

$\begin{array}{lcccccccc} & \widetilde{Z}_{1} & \widetilde{Z}_{2} & \widetilde{Z}_{3} & \widetilde{Z}_{4} & \widetilde{U} & \widetilde{V} & \widetilde{W} & \widetilde{T} \\ \mathbb{C}^{*} & 1 & 1 & 0 & 0 & -1 & -1 & 0 & 0 \\ \mathbb{C}^{*} & 0 & 0 & 1 & 1 & -1 & -1 & 0 & 0 \\ \mathbb{C}^{*} & 0 & 0 & 0 & 0 & 1 & 1 & 1 & 0 \\ \mathbb{C}^{*} & 0 & 0 & 0 & 0 & 1 & 1 & 0 & -1\end{array}$

One can easily check that $h^{1,1}(\widetilde{Z})=h^{1,1}(Z)+1$, therefore $r=1$ in the notation of Section 2. The toric contraction $\pi: \widetilde{\mathcal{Z}} \longrightarrow \mathcal{Z}$ is given in terms of homogeneous coordinates by

$$
Z_{i}=\widetilde{Z}_{i}, \quad i=1, \ldots, 4, \quad U=\widetilde{U} \widetilde{T}, \quad V=\widetilde{V} \widetilde{T}, \quad W=\widetilde{W}
$$

with exceptional divisor $(\widetilde{T})$. Note that $(\widetilde{T})$ is isomorphic to $\mathbb{P}^{1} \times \mathbb{F}_{0}$ and the map (7.2) contracts the $\mathbb{P}^{1}$ fibers. 
Geometrically, $\widetilde{\mathcal{Z}}$ is a fibration over $\mathbb{F}_{0}$ with $\mathbb{F}_{1}$ fibers. This follows from the fact that each $\mathbb{P}^{2}$ fiber of $\mathcal{Z} \longrightarrow \mathbb{F}_{0}$ undergoes an embedded one-point blow-up. The Mori cone of $\widetilde{\mathcal{Z}}$ is generated by (see equation (A.1))

$$
\begin{array}{llll}
\widetilde{h}_{1}: & \widetilde{Z}_{1}=\widetilde{Z}_{3}=\widetilde{T}=0, & \widetilde{h}_{2}: & \widetilde{Z}_{1}=\widetilde{U}=\widetilde{T}=0, \\
\widetilde{h}_{3}: & \widetilde{Z}_{3}=\widetilde{U}=\widetilde{T}=0, & \widetilde{h}_{4}: & \widetilde{Z}_{1}=\widetilde{Z}_{3}=\widetilde{U}=0 .
\end{array}
$$

Note that $\widetilde{h}_{1}$ and $\widetilde{h}_{4}$ are vertical classes and $\widetilde{h}_{2}$ and $\widetilde{h}_{3}$ are horizontal classes on $\widetilde{\mathcal{Z}}$. Moreover, $\widetilde{h}_{1}$ is the fiber class of $(\widetilde{T})$, and $\widetilde{h}_{1} \cdot(\widetilde{T})=-1$. Therefore in the notation of Section 2 , we have a single exceptional curve class $[C]=\widetilde{h}_{1}$, and one exceptional divisor $D=-(\widetilde{T})$ so that $C \cdot D=1$.

The Kähler cones of $\mathcal{Z}$ and $\widetilde{\mathcal{Z}}$ are generated by the toric divisors (see formulae (A.1) and (A.3) in Appendix A)

$$
\begin{array}{llll}
J_{1} & =(W), & J_{2}=\left(Z_{1}\right), & J_{3}=\left(Z_{3}\right) \\
\widetilde{J}_{1}=(\widetilde{U}), & \widetilde{J}_{2}=\left(\widetilde{Z}_{1}\right), & \widetilde{J}_{3}=\left(\widetilde{Z}_{3}\right), & \widetilde{J}_{4}=(\widetilde{W}) .
\end{array}
$$

Using equation (7.2) and standard linear relations among toric divisors, we find the following relations

$$
\widetilde{J}_{1}=\pi^{*}\left(J_{1}-J_{2}-J_{3}\right)+(\widetilde{T}), \quad \widetilde{J}_{2}=\pi^{*}\left(J_{2}\right), \quad \widetilde{J}_{3}=\pi^{*}\left(J_{3}\right), \quad \widetilde{J}_{4}=\pi^{*}\left(J_{1}\right) .
$$

Therefore, the geometric part of the duality map (2.9) becomes in this case

$$
\tilde{t}_{1}=-\mathrm{i} \lambda, \quad \widetilde{t}_{2}-\widetilde{t}_{1}=t_{2}, \quad \widetilde{t}_{3}-\widetilde{t}_{1}=t_{3}, \quad \widetilde{t}_{4}+\widetilde{t}_{1}=t_{1} .
$$

In order to test the duality predictions, equation (7.6) must be supplemented with extra relations between the open string Kähler parameters $\tau_{i}^{\prime}$ and $\tau_{i}^{\prime \prime}$, $i=1, \ldots, 4$, and the closed string parameters $\widetilde{t}_{\gamma}, \gamma=1, \ldots, 4$. These will be determined later. We have to compare the genus zero Gromov-Witten expansion of $\widetilde{Y}$

$$
\mathcal{F}_{\widetilde{Y} ; \mathrm{cl}}^{(0)}\left(g_{s}, \widetilde{t}_{\gamma}\right)=g_{s}^{-2} \sum_{\widetilde{\beta} \in H_{2}(\widetilde{\mathcal{Z}, \mathbb{Z})}} \widetilde{C}_{\widetilde{\beta}} \mathrm{e}^{-\langle\widetilde{J}, \widetilde{\beta}\rangle}
$$

to the genus zero open string expansion of $(Y, L)$, or, more precisely, to a generating functional attached to the triple $(\mathcal{Z}, \bar{Y}, \bar{L})$.

Since the discussion in Section 2 was very schematic, let us summarize the main points of the construction. We start with a generating functional 
of the form

$$
\begin{aligned}
& F_{\text {inst }}\left(g_{\mathrm{s}}, t_{\alpha}, \tau_{i}^{\prime}, \tau_{i}^{\prime \prime}\right) \\
& \quad=\sum_{h=1}^{\infty} \sum_{\left(d_{a}, \beta\right)} \sum_{\rho} C_{h, \rho}\left(d_{a}, \beta\right) \mathrm{e}^{-\left\langle J, \beta-\sum_{a=1}^{h} d_{a}\left[D_{\rho(a)}\right]\right\rangle} \mathrm{e}^{-\sum_{a=1}^{h} d_{a} \tau_{\rho(a)}} \prod_{a=1}^{h} \operatorname{Tr} V_{\rho(a)}^{d_{a}},
\end{aligned}
$$

where the notation has been explained in detail in Section 6 , and will not be reviewed here. The coefficients $C_{h, \rho}\left(d_{a}, \beta\right)=i_{p t}^{*} \mathcal{C}_{h, \rho}\left(d_{a}, \beta\right)$ are defined by our main formula (6.66), which is reproduced below for convenience

$$
\begin{aligned}
\mathcal{C}_{h, \rho}\left(d_{a}, \beta\right)= & \frac{1}{|\mathcal{P}|} \prod_{a=1}^{h} \frac{\mathcal{C}_{1, \rho(a)}\left(d_{a}, d_{a}\left[D_{\rho(a)}\right]\right)}{-\lambda_{t_{a}} H} s^{*}\left[\frac{1}{\prod_{a=1}^{h} e_{G}\left(\Lambda^{4}\left(T_{\mathcal{Z}}\right)_{P(a)}\right)}\right. \\
& \left.\times \int_{\left[\bar{M}_{0, h}\left(\mathcal{Z}, \beta^{\prime}\right)\right]_{G}} e_{G}\left(\mathcal{V}^{0}\right) \prod_{a=1}^{h} \frac{e v_{a}^{*}\left(\phi_{P(a)}\right)}{-\kappa_{a}-\psi_{p_{a}}}\right] .
\end{aligned}
$$

The formal series (7.8) is called the open string instanton sum and should be interpreted as a series of corrections to Chern-Simons theory. ${ }^{9}$ The final open string generating functional is the Chern-Simons free energy with all these corrections taken into account. Therefore, the final expression for the genus zero free energy will be of the form

$$
\begin{aligned}
\mathcal{F}_{(Y, L) ; \mathrm{op}}^{(0)}\left(g_{\mathrm{s}}, t_{\alpha}, \lambda, \tau_{i}^{\prime}, \tau_{i}^{\prime \prime}\right)= & \mathcal{F}_{Y ; \mathrm{cl}}^{(0)}\left(g_{\mathrm{s}}, t_{\alpha}\right)+\mathcal{F}_{\mathrm{CS}, 1}^{(0)}+\mathcal{F}_{\mathrm{CS}, 2}^{(0)} \\
& +\sum_{d_{i}^{\prime}, d_{i}^{\prime \prime}, \beta^{\prime}} F_{d_{i}^{\prime}, d_{i}^{\prime \prime}, \beta}\left(g_{\mathrm{s}}, \lambda\right) \mathrm{e}^{-\left\langle J, \beta^{\prime}\right\rangle} \mathrm{e}^{-\sum_{i=1}^{4}\left(d_{i}^{\prime} \tau_{i}^{\prime}+d_{i}^{\prime \prime} \tau_{i}^{\prime \prime}\right)},
\end{aligned}
$$

where

$$
\mathcal{F}_{Y ; \mathrm{cl}}^{(0)}=g_{\mathrm{s}}^{-2} \sum_{\beta \in H_{2}(\mathcal{Z}, \mathbb{Z})} C_{\beta} \mathrm{e}^{-\langle J, \beta\rangle}
$$

is the genus zero Gromov-Witten expansion of $Y$. The next two terms in equation (7.10) represent the genus zero contributions of the uncorrected Chern-Simons theories supported on $\bar{L}_{1}$ and $\bar{L}_{2}$ and the last sum encodes the effect of open string instantons. Note that we sum over all relative homology classes $\beta=\beta^{\prime}+\sum_{i=1}^{4}\left(d_{i}^{\prime}\left[D_{i}^{\prime}\right]+d_{i}^{\prime \prime}\left[D_{i}^{\prime \prime}\right]\right)$, with $d_{i}^{\prime}, d_{i}^{\prime \prime} \geq 0$. In the remaining part of this section, we will show by explicit computations that equations (7.7) and (7.10) are in exact agreement. This is very strong evidence for the large $N$ duality, as well as the open string techniques developed here.

Let us start with some remarks on the instanton coefficients (7.9). This formula factorizes in open and closed string contributions reflecting the

\footnotetext{
${ }^{9}$ Note that it suffices to include only genus zero corrections in this formula, in agreement with the footnote at page 24 .
} 
structure of a generic invariant open string map. The open string contributions are represented by the prefactor $\prod_{a=1}^{h} \mathcal{C}_{1, \rho(a)}\left(d_{a}, d_{a}\left[D_{\rho(a)}\right]\right) /-\left(\lambda_{t_{a}} H\right)$ while the closed string contribution is the equivariant integral in square brackets, which takes values in $\mathcal{K}_{G} \simeq \mathbb{Q}\left(\eta_{1}, \ldots, \eta_{7}\right)$. This integral can be evaluated by localization with respect to the $G$-action on the moduli space $\bar{M}_{0, h}\left(\mathcal{Z}, \beta^{\prime}\right)$. The fixed loci of this action can be classified using the graph method developed in ref. [36] and the evaluation of local contributions is standard material. The open string factors can be represented graphically by adding extra legs to the closed string graphs as in ref. [25]. There is, however, a subtlety in this approach, as the closed string graphs represent fixed loci under the induced $G$-action on the moduli space of marked stable maps. On the other hand, $G$ does not act on the moduli space of open string maps, as explained in detail in Section 6. Hence a closed string graph with extra legs should not be interpreted naively as a graphical representation of an open string fixed locus. Instead, one should think of such a graph as encompassing two sets of data corresponding to the factorization of equation (7.9) into open and closed string contributions. The closed string data are encoded in a conventional closed string graph, while the open string data is encoded in the extra legs. We will shortly discuss concrete examples.

The homomorphism $s^{*}: \mathcal{K}_{G} \longrightarrow \mathcal{K}_{T}$ has been described in detail in Section 6 , below equation (6.49). We have $s^{*}\left(\eta_{1,3}\right)=\lambda_{1,3} H, s^{*}\left(\eta_{2,4,7}\right)=s^{*}\left(\eta_{1}+\right.$ $\left.\eta_{5}\right)=s^{*}\left(\eta_{3}+\eta_{6}\right)=0$. These formulae must be extended to $\mathcal{K}_{G}$ by localization. Since we also have to take the nonequivariant limit of equation (7.9), the final answer will be a homogeneous rational function of $\left(\lambda_{1}, \lambda_{3}\right)$ of degree zero. It is very convenient to express the answer as a rational function of the ratio $z=\lambda_{3} / \lambda_{1}$. Following the local examples discussed in Section 5, $z$ will be related to framing in Chern-Simons theory, provided that the later can be thought of as a formal variable. We will show that this algorithm gives rise to sensible results, although we will find some subtleties along the way.

Spheres $\bar{L}_{1}$ and $\bar{L}_{2}$ contain two knots $\Gamma_{1}^{\prime}$ and $\Gamma_{1}^{\prime \prime}$ and, respectively, $\Gamma_{2}^{\prime}$ and $\Gamma_{2}^{\prime \prime}$ which form Hopf links with linking number +1 . As explained in the paragraph below equation (6.21), these knots are endowed with the orientation induced by the canonical orientation of the vertical discs with respect to the inner normal convention. This means that the multicover contributions of the vertical discs will be weighted by holonomy factors of the form $\operatorname{Tr}\left(V_{1}^{\prime}\right)^{d}$ and $\operatorname{Tr}\left(V_{1}^{\prime \prime}\right)^{d}$ and, respectively, $\operatorname{Tr}\left(V_{2}^{\prime}\right)^{d}$ and $\operatorname{Tr}\left(V_{2}^{\prime \prime}\right)^{d}$, where $d>0$ is the degree. The contributions of the horizontal discs will be weighted by holonomy factors of the form $\operatorname{Tr}\left(V_{1}^{\prime}\right)^{-d}=\operatorname{Tr}\left(\overline{V_{1}^{\prime}}\right)^{d}$, etc. ${ }^{10}$ The framing of $\Gamma_{1}^{\prime}, \ldots, \Gamma_{2}^{\prime \prime}$ can be related to the torus weights following the same

\footnotetext{
${ }^{10}$ Here we denote by convention $\operatorname{Tr}_{R} \bar{V}=\operatorname{Tr}_{\bar{R}} V$ for any $U(N)$ group element $V$.
} 
considerations as in the local case. We find the following relations

$$
p_{1}^{\prime}=\frac{1}{z}, \quad p_{1}^{\prime \prime}=z, \quad p_{2}^{\prime}=z, \quad p_{2}^{\prime \prime}=\frac{1}{z} .
$$

After these preliminary remarks, let us turn to concrete computations. For a systematic approach, we will distinguish several cases, depending on the class $\beta \in H_{2}(\mathcal{Z}, \mathbb{Z})$.

\subsection{Vertical classes}

We start with instanton corrections associated to vertical homology classes. This means that the discs $\Delta_{a}$ are mapped to the vertical discs $D_{1}^{\prime}, D_{1}^{\prime \prime}, D_{2}^{\prime}$, $D_{2}^{\prime \prime}$, and the class $\beta^{\prime}$ associated to the closed curve $\Sigma_{0}$ is a multiple of $h_{1}$. Let us denote by $n$ the total degree of such a map, i.e., $\beta=n h_{1}, n \geq 0$. The class $\beta^{\prime}$ introduced in Section 6 will be of the form $\beta^{\prime}=n^{\prime} h_{1}$, with $0 \leq n^{\prime}<n$. For a fixed $n$, we have to sum over all values of $n^{\prime}$. Except for the individual disc factors in equation (6.66) the computation reduces to the evaluation of the equivariant integral by localization. The fixed loci in the moduli space of stable maps with marked points consist of points of the form $\left(\Sigma_{0}, f_{0}, p_{a}\right)$ where the marked points $p_{a}$ are mapped to $\left\{P_{1}^{\prime}, P_{1}^{\prime \prime}, P_{2}^{\prime}, P_{2}^{\prime \prime}\right\}$ and $f_{0}\left(\Sigma_{0}\right)$ is a $G$-invariant vertical curve on $\mathcal{Z}$. The structure of the $G$-fixed locus on $\mathcal{Z}$ and the $G$-invariant curves have been described in detail in Section 6.1. There we found a "skeleton" consisting of 24 invariant curves which is reproduced below for convenience.

The vertical invariant curves form four connected (reducible) components lying in four distinct $\mathbb{P}^{2}$ fibers which were denoted by $F_{1}, \ldots, F_{4}$. These are the green triangles in figure 8 . Since the marked points must be mapped to either $\left\{P_{1}^{\prime}, P_{1}^{\prime \prime}\right\}$ or $\left\{P_{2}^{\prime}, P_{2}^{\prime \prime}\right\}$, it follows that $\Sigma_{0}$ can be mapped either to
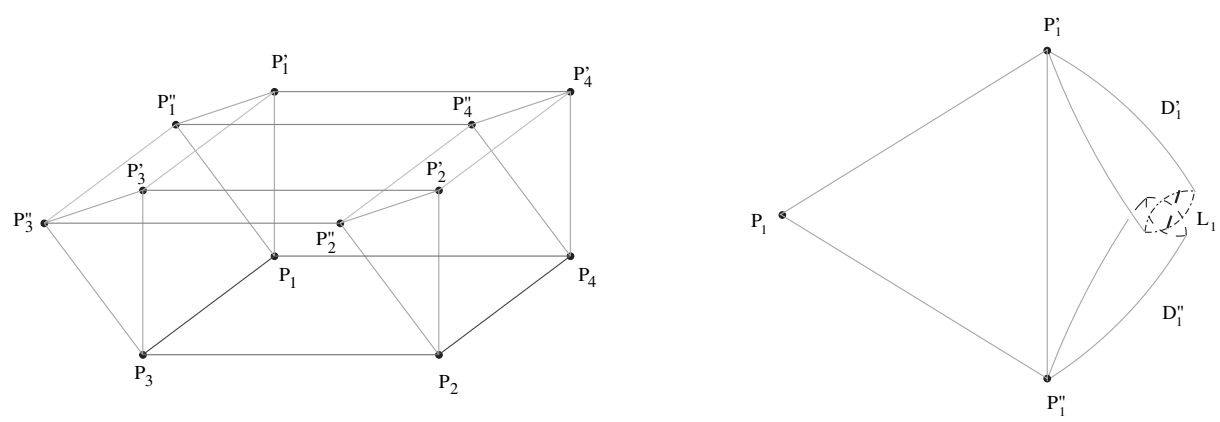

Figure 8: The toric skeleton of $\mathcal{Z}$ and the configuration of vertical discs attached to the fiber $F_{1}$. The color coding has been explained in Section 6 . 
$F_{1}$ or to $F_{2}$, which are disjoint fibers. Therefore, the fixed loci naturally divided into two classes depending on their image. Moreover, there is a $\mathbb{Z} / 2$ symmetry which exchanges the fixed loci in different classes. For each fixed locus mapping to $F_{1}$, there is an identical fixed locus mapping to $F_{2}$. A straightforward local computation shows that their contributions are also identical, and therefore it suffices to consider only one class, say $G$-invariant maps to $F_{1}$. The local geometry near $F_{1}$ is sketched in figure 8 . The fixed loci are represented by closed string graphs comprised continuous line segments. Additionally, we have extra legs corresponding to the open string factors, as explained below equation (6.48). In particular, we can have pure open string graphs when the domain of the map $f$ consists only of disc components.

The image of each irreducible component of the domain is specified by the inclination angle of the corresponding line segment and by the color code. Uncontracted components are represented by green lines with marked endpoints. Contracted components are represented by black line segments with no marking at the endpoints. The degree of the map onto its image is specified by a number $d$ attached to each segment unless $d=0,1$ in which case the segment is left unlabeled. For example, the graph (f3) in figure 9 represents an invariant map $f: \mathbb{P}^{1} \longrightarrow \mathcal{Z}$ which is mapped 1: 1 to $\overline{P_{1}^{\prime} P_{1}^{\prime \prime}}$. The marked point $p_{1}$ is mapped to $P_{1}^{\prime}$. This corresponds to a certain local contribution to the equivariant integral

$$
s^{*}\left[\frac{1}{e_{G}\left(\Lambda^{4}\left(T_{\mathcal{Z}}\right)_{P(1)}\right)} \int_{\left[\bar{M}_{0, h}\left(\mathcal{Z}, h_{1}\right)\right]_{G}} e_{G}\left(\mathcal{V}^{0}\right) \frac{e v_{1}^{*}\left(\phi_{P(1)}\right)}{-\eta_{3}-\psi_{p_{1}}}\right] .
$$

In addition, we have an open string prefactor of the form $C_{1,1^{\prime}}\left(1,\left[D_{1}^{\prime}\right]\right)$ corresponding to the dashed line segment. According to the discussion below equation (6.48), this should not be interpreted as graphical representation corresponding to a fixed invariant map. The correct point of view is to regard this graphs as a pair (closed string graph, open string graph) representing a local contribution to the instanton coefficient $C_{1,1^{\prime}}\left(1,2 h_{1}\right)$ defined in equation (7.9). Similarly, (f5) consists of a closed string graph which represents a $f: \mathbb{P}^{1} \longrightarrow \mathcal{Z}$ which is $1: 1$ onto $\overline{P_{1} P_{1}^{\prime}}$. The marked point $p_{1}$ is mapped to $P_{1}^{\prime}$. The extra dashed leg represents an open string factor given by a degree 1 cover of $D_{1}^{\prime}$. The graph (f15) has a similar interpretation, except that the domain of the closed string map has three irreducible components. One component is contracted (the black line) and two other components are are mapped to $\overline{P_{1}^{\prime} P_{1}^{\prime \prime}}$ with degree 1 . The open string data consist again of a degree 1 cover of $D_{1}^{\prime}$.

We list below all contributions of vertical maps to $F_{1}$ of total degree up to 3. Each local contribution carries a subscript which corresponds to a fixed locus represented in figures 9-11. 
Degree 1:

$$
C_{(\mathrm{f} 1)}=\frac{6 z^{2}}{z-1}, \quad C_{(\mathrm{f} 2)}=-\frac{6}{z(z-1)}
$$

Degree 2:

$$
\begin{aligned}
& C_{(\mathrm{f} 3)}=\frac{18 z(z+2)(2 z+1)}{(z-1)(2 z-1)}, \quad C_{(\mathrm{f} 4)}=\frac{18(z+2)(2 z+1)}{z(z-1)(z-2)}, \\
& C_{(\mathrm{f} 5)}=C_{(\mathrm{f} 6)}=0, \quad C_{(\mathrm{f} 7)}=-\frac{45 z^{3}}{2(z-1)(z-2)}, \\
& C_{(\mathrm{f} 8)}=-\frac{45}{2 z(z-1)(2 z-1)}, \quad C_{(\mathrm{f} 9)}=\frac{3 z^{2}}{z-1}, \\
& C_{(\mathrm{f} 10)}=-\frac{3}{z(z-1)} .
\end{aligned}
$$

Degree 3:

$$
C_{(\mathrm{f} 11)}=\frac{27 z(z+2)^{2}(2 z+1)^{2}}{(z-1)^{3}(2 z-1)}, \quad C_{(\mathrm{f} 12)}=\frac{27(z+2)^{2}(2 z+1)^{2}}{z(z-1)^{3}(z-2)}
$$

-

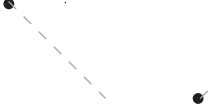

degree 1

(f1)

(f2)
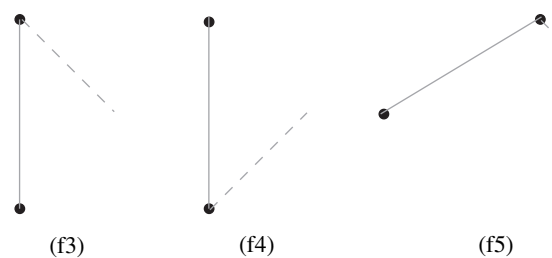

(f5)

(f6)

degree 2
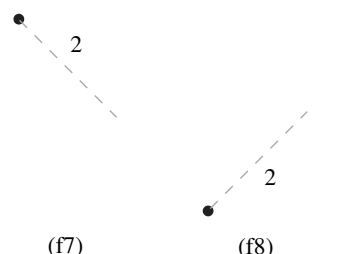

(f8)

(f9)

(f10)

Figure 9: Stable maps: degree 1 and 2 fiber class. 


$$
\begin{aligned}
& C_{(\mathrm{f} 13)}=C_{(\mathrm{f} 14)}=0, \quad C_{(\mathrm{f} 15)}=\frac{27(z+2)^{2}(2 z+1)^{2}}{(z-1)^{3}}, \\
& C_{(\mathrm{f} 16)}=-\frac{27(z+2)^{2}(2 z+1)^{2}}{z(z-1)^{3}}, \quad C_{(\mathrm{f} 17)}=C_{(\mathrm{f} 18)}=0, \\
& C_{(\mathrm{f} 19)}=C_{(\mathrm{f} 20)}=0, \quad C_{(\mathrm{f} 21)}=-\frac{27 z(z+2)(z+5)(2 z+1)(5 z+1)}{(z-1)^{3}(3 z-1)}, \\
& C_{(\mathrm{f} 22)}=-\frac{27(z+2)(z+5)(2 z+1)(5 z+1)}{z(z-1)^{3}(z-3)}, \quad C_{(\mathrm{f} 23)}=C_{(\mathrm{f} 24)}=C_{(\mathrm{f} 25)}=0 \\
& C_{(\mathrm{f} 26)}=C_{(\mathrm{f} 27)}=C_{(\mathrm{f} 28)}=0, \quad C_{(\mathrm{f} 29)}=\frac{18 z(z+2)(2 z+1)}{(z-1)^{2}}, \\
& C_{(\mathrm{f} 30)}=\frac{18(z+2)(2 z+1)}{z(z-1)^{2}}, \quad C_{(\mathrm{f} 31)}=C_{(\mathrm{f} 32)}=0, \\
& C_{(\mathrm{f} 33)}=\frac{36 z(z+2)(2 z+1)}{(z-1)^{2}(z-2)(2 z-1)}, \quad C_{(\mathrm{f} 34)}=-\frac{270 z^{2}(z+2)(2 z+1)}{(z-1)(z-2)(3 z-2)}, \\
& C_{(\mathrm{f} 35)}=\frac{270(z+2)(2 z+1)}{z(z-1)(2 z-1)(2 z-3)}, \quad C_{(\mathrm{f} 36)}=\frac{4 z^{2}}{z-1}, \\
& C_{(\mathrm{f} 37)}=-\frac{4}{z(z-1)}, \quad C_{(\mathrm{f} 38)}=-\frac{60 z^{3}}{(z-1)(z-2)}, \\
& C_{(\mathrm{f} 39)}=-\frac{60}{z(z-1)(2 z-1)}, \quad C_{(\mathrm{f} 40)}=\frac{1120 z^{4}}{3(z-1)(z-3)(2 z-3)}, \\
& C_{(\mathrm{f} 41)}=-\frac{1120}{3 z(z-1)(3 z-1)(3 z-2)} .
\end{aligned}
$$

We have identical corrections for vertical maps to the fiber $F_{2}$, except that the holonomy variables are different. Collecting all localization results, we can write the vertical instanton corrections in the form

$$
\begin{aligned}
F_{\text {inst }}^{(f)}= & -\mathrm{i} a_{\left(t_{1}^{\prime}\right)} q_{1}^{\prime}-\mathrm{i} a_{\left(t_{1}, t_{1}^{\prime}\right)} q_{1} q_{1}^{\prime} \\
& -\mathrm{i} a_{\left(2 t_{1}^{\prime}\right)} q_{1}^{\prime 2}-\mathrm{i} a_{\left(2 t_{1}, t_{1}^{\prime}\right)} q_{1}^{2} q_{1}^{\prime}-\mathrm{i} a_{\left(t_{1}, 2 t_{1}^{\prime}\right)} q_{1} q_{1}^{\prime 2}-\mathrm{i} a_{\left(3 t_{1}^{\prime}\right)} q_{1}^{\prime 3},
\end{aligned}
$$

where

$$
\begin{aligned}
a_{\left(t_{1}^{\prime}\right)}= & \frac{1}{g_{\mathrm{s}}}\left[C_{(\mathrm{f} 1)}\left(\operatorname{Tr} V_{1}^{\prime}+\operatorname{Tr} V_{2}^{\prime \prime}\right)+C_{(\mathrm{f} 2)}\left(\operatorname{Tr} V_{1}^{\prime \prime}+\operatorname{Tr} V_{2}^{\prime}\right)\right], \\
a_{\left(t_{1}, t_{1}^{\prime}\right)}= & \frac{1}{g_{\mathrm{s}}}\left[\left(C_{(\mathrm{f} 3)}+C_{(\mathrm{f} 5)}\right)\left(\operatorname{Tr} V_{1}^{\prime}+\operatorname{Tr} V_{2}^{\prime \prime}\right)+\left(C_{(\mathrm{f} 4)}+C_{(\mathrm{f} 6)}\right)\right. \\
& \left.\times\left(\operatorname{Tr} V_{1}^{\prime \prime}+\operatorname{Tr} V_{2}^{\prime}\right)\right],
\end{aligned}
$$



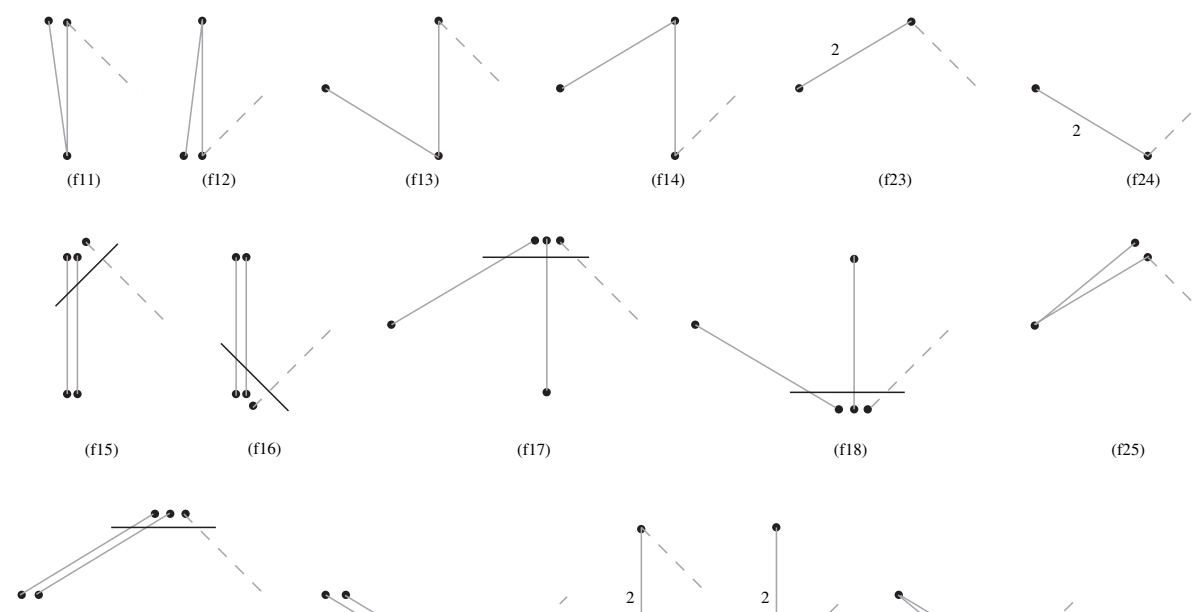

(f19)
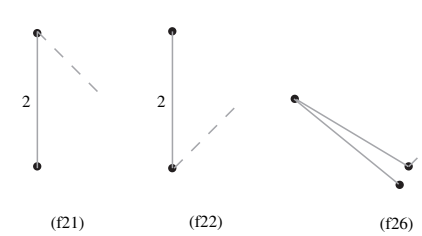

Figure 10: Stable maps: degree 3 fiber class-I.

$$
\begin{aligned}
a_{\left(2 t_{1}^{\prime}\right)}= & \frac{1}{g_{\mathrm{s}}}\left[C_{(\mathrm{f} 7)}\left(\operatorname{Tr} V_{1}^{\prime 2}+\operatorname{Tr} V_{2}^{\prime \prime 2}\right)+C_{(\mathrm{f} 8)}\left(\operatorname{Tr} V_{1}^{\prime \prime 2}+\operatorname{Tr} V_{2}^{\prime 2}\right)\right] \\
& -\mathrm{i} C_{(\mathrm{f} 9)}\left[\left(\operatorname{Tr} V_{1}^{\prime}\right)^{2}+\left(\operatorname{Tr} V_{2}^{\prime \prime}\right)^{2}\right]-\mathrm{i} C_{(\mathrm{f} 10)}\left[\left(\operatorname{Tr} V_{1}^{\prime \prime}\right)^{2}+\left(\operatorname{Tr} V_{2}^{\prime}\right)^{2}\right],
\end{aligned}
$$

$$
\begin{aligned}
& a_{\left(2 t_{1}, t_{1}^{\prime}\right)}=\frac{1}{g_{\mathrm{s}}}\left[\sum_{\substack{k=1 \\
k \text { odd }}}^{27} C_{(\mathrm{f} k)}\left(\operatorname{Tr} V_{1}^{\prime}+\operatorname{Tr} V_{2}^{\prime \prime}\right)+\sum_{\substack{k=2 \\
k \text { even }}}^{28} C_{(\mathrm{f} k)}\left(\operatorname{Tr} V_{1}^{\prime \prime}+\operatorname{Tr} V_{2}^{\prime}\right)\right], \\
& a_{\left(t_{1}, 2 t_{1}^{\prime}\right)}=-\mathrm{i}\left(C_{(\mathrm{f} 29)}+C_{(\mathrm{f} 31)}\right)\left[\left(\operatorname{Tr} V_{1}^{\prime}\right)^{2}+\left(\operatorname{Tr} V_{2}^{\prime \prime}\right)^{2}\right]
\end{aligned}
$$$$
-\mathrm{i}\left(C_{(\mathrm{f} 30)}+C_{(\mathrm{f} 32)}\right)\left[\left(\operatorname{Tr} V_{1}^{\prime \prime}\right)^{2}+\left(\operatorname{Tr} V_{2}^{\prime}\right)^{2}\right]
$$$$
-\mathrm{i} C_{(\mathrm{f} 33)}\left(\operatorname{Tr} V_{1}^{\prime} \operatorname{Tr} V_{1}^{\prime \prime}+\operatorname{Tr} V_{2}^{\prime \prime} \operatorname{Tr} V_{2}^{\prime}\right)
$$$$
+\frac{1}{g_{\mathrm{s}}}\left[C_{(\mathrm{f} 34)}\left(\operatorname{Tr} V_{1}^{\prime 2}+\operatorname{Tr} V_{2}^{\prime \prime 2}\right)\right.
$$$$
\left.+C_{(\mathrm{f} 35)}\left(\operatorname{Tr} V_{1}^{\prime \prime 2}+\operatorname{Tr} V_{2}^{\prime 2}\right)\right] \text {, }
$$

$$
\begin{aligned}
a_{\left(3 t_{1}^{\prime}\right)=} & -g_{\mathrm{s}}\left[C_{(\mathrm{f} 36)}\left[\left(\operatorname{Tr} V_{1}^{\prime}\right)^{3}+\left(\operatorname{Tr} V_{2}^{\prime \prime}\right)^{3}\right]+C_{(\mathrm{f} 37)}\left[\left(\operatorname{Tr} V_{1}^{\prime \prime}\right)^{3}+\left(\operatorname{Tr} V_{2}^{\prime}\right)^{3}\right]\right] \\
& -\mathrm{i} C_{(\mathrm{f} 38)}\left(\operatorname{Tr} V_{1}^{\prime} \operatorname{Tr} V_{1}^{\prime 2}+\operatorname{Tr} V_{2}^{\prime \prime} \operatorname{Tr} V_{2}^{\prime \prime 2}\right)
\end{aligned}
$$$$
-\mathrm{i} C_{(\mathrm{f} 39)}\left(\operatorname{Tr} V_{1}^{\prime \prime} \operatorname{Tr} V_{1}^{\prime \prime 2}+\operatorname{Tr} V_{2}^{\prime} \operatorname{Tr} V_{2}^{\prime 2}\right)
$$$$
+\frac{1}{g_{\mathrm{s}}}\left[C_{(\mathrm{f} 40)}\left(\operatorname{Tr} V_{1}^{\prime 3}+\operatorname{Tr} V_{2}^{\prime \prime 3}\right)+C_{(\mathrm{f} 41)}\left(\operatorname{Tr} V_{1}^{\prime \prime 3}+\operatorname{Tr} V_{2}^{\prime 3}\right)\right] \text {. }
$$ 


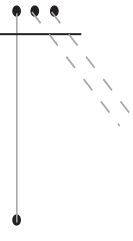

(f29)

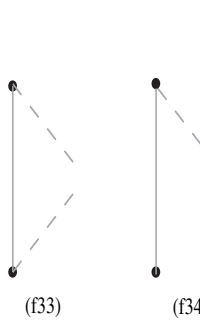

(f33)

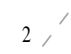

6

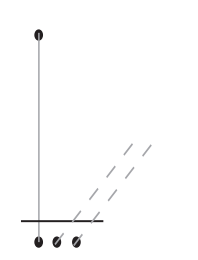

(f30)

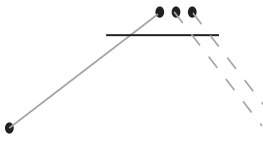

31)

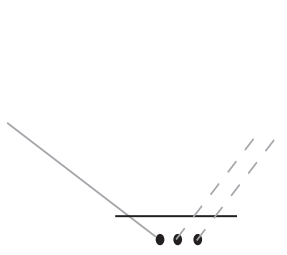

(f32)

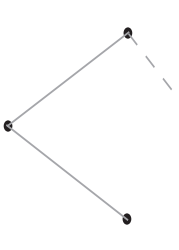

(f27)
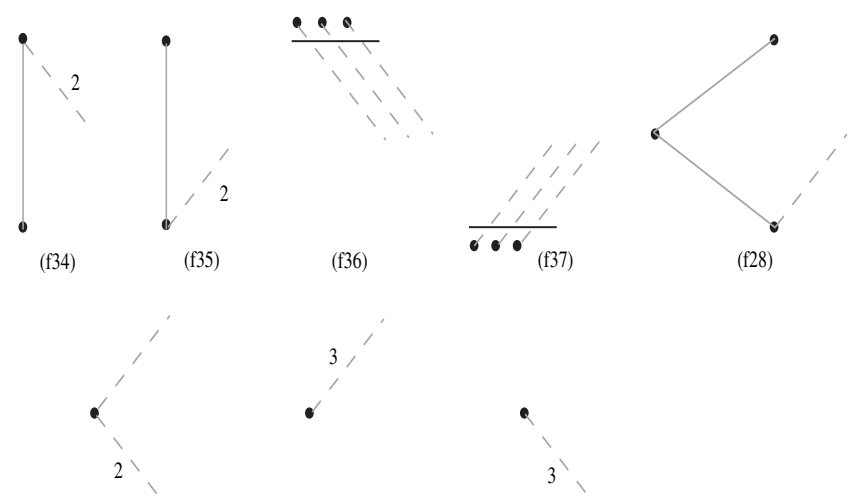

(f39)
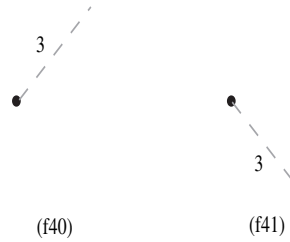

(f41)

Figure 11: Stable maps: degree 3 fiber class-II.

Some points in this formula deserve explanation. As we mentioned earlier, for each disc $\Delta_{a}$ of the domain we have to write down an instanton factor of the form $\mathrm{e}^{-d_{a} \tau_{\rho(a)}}$, where $\tau_{\rho(a)}$ are flat open string Kähler parameters, which are generally different from the flat closed string moduli. For the vertical classes under consideration, we should have four such parameters $\tau_{1}^{\prime}, \tau_{1}^{\prime \prime}, \tau_{2}^{\prime}$ and $\tau_{2}^{\prime \prime}$ corresponding to $D_{1}^{\prime}, D_{1}^{\prime \prime}, D_{2}^{\prime}$ and $D_{2}^{\prime \prime}$. These parameters depend linearly on the framing of the corresponding knots, hence a priori they have different values although all vertical discs are in the same homology class. However, once we absorb the trivial framing dependence by redefining the holonomy variables, as explained below equation (5.23), we can take them to be equal. We let $q_{1}^{\prime}=\mathrm{e}^{-\tau_{1}^{\prime}}=\mathrm{e}^{-\tau_{1}^{\prime \prime}}=\mathrm{e}^{-\tau_{2}^{\prime}}=\mathrm{e}^{-\tau_{2}^{\prime \prime}}$ and $q_{1}=\mathrm{e}^{-t_{1}}$ be the closed string instanton factor for the vertical class $h_{1}$.

The next step is to compute the Chern-Simons free energy including the instanton corrections (7.17). All link and knot invariants should be expanded in powers of $g_{\mathrm{s}}$ as explained in Section 5. The resulting expansion will automatically be a power series of the framings $p_{1}^{\prime}, p_{1}^{\prime \prime}, p_{2}^{\prime}$ and $p_{2}^{\prime \prime}$, which take the values (7.12). Let us first record the final result including corrections 
up to second order in $q_{1}$ and $q_{1}^{\prime}$

$$
\begin{aligned}
g_{\mathrm{s}}^{2} \ln \left\langle\mathrm{e}^{F_{\text {inst }}^{(f 2)}}\right\rangle= & -12 q_{1}^{\prime}\left(y-y^{-1}\right) \frac{z^{2}+z+1}{z} \\
& +\frac{3}{2}\left(q_{1}^{\prime}\right)^{2} y^{2} \frac{14 z^{4}+51 z^{3}+173 z^{2}+51 z+14}{z(z-2)(2 z-1)} \\
& +3\left(q_{1}^{\prime}\right)^{2} \frac{-8 z^{4}-24 z^{3}-86 z^{2}-24 z-8}{z(z-2)(2 z-1)} \\
& +\frac{3}{2}\left(q_{1}^{\prime}\right)^{2} y^{-2} \frac{2 z^{4}-3 z^{3}-z^{2}-3 z+2}{z(z-2)(2 z-1)} \\
& +3 q_{1}^{\prime} q_{1}\left(y-y^{-1}\right) \frac{-24 z^{4}-36 z^{3}+12 z^{2}-36 z-24}{z(z-2)(2 z-1)},
\end{aligned}
$$

where $y=\mathrm{e}^{\mathrm{i} \lambda / 2}$ denotes the exponentiated 't Hooft coupling constant of the two Chern-Simons theories supported on $\bar{L}_{1}$ and $\bar{L}_{2}$. (The two coupling constants must be equal as a result of the zero charge condition, as explained above equation (6.5).)

Having reached this point, we have to face a new puzzle: unlike the local examples studied in Section 5, the final answer is a function of $z$. If this answer has to be taken at face value, what is the correct interpretation? To shed some light on this question, let us perform a change of variables of the form

$$
q_{1}=\tilde{q}_{1} \tilde{q}_{4}, \quad y=\left(\tilde{q}_{1}\right)^{1 / 2}, \quad q_{1}^{\prime}=\left(\tilde{q}_{1}\right)^{1 / 2} \tilde{q}_{4} .
$$

Note that the first two relations follow from the duality map (7.6). The third relation involving the open string instanton factor $q_{1}^{\prime}$ takes into account an extra shift in the Kähler parameters due to open string quantum corrections [15]. As a function of the new parameters, equation (7.19) becomes

$$
\begin{aligned}
g_{\mathrm{s}}^{2} \ln \left\langle\mathrm{e}^{F_{\text {inst }}^{(f 2)}}\right\rangle= & 12 \tilde{q}_{4}\left(1-\tilde{q}_{1}\right) \frac{z^{2}+z+1}{z}-\frac{3}{2} \tilde{q}_{1}^{2} \tilde{q}_{4}^{2} \frac{17 z^{2}+53 z+17}{z} \\
& +6 \tilde{q}_{1} \tilde{q}_{4}^{2} \frac{4 z^{2}+13 z+4}{z}+\frac{3}{2} \tilde{q}_{4}^{2} \frac{2 z^{4}-3 z^{3}-z^{2}-3 z+2}{z(z-2)(2 z-1)} .
\end{aligned}
$$

In order to understand the meaning of this expression in the context of large $N$ duality, it is very helpful to take a closer look at the closed string expansions on both sides, i.e., the series $\mathcal{F}_{Y ; \mathrm{cl}}^{(0)}\left(g_{\mathrm{s}}, t_{\alpha}\right)$ and $\mathcal{F}_{\widetilde{Y} ; \mathrm{cl}}^{(0)}\left(g_{\mathrm{s}}, \widetilde{t}_{\gamma}\right)$.

In both cases, the coefficients $C_{\beta}$ and $\widetilde{C}_{\widetilde{\beta}}$ can be computed by localization using the convex obstruction bundle approach reviewed in Section 3. We have studied in detail the $G$ action on $\mathcal{Z}$. There is a similar $\widetilde{G}=\left(S^{1}\right)^{8}$ action 
on $\widetilde{\mathcal{Z}}$ given by

$$
\left(\mathrm{e}^{\mathrm{i} \widetilde{\phi}_{1}}, \mathrm{e}^{\mathrm{i} \widetilde{\phi}_{2}}, \ldots, \mathrm{e}^{\mathrm{i} \widetilde{\phi}_{8}}\right) \cdot\left(\widetilde{Z}_{1}, \widetilde{Z}_{2}, \ldots, \widetilde{T}\right)=\left(\mathrm{e}^{\mathrm{i} \widetilde{\phi}_{1}} \widetilde{Z}_{1}, \mathrm{e}^{\mathrm{i} \widetilde{\phi}_{2}} \widetilde{Z}_{2}, \ldots, \mathrm{e}^{\mathrm{i} \widetilde{\phi}_{8}} \widetilde{T}\right)
$$

whose fixed locus consists of all isolated points on $\widetilde{\mathcal{Z}}$ which can be represented as a quadruple intersection of toric divisors. The $\widetilde{G}$-invariant curves on $\widetilde{\mathcal{Z}}$ form a skeleton which can be obtained from the skeleton of $\mathcal{Z}$ by replacing the fibers $F_{1}, \ldots, F_{4}$ with $\mathbb{F}_{1}$ surfaces $\widetilde{F}_{1}, \ldots, \widetilde{F}_{4}$. For later use, note that there is a lift of the action of $T$ to $\widetilde{\mathcal{Z}}$ given by

$$
\left(\mathrm{e}^{\mathrm{i} \tilde{\phi}_{1}}, \mathrm{e}^{\mathrm{i} \widetilde{\phi}_{2}}, \ldots, \mathrm{e}^{\mathrm{i} \widetilde{\phi}_{8}}\right)=\left(\mathrm{e}^{\mathrm{i} \lambda_{1} \phi}, 1, \mathrm{e}^{\mathrm{i} \lambda_{3} \phi}, 1, \mathrm{e}^{-\mathrm{i} \lambda_{1} \phi}, \mathrm{e}^{-\mathrm{i} \lambda_{3} \phi}, 1,1\right) .
$$

Now let us write down the vertical Gromov-Witten expansions for $Y$ and $\widetilde{Y}$. We have $\beta=n h_{1}, n \geq 0$, on $Y$ and $\widetilde{\beta}=n_{1} \widetilde{h}_{1}+n_{4} \widetilde{h}_{4}, n_{1}, n_{4} \geq 0$ on $\widetilde{Y}$. Therefore, we obtain

$$
\mathcal{F}_{Y ; \mathrm{cl}}^{(0), f}\left(g_{s}, t_{1}\right)=\sum_{n \geq 0} C_{n} q_{1}^{n}, \quad \mathcal{F}_{\widetilde{Y} ; \mathrm{cl}}^{(0), f}=\sum_{n_{1}, n_{4} \geq 0} \widetilde{C}_{n_{1} n_{4}} \tilde{q}_{1}^{n_{1}} \tilde{q}_{4}^{n_{4}}
$$

where the coefficients $C_{n}$ and $\widetilde{C}_{n_{1} n_{4}}$ have integral representations of the form

$$
C_{n}=\int_{\left[\bar{M}_{0,0}(\mathcal{Z}, \beta)\right]} e(\mathcal{V}), \quad \widetilde{C}_{n_{1} n_{4}}=\int_{\left[\bar{M}_{0,0}(\widetilde{\mathcal{Z}}, \widetilde{\beta})\right]} e(\widetilde{\mathcal{V}}),
$$

where $\mathcal{V}$ and $\widetilde{\mathcal{V}}$ are the obstruction bundles.

Given the structure of the toric skeleton in both cases the fixed loci in the moduli spaces $\bar{M}_{0,0}(\mathcal{Z}, \beta)$ and $\bar{M}_{0,0}(\widetilde{\mathcal{Z}}, \widetilde{\beta})$ fall naturally in four classes, depending on their image in $\mathcal{Z}, \widetilde{\mathcal{Z}}$. A component of the fixed locus in $\bar{M}_{0,0}(\mathcal{Z}, \beta)$ will be called of type $i, i=1, \ldots, 4$, if its image is embedded in the invariant fiber $F_{i} \subset \mathcal{Z}$. Similarly, a component of the fixed locus in $\bar{M}_{0,0}(\widetilde{\mathcal{Z}}, \widetilde{\beta})$ will be called of type $i, i=1, \ldots, 4$, if its image is embedded in the invariant fiber $\widetilde{F}_{i} \subset \widetilde{\mathcal{Z}}$. The coefficients $C_{n}$ and $\widetilde{C}_{n_{1} n_{4}}$ receive contributions from all such fixed loci, i.e., we have

$$
\begin{aligned}
C_{n} & =i_{p t}^{*} \sum_{i=1}^{4} \sum_{\Xi_{i}} \int_{\left[\Xi_{i}\right]_{G}} \frac{e_{G}\left(\mathcal{V}_{\Xi_{i}}\right)}{e_{G}\left(N_{\Xi_{i}}\right)}, \\
\widetilde{C}_{n_{1} n_{4}} & =i_{p t}^{*} \sum_{i=1}^{4} \sum_{\widetilde{\Xi}_{i}} \int_{\left[\widetilde{\Xi}_{i}\right]_{\widetilde{G}}} \frac{e_{\widetilde{G}}\left(\widetilde{\mathcal{V}}_{\widetilde{\Xi}_{i}}\right)}{e_{\widetilde{G}}\left(N_{\widetilde{\Xi}_{i}}\right)} .
\end{aligned}
$$

The local contributions in equation (7.26) are homogeneous rational functions of degree zero of the torus weights. In order to understand the meaning of the open string expansion (7.21), we will specialize these local contributions to $\mathcal{K}_{T}$ as explained below equation (6.49), obtaining rational functions of $z$. 
Naively, large $N$ duality predicts a relation of the form

$$
\sum_{n_{1}, n_{4} \geq 0} \widetilde{C}_{n_{1} n_{4}} \tilde{q}_{1}^{n_{1}} \tilde{q}_{4}^{n_{4}}=\sum_{n \geq 0} C_{n} q_{1}^{n}+\mathcal{F}_{\mathrm{CS}, 1}^{(0)}+\mathcal{F}_{\mathrm{CS}, 2}^{(0)}+\ln \left\langle\mathrm{e}^{F_{\text {inst }}^{(\mathrm{f} 2)}}\right\rangle
$$

where the coefficients $C_{n}$ and $\widetilde{C}_{n_{1} n_{4}}$ are given by equation (7.26). Clearly, such a relation cannot be true since $C_{n}$ and $\widetilde{C}_{n_{1} n_{4}}$ are rational numbers, while the coefficients in the open string expansion are functions of $z$. However, let us recall an interesting geometric fact. The closed component $\Sigma_{0}$ of the open string maps which contribute to equation (7.21) is mapped either to $F_{1}$ or to $F_{2}$. There are no such maps to $F_{3}$ and $F_{4}$ since all these fibers are supported away from the vertical discs. This suggests that in (7.27) one should take a similar truncation of the coefficients $C_{n}$ and $\widetilde{C}_{n_{1} n_{4}}$ by only summing over invariant maps to $F_{1}, F_{2}$ and, respectively, $\widetilde{F}_{1}, \widetilde{F}_{2}$. More precisely, the truncated coefficients are given by

$$
\begin{array}{r}
C_{n}^{\mathrm{tr}}=i_{p t}^{*} s^{*} \sum_{i=1}^{2} \sum_{\Xi_{i}} \int_{\left[\Xi_{i}\right]_{G}} \frac{e_{G}\left(\mathcal{V}_{\Xi_{i}}\right)}{e_{G}\left(N_{\Xi_{i}}\right)}, \\
\widetilde{C}_{n_{1} n_{4}}^{\mathrm{tr}}=i_{p t}^{*} \widetilde{s}^{*} \sum_{i=1}^{2} \sum_{\widetilde{\Xi}_{i}} \int_{\left[\widetilde{\Xi}_{i}\right]_{\widetilde{G}}} \frac{e_{\widetilde{G}}\left(\widetilde{\mathcal{V}}_{\widetilde{\Xi}_{i}}\right)}{e_{\widetilde{G}}\left(N_{\widetilde{\Xi}_{i}}\right)},
\end{array}
$$

where $s^{*}: \mathcal{K}_{G} \longrightarrow \mathcal{K}_{T}$ and $\widetilde{s}^{*}: \mathcal{K}_{\widetilde{G}} \longrightarrow \mathcal{K}_{T}$ are specialization morphisms. Clearly these are no longer rational numbers since we do not sum over all fixed loci. After specialization to $\mathcal{K}_{T}$ we will obtain rational functions of $z$, as discussed before. One can regard $C_{n}^{\mathrm{tr}}$ and $\widetilde{C}_{n_{1} n_{4}}^{\mathrm{tr}}$ as equivariant refinements of standard Gromov-Witten invariants which probe a finer structure of the moduli space of maps. Then, we propose the following modified large $N$ duality conjecture

$$
\sum_{n_{1}, n_{4} \geq 0} \widetilde{C}_{n_{1} n_{4}}^{\mathrm{tr}} \tilde{q}_{1}^{n_{1}} \tilde{q}_{4}^{n_{4}}=\sum_{n \geq 0} C_{n}^{\mathrm{tr}} q_{1}^{n}+\mathcal{F}_{\mathrm{CS}, 1}^{(0)}+\mathcal{F}_{\mathrm{CS}, 2}^{(0)}+\ln \left\langle e^{F_{\text {inst }}^{(f 2)}}\right\rangle .
$$

Ideally, one would like to have a conceptual proof of this conjecture, which seems to be very difficult. Here we will restrict ourselves to a numerical test. The truncated closed string expansions can be evaluated by summing over Kontsevich graphs [36]. This is a standard computation, so we will omit the 
details. Up to second degree terms, one finds the following expressions

$$
\begin{aligned}
\sum_{n \geq 0} C_{n}^{\operatorname{tr}} q_{1}^{n}= & 18 \frac{(z+2)(2 z+1)}{z} q_{1}+\frac{81}{4} \frac{2 z^{2}+5 z+2}{z} q_{1}^{2}+\mathcal{O}\left(q_{1}^{3}\right), \\
\sum_{n_{1}, n_{4} \geq 0} \widetilde{C}_{n_{1} n_{4}}^{\operatorname{tr}} \tilde{q}_{1}^{n_{1}} \tilde{q}_{4}^{n_{4}}= & 2 \tilde{q}_{1}+\frac{1}{4} \tilde{q}_{1}^{2}+12 \frac{z^{2}+z+1}{z} \tilde{q}_{4} \\
& +\frac{3}{2} \frac{2 z^{4}-3 z^{3}-z^{2}-3 z+2}{z(z-2)(2 z-1)} \tilde{q}_{4}^{2} \\
& +6 \frac{4 z^{2}+13 z+4}{z} \tilde{q}_{1} \tilde{q}_{4}+\frac{3}{4} \frac{20 z^{2}+29 z+20}{z} \tilde{q}_{1}^{2} \tilde{q}_{4}^{2} \\
& +\mathcal{O}\left(\tilde{q}_{1}^{3}, \ldots, \tilde{q}_{4}^{3}\right) .
\end{aligned}
$$

Using the duality relation $q_{1}=\tilde{q}_{1} \tilde{q}_{4}$ and equation (7.21), it is straightforward to check that equation (7.29) is satisfied up to terms of degree 2. This is positive evidence for the modified duality conjecture. Obviously, one would like to test this conjecture at higher order in the expansion. The main problem is that the closed string computations become very tedious since we have to sum over large numbers of graphs. Although a more thorough investigation is possible, it would be preferable to develop a more conceptual approach. We leave this aspect for future work.

Before we can continue our analysis, we should try to understand the meaning of the modified duality conjecture. The truncation (7.30) seems to be necessary because the open string expansion cannot capture all the closed string information. For pure geometric reasons, on the open string side, we cannot take into account the effect of the fixed loci mapping to $\widetilde{F}_{3}$ and $\widetilde{F}_{4}$. Clearly, this phenomenon is very specific to the present example. In other examples, one may find that various truncations of Gromov-Witten invariants are needed. For example, we expect that no truncation is necessary for the compact $d P_{5}$ model described in Section 4. This is so because in that case we have four vanishing cycles rather than two, and the invariant open string maps can take values in the fibers $F_{3}$ and $F_{4}$ as well. The general assertion one could make in this context is that the open string expansion computes the sum over those closed string graphs which are geometrically accessible. The notion of geometrically accessible graphs depends on the peculiarities of the model. We expect that such a notion and a refined duality conjecture can be formulated for all transitions in which the nodal points are fixed points of the generic torus action. This is a very interesting subject for future work.

Returning to our model, there is an important observation one could make. While the unrefined duality conjecture (7.27) cannot be true for 
arbitrary values of $z$, quite remarkably, it holds true for the special value $z=1$ ! First note that if we specialize $z=1$ in equation (7.30) we obtain

$$
\begin{aligned}
\sum_{n \geq 0} C_{n}^{\mathrm{tr}} q_{1}^{n} & =162 q_{1}+\frac{729}{4} q_{1}^{2}+\cdots, \sum_{n_{1}, n_{4} \geq 0} \widetilde{C}_{n_{1} n_{4}}^{\mathrm{tr}} \tilde{q}_{1}^{n_{1}} \tilde{q}_{4}^{n_{4}} \\
& =2 \tilde{q}_{1}+\frac{1}{4} \tilde{q}_{1}^{2}+36 \tilde{q}_{4}+\frac{9}{2} \tilde{q}_{4}^{2}+126 \tilde{q}_{1} \tilde{q}_{4}+\frac{207}{4} \tilde{q}_{1}^{2} \tilde{q}_{4}^{2}+\cdots
\end{aligned}
$$

Although written in terms of truncated coefficients, these are the full genus zero Gromov-Witten expansions of the two models (see Appendix A). The explanation is that for $z=1$, the contributions of the fixed loci of types 3 and 4 cancel each other, leaving only the contributions of fixed loci of types 1 and 2. This phenomenon is not very uncommon in Gromov-Witten theory. Sometimes one can exploit the symmetry properties of the target space to simplify the local contributions of the fixed loci by making a special choice of weights (see, e.g., [19]).

Since the refined duality conjecture was shown to be true up to order 2 for any $z$, it follows that the unrefined conjecture also holds up to order 2 if we set $z=1$. Exploiting this feature, we can perform higher-order tests of the duality more efficiently. Below we record the open string expansion including terms up to order 3 in Kähler classes for $z=1$

$$
\begin{aligned}
g_{\mathrm{s}}^{2} \ln \left\langle e^{F_{\text {inst }}^{(f)}}\right\rangle= & -36 q_{1}^{\prime}\left(y-y^{-1}\right)+324 q_{1} q_{1}^{\prime}\left(y-y^{-1}\right) \\
& +450 q_{1}^{\prime 2}+\frac{9}{2} q_{1}^{\prime 2} y^{-2}-\frac{909}{2} q_{1}^{\prime 2} y^{2} \\
& +8748 q_{1}^{2} q_{1}^{\prime}\left(y-y^{-1}\right)-6804 q_{1} q_{1}^{\prime 2}+5346 q_{1} q_{1}^{\prime 2} y^{-2} \\
& +8262 q_{1} q_{1}^{\prime 2} y^{2}+\frac{4}{3} q_{1}^{\prime 3} y^{-3}-5344 q_{1}^{\prime 3} y^{-1} \\
& +22508 q_{1}^{\prime 3} y-\frac{51496}{3} q_{1}^{\prime 3} y^{3}+\cdots
\end{aligned}
$$

Using the duality map (7.20), we can rewrite this expression in the following form

$$
\begin{aligned}
g_{\mathrm{s}}^{2} \ln \left\langle e^{\left.F_{\mathrm{inst}}^{(f)}\right\rangle=}\right. & 36 \tilde{q}_{4}-36 \tilde{q}_{1} \tilde{q}_{4}+\frac{9}{2} \tilde{q}_{4}^{2}+126 \tilde{q}_{1} \tilde{q}_{4}^{2}-\frac{261}{2} \tilde{q}_{1}^{2} \tilde{q}_{4}^{2} \\
& +\frac{4}{3} \tilde{q}_{4}^{3}+2 \tilde{q}_{1} \tilde{q}_{4}^{3}+152 \tilde{q}_{1}^{2} \tilde{q}_{4}^{3}-\frac{466}{3} \tilde{q}_{1}^{3} \tilde{q}_{4}^{3}+\cdots
\end{aligned}
$$




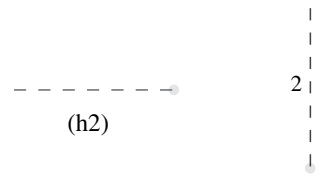

(h3) (h1)

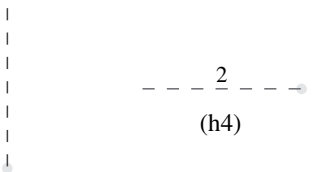

(h4)

3)

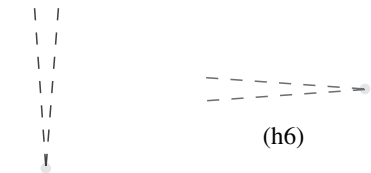

(h5)
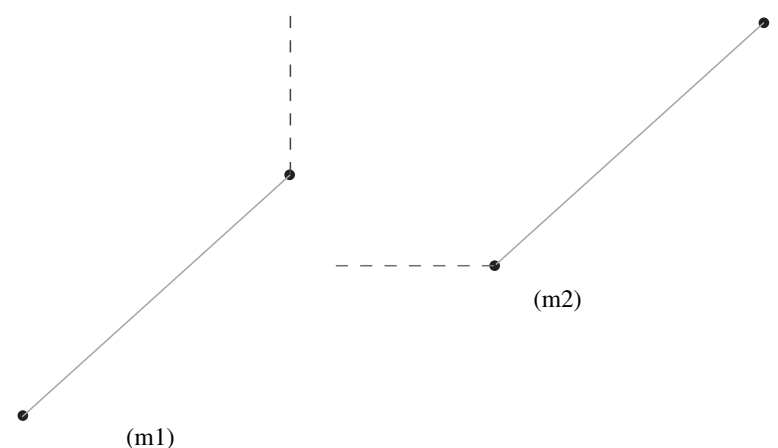

Figure 12: Stable maps: degree 2 mixed and degree 1 and 2 horizontal classes. The color coding is as before.

This formula is to be compared to the closed string genus zero expansion worked out in Appendix A. Before running this test, it is more convenient to compute the open string expansions for mixed and horizontal classes as well.

\subsection{Mixed and horizontal classes}

We have to perform analogous computations for instanton corrections in homology classes of the form $\beta=n_{1} h_{1}+n_{2} h_{2}+n_{3} h_{3}$ with $n_{1}, n_{2}, n_{3} \geq 0$. The technology is very similar, except that now one has to consider horizontal discs as well. The equivariant integrals (7.9) can be computed as above using the graph method. We record below the instanton expansion and enumerate the relevant graphs, which should be interpreted as explained below equations (6.48) and (7.13).

The closed string data are encoded in continuous line segments representing irreducible components of the domain. Each such component is either contracted or mapped to an invariant curve in $\mathcal{Z}$ as indicated by color, inclination angle and degree. The conventions for the degree of the map are the same as before, i.e., for $d=0,1$ the segment is left unmarked, while for $d \geq 2$ the segment is marked. The extra legs drawn with dashed colored lines represent the open string contributions to the instanton coefficients 
(equation (7.9)). The colors correspond to different homology classes, as explained below figure 6 . For example, the graphs in the first line of figure 12 represent pure horizontal open string contributions. The vertical blue lines correspond to multicover contributions of either $D_{3}^{\prime}$ or $D_{3}^{\prime \prime}$, and the horizontal red lines correspond to multicover contributions of $D_{4}^{\prime}$ or $D_{4}^{\prime \prime}$. Due to the symmetry properties of $\mathcal{Z}$, the moduli space integrals are identical for $D_{3}^{\prime}$ and $D_{3}^{\prime \prime}$ and respectively, $D_{4}^{\prime}$ and $D_{4}^{\prime \prime}$. However, the holonomy variables are different. This is reflected in the coefficients $a_{\left(t_{2}^{\prime}\right)}$ and $a_{\left(t_{3}^{\prime}\right)}$ in equation (7.34). (h7) is again a pure open string contribution representing a $1: 1$ cover of either $D_{3}^{\prime} \cup_{Q_{3}} D_{3}^{\prime \prime}$ or $D_{4}^{\prime} \cup_{Q_{4}} D_{4}^{\prime \prime}$. This gives rise to $a_{\left(t_{2}^{\prime}, t_{3}^{\prime}\right)}$. (m1) represents a contribution of the form (7.9) with $h=1, a=1, \rho(1)=3^{\prime}$ (or $\left.\rho(1)=4^{\prime \prime}\right), d_{3}^{\prime}=1$ (or $d_{4}^{\prime}=1$ ) and $\beta=h_{1}+h_{2}$. Therefore, we have one degree 1 disc factor corresponding to a 1:1 cover of either $D_{3}^{\prime}$ or $D_{4}^{\prime}$ and an equivariant integral on $\bar{M}_{0,1}\left(\mathcal{Z}, h_{1}\right)$ which localizes on a vertical curve in $F_{3}$ $\left(F_{4}\right)$ passing through $P_{3}^{\prime}\left(P_{4}^{\prime \prime}\right)$. There are two such curves $\overline{P_{3}^{\prime} P_{3}}$ and $\overline{P_{3}^{\prime} P_{3}^{\prime \prime}}$ in $F_{3}$, respectively, $\overline{P_{4}^{\prime \prime} P_{4}}$ and $\overline{P_{4}^{\prime \prime} P_{4}^{\prime}}$ in $F_{4}$. However, it can be easily checked that whenever a component maps to either $\overline{P_{3}^{\prime} P_{3}}$ or $\overline{P_{4}^{\prime \prime} P_{4}}$, the contribution of the corresponding graph vanishes identically upon specialization to $\mathcal{K}_{T}$. This follows form the fact that these curves are fixed under $T$, hence the tangent toric weight vanishes. Such graphs will not be included in the figures. Overall, we are left with the contribution of the vertical curves $\overline{P_{3}^{\prime} P_{3}^{\prime \prime}}$ and $\overline{P_{4}^{\prime \prime} P_{4}^{\prime}}$; they determine the term $a_{\left(t_{1}, t_{2}^{\prime}\right)}$. The last graph $(\mathrm{m} 2)$ is very similar and yields $a_{\left(t_{1}, t_{3}^{\prime}\right)}$. The same rules apply to the third degree graphs represented in figures 13 and 14. The final expression for all relevant instanton corrections is ${ }^{11}$

$$
\begin{aligned}
F_{\text {inst }}^{(m, h)}= & -\mathrm{i} a_{\left(t_{1}^{\prime}\right)} q_{1}^{\prime}-\mathrm{i} a_{\left(t_{2}^{\prime}\right)} q_{2}^{\prime}-\mathrm{i} a_{\left(t_{3}^{\prime}\right)} q_{3}^{\prime}-\mathrm{i} a_{\left(t_{1}, t_{1}^{\prime}\right)} q_{1} q_{1}^{\prime}-\mathrm{i} a_{\left(t_{1}, t_{2}^{\prime}\right)} q_{1} q_{2}^{\prime} \\
& -\mathrm{i} a_{\left(t_{1}, t_{3}^{\prime}\right)} q_{1} q_{3}^{\prime}-\mathrm{i} a_{\left(2 t_{1}^{\prime}\right)} q_{1}^{\prime 2}-\mathrm{i} a_{\left(2 t_{2}^{\prime}\right)} q_{2}^{\prime 2}-\mathrm{i} a_{\left(2 t_{3}^{\prime}\right)} q_{3}^{\prime 2}-\mathrm{i} a_{\left(t_{2}, t_{3}^{\prime}\right)} q_{2}^{\prime} q_{3}^{\prime} \\
& -\mathrm{i} a_{\left(2 t_{1}, t_{2}^{\prime}\right)} q_{1}^{2} q_{2}^{\prime}-\mathrm{i} a_{\left(2 t_{1}, t_{3}^{\prime}\right)} q_{1}^{2} q_{3}^{\prime}-\mathrm{i} a_{\left(t_{1}, t_{2}, t_{1}^{\prime}\right)} q_{1} q_{2} q_{1}^{\prime} \\
& -\mathrm{i} a_{\left(t_{1}, t_{2}, t_{2}^{\prime}\right)} q_{1} q_{2} q_{2}^{\prime}-\mathrm{i} a_{\left(t_{1}, t_{2}, t_{3}^{\prime}\right)} q_{1} q_{2} q_{3}^{\prime}-\mathrm{i} a_{\left(t_{1}, t_{3}, t_{1}^{\prime}\right)} q_{1} q_{3} q_{1}^{\prime} \\
& -\mathrm{i} a_{\left(t_{1}, t_{3}, t_{2}^{\prime}\right)} q_{1} q_{3} q_{2}^{\prime}-\mathrm{i} a_{\left(t_{1}, t_{3}, t_{3}^{\prime}\right)} q_{1} q_{3} q_{3}^{\prime} \\
& -\mathrm{i} a_{\left(t_{1}, 2 t_{2}\right)} q_{1} q_{2}^{\prime 2}-\mathrm{i} a_{\left(t_{1}, 2 t_{3}^{\prime}\right)} q_{1} q_{3}^{\prime 2}
\end{aligned}
$$

\footnotetext{
${ }^{11}$ Note that in this formula we have to include pure vertical corrections up to degree 2 as well. Such terms multiply the existing mixed and horizontal corrections in the ChernSimons expansion giving rise to new mixed terms in the final answer.
} 
where

$$
\begin{aligned}
& a_{\left(t_{2}^{\prime}\right)}=-\frac{1}{g_{s}} C_{(\mathrm{h} 1)}\left(\operatorname{Tr} \bar{V}_{1}^{\prime}+\operatorname{Tr} \bar{V}_{2}^{\prime \prime}\right), \\
& a_{\left(t_{3}^{\prime}\right)}=-\frac{1}{g_{s}} C_{(\mathrm{h} 2)}\left(\operatorname{Tr} \bar{V}_{1}^{\prime \prime}+\operatorname{Tr} \bar{V}_{2}^{\prime}\right), \\
& a_{\left(t_{1}, t_{2}^{\prime}\right)}=-\frac{1}{g_{s}} C_{(\mathrm{m} 1)}\left(\operatorname{Tr} \bar{V}_{1}^{\prime}+\operatorname{Tr} \bar{V}_{2}^{\prime \prime}\right), \\
& a_{\left(t_{1}, t_{3}^{\prime}\right)}=-\frac{1}{g_{s}} C_{(\mathrm{m} 2)}\left(\operatorname{Tr} \bar{V}_{1}^{\prime \prime}+\operatorname{Tr} \bar{V}_{2}^{\prime}\right), \\
& a_{\left(2 t_{2}^{\prime}\right)}=-\frac{1}{g_{s}} C_{(\mathrm{h} 3)}\left(\operatorname{Tr} \bar{V}_{1}^{\prime 2}+\operatorname{Tr} \bar{V}_{2}^{\prime \prime 2}\right)-\mathrm{i} C_{(\mathrm{h} 5)}\left[\left(\operatorname{Tr} \bar{V}_{1}^{\prime}\right)^{2}+\left(\operatorname{Tr} \bar{V}_{2}^{\prime \prime}\right)^{2}\right], \\
& a_{\left(2 t_{3}^{\prime}\right)}=-\frac{1}{g_{s}} C_{(\mathrm{h} 4)}\left(\operatorname{Tr} \bar{V}_{1}^{\prime \prime 2}+\operatorname{Tr} \bar{V}_{2}^{\prime 2}\right)-\mathrm{i} C_{(\mathrm{h} 6)}\left[\left(\operatorname{Tr} \bar{V}_{1}^{\prime \prime}\right)^{2}+\left(\operatorname{Tr} \bar{V}_{2}^{\prime}\right)^{2}\right], \\
& a_{\left(t_{2}^{\prime}, t_{3}^{\prime}\right)}=-\mathrm{i} C_{(\mathrm{h} 7)}\left(\operatorname{Tr} \bar{V}_{1}^{\prime} \operatorname{Tr} \bar{V}_{2}^{\prime}+\operatorname{Tr} \bar{V}_{1}^{\prime \prime} \operatorname{Tr} \bar{V}_{2}^{\prime \prime}\right), \\
& a_{\left(2 t_{1}, t_{2}^{\prime}\right)}=-\frac{1}{g_{s}}\left(\sum_{k=3}^{6} C_{(\mathrm{m} k)}\right)\left(\operatorname{Tr} \bar{V}_{1}^{\prime}+\operatorname{Tr} \bar{V}_{2}^{\prime \prime}\right) \text {, } \\
& a_{\left(2 t_{1}, t_{3}^{\prime}\right)}=-\frac{1}{g_{s}}\left(\sum_{k=7}^{10} C_{(\mathrm{m} k)}\right)\left(\operatorname{Tr} \bar{V}_{1}^{\prime \prime}+\operatorname{Tr} \bar{V}_{2}^{\prime}\right), \\
& a_{\left(t_{1}, t_{2}, t_{1}^{\prime}\right)}=\frac{1}{g_{s}}\left[\left(C_{(\mathrm{m} 19)}+C_{(\mathrm{m} 20)}\right)\left(\operatorname{Tr} V_{1}^{\prime}+\operatorname{Tr} V_{2}^{\prime \prime}\right)\right. \\
& \left.+\left(C_{(\mathrm{m} 21)}+C_{(\mathrm{m} 22)}\right)\left(\operatorname{Tr} V_{1}^{\prime \prime}+\operatorname{Tr} V_{2}^{\prime}\right)\right] \\
& a_{\left(t_{1}, t_{2}, t_{2}^{\prime}\right)}=-\frac{1}{g_{s}} C_{(\mathrm{m} 11)}\left(\operatorname{Tr} \bar{V}_{1}^{\prime}+\operatorname{Tr} \bar{V}_{2}^{\prime \prime}\right) \text {, } \\
& a_{\left(t_{1}, t_{2}, t_{3}^{\prime}\right)}=-\frac{1}{g_{s}} C_{(\mathrm{m} 12)}\left(\operatorname{Tr} \bar{V}_{1}^{\prime \prime}+\operatorname{Tr} \bar{V}_{2}^{\prime}\right) \text {, } \\
& a_{\left(t_{1}, t_{3}, t_{1}^{\prime}\right)}=\frac{1}{g_{s}}\left[\left(C_{(\mathrm{m} 23)}+C_{(\mathrm{m} 24)}\right)\left(\operatorname{Tr} V_{1}^{\prime}+\operatorname{Tr} V_{2}^{\prime \prime}\right)\right. \\
& \left.+\left(C_{(\mathrm{m} 25)}+C_{(\mathrm{m} 26)}\right)\left(\operatorname{Tr} V_{1}^{\prime \prime}+\operatorname{Tr} V_{2}^{\prime}\right)\right] \\
& a_{\left(t_{1}, t_{3}, t_{2}^{\prime}\right)}=-\frac{1}{g_{s}} C_{(\mathrm{m} 13)}\left(\operatorname{Tr} \bar{V}_{1}^{\prime}+\operatorname{Tr} \bar{V}_{2}^{\prime \prime}\right) \text {, } \\
& a_{\left(t_{1}, t_{3}, t_{3}^{\prime}\right)}=-\frac{1}{g_{s}} C_{(\mathrm{m} 14)}\left(\operatorname{Tr} \bar{V}_{1}^{\prime \prime}+\operatorname{Tr} \bar{V}_{2}^{\prime}\right), \\
& a_{\left(t_{1}, 2 t_{2}^{\prime}\right)}=-\frac{1}{g_{s}} C_{(\mathrm{m} 15)}\left(\operatorname{Tr} \bar{V}_{1}^{\prime 2}+\operatorname{Tr} \bar{V}_{2}^{\prime \prime 2}\right)-\mathrm{i} C_{(\mathrm{m} 16)}\left[\left(\operatorname{Tr} \bar{V}_{1}^{\prime}\right)^{2}+\left(\operatorname{Tr} \bar{V}_{2}^{\prime \prime}\right)^{2}\right], \\
& a_{\left(t_{1}, 2 t_{3}^{\prime}\right)}=-\frac{1}{g_{s}} C_{(\mathrm{m} 17)}\left(\operatorname{Tr} \bar{V}_{1}^{\prime \prime 2}+\operatorname{Tr} \bar{V}_{2}^{\prime 2}\right)-\mathrm{i} C_{(\mathrm{m} 18)}\left[\left(\operatorname{Tr} \bar{V}_{1}^{\prime \prime}\right)^{2}+\left(\operatorname{Tr} \bar{V}_{2}^{\prime}\right)^{2}\right]
\end{aligned}
$$



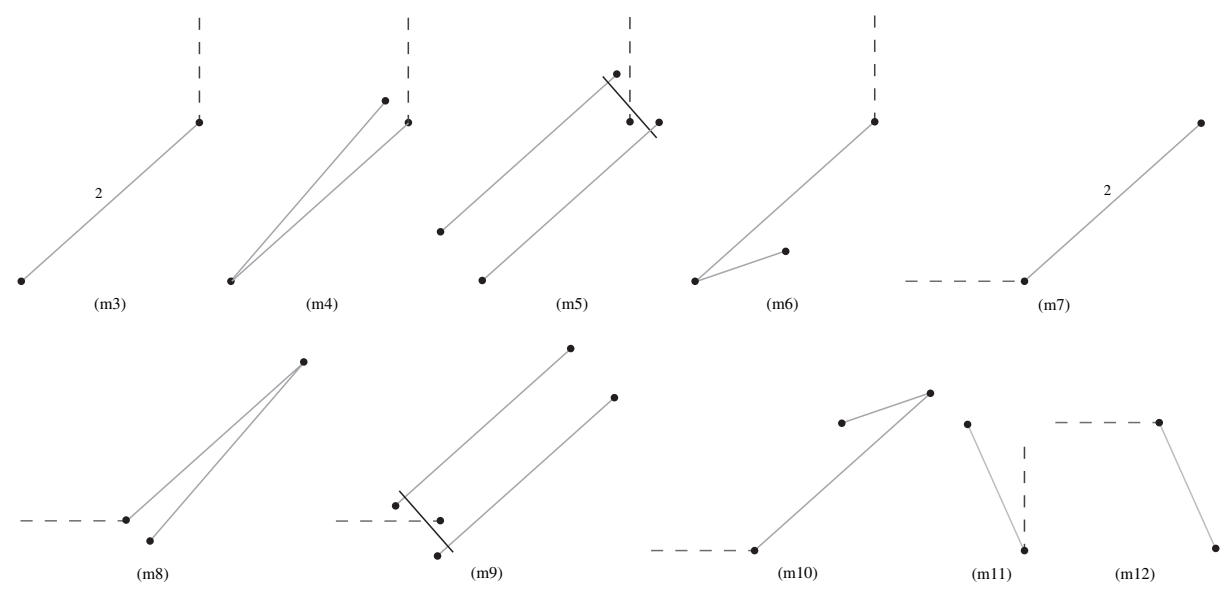

Figure 13: Stable maps: degree 3 mixed classes-I. The color coding is as before.

The expressions for the coefficients $C$ obtained by localization are listed below.

Degree 1:

$$
C_{(\mathrm{h} 1)}=C_{(\mathrm{h} 2)}=-1 .
$$

Degree 2:

$$
\begin{array}{ll}
C_{(\mathrm{h} 3)}=-\frac{z-2}{4 z}, & C_{(\mathrm{h} 4)}=\frac{2 z-1}{4}, \\
C_{(\mathrm{h} 5)}=\frac{z-1}{4 z^{2}}, & C_{(\mathrm{h} 6)}=-\frac{z(z-1)}{4}, \\
C_{(\mathrm{h} 7)}=-1, & C_{(\mathrm{m} 1)}=C_{(\mathrm{m} 2)}=-\frac{6(z-1)^{2}}{z} .
\end{array}
$$

Degree 3:

$$
\begin{array}{ll}
C_{(\mathrm{m} 3)}=\frac{45(z-1)^{2}}{z(z+1)}, & C_{(\mathrm{m} 4)}=C_{(\mathrm{m} 6)}=-\frac{6(z-1)^{2}}{z}, \\
C_{(\mathrm{m} 5)}=-\frac{36(z-1)^{2}}{z^{2}}, & C_{(\mathrm{m} 7)}=\frac{45(z-1)^{2}}{(z+1)}, \\
C_{(\mathrm{m} 9)}=-36(z-1)^{2}, & C_{(\mathrm{m} 8)}=C_{(\mathrm{m} 10)}=-\frac{6(z-1)^{2}}{z}, \\
C_{(\mathrm{m} 11)}=C_{(\mathrm{m} 13)}=3, & C_{(\mathrm{m} 12)}=-\frac{6}{z(z-1)},
\end{array}
$$




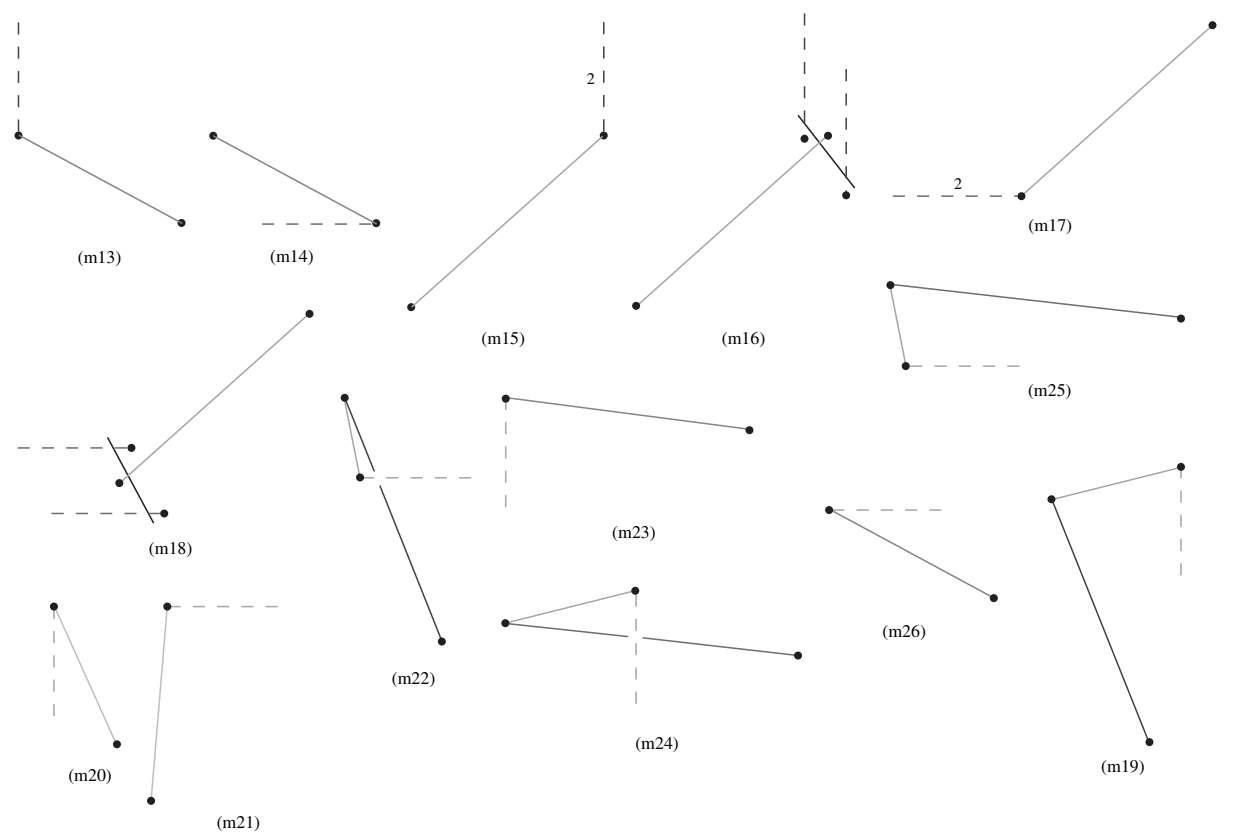

Figure 14: Stable maps: degree 3 mixed classes-II. The color coding is as before.

$$
\begin{aligned}
C_{(\mathrm{m} 14)} & =\frac{6 z^{2}}{z-1}, & C_{(\mathrm{m} 15)} & =\frac{6(z-1)^{2}}{z^{2}}, \\
C_{(\mathrm{m} 16)} & =-\frac{6(z-1)^{2}}{z^{3}}, & C_{(\mathrm{m} 17)} & =6(z-1)^{2}, \\
C_{(\mathrm{m} 18)} & =-6 z(z-1)^{2}, & C_{(\mathrm{m} 19)} & =\frac{6 z^{2}}{z-1}, \\
C_{(\mathrm{m} 20)} & =\frac{12 z^{2}}{z-1}, & C_{(\mathrm{m} 21)} & =-\frac{9(2 z-3)(z-3)}{z-1}, \\
C_{(\mathrm{m} 22)} & =C_{(\mathrm{m} 24)}=0, & C_{(\mathrm{m} 23)} & =\frac{9(3 z-1)(3 z-2)}{z(z-1)}, \\
C_{(\mathrm{m} 25)} & =-\frac{6}{z(z-1)}, & C_{(\mathrm{m} 26)} & =-\frac{12}{z(z-1)} .
\end{aligned}
$$

For the final result, we have to compute the Chern-Simons free energy with all these corrections included. Following the rules given so far, this is a straightforward, although quite tedious computation. In order to simplify this process, we have considered only pure horizontal corrections up to order 2 instead of 3 . The only new aspect in this calculation is that one has to evaluate Chern-Simons expectation values of the form $\left\langle\operatorname{Tr}_{R_{1}}\left(V_{1}^{\prime}\right) \operatorname{Tr}_{R_{2}}\left(\bar{V}_{1}^{\prime}\right)\right\rangle_{p_{1}^{\prime}}$ 
and $\left\langle\operatorname{Tr}_{R_{1}}\left(V_{1}^{\prime}\right) \operatorname{Tr}_{R_{2}}\left(\bar{V}_{1}^{\prime \prime}\right)\right\rangle_{p_{1}^{\prime}, p_{1}^{\prime \prime}}$, where the knots $\Gamma_{1}^{\prime}$ and $\Gamma_{1}^{\prime \prime}$ form a Hopf link with linking number +1 . Such expectation values are typically avoided in local geometric transitions by performing an analytic continuation $[47,52]$. In our situation, this is not possible, hence we have to perform the computations directly. This entails an exercise in representation theory which is discussed in Appendix C. The final result is ${ }^{12}$

$$
\begin{aligned}
g_{\mathrm{s}}^{2} \ln & \left\langle e^{F_{\text {inst }}^{(m, h)}}\right\rangle_{\mid(m, h)} \\
= & -2 q_{2}^{\prime}\left(y-y^{-1}\right)-2 q_{3}^{\prime}\left(y-y^{-1}\right)-\frac{1}{4} q_{2}^{\prime 2}\left(y^{2}-y^{-2}\right)-\frac{1}{4} q_{3}^{\prime 2}\left(y^{2}-y^{-2}\right) \\
& -2 q_{2}^{\prime} q_{3}^{\prime}\left(2 y^{2}-3+y^{-2}\right)+36 q_{1}^{\prime} q_{2}^{\prime}\left(1-y^{-2}\right)+36 q_{1}^{\prime} q_{3}^{\prime}\left(1-y^{-2}\right) \\
& -324 q_{1} q_{1}^{\prime} q_{2}^{\prime}\left(1-y^{-2}\right)-324 q_{1} q_{1}^{\prime} q_{3}^{\prime}\left(1-y^{-2}\right) \\
& +90 q_{1}^{\prime 2} q_{2}^{\prime}\left(5 y-4 y^{-1}-y^{-3}\right) \\
& +90 q_{1}^{\prime 2} q_{3}^{\prime}\left(5 y-4 y^{-1}-y^{-3}\right)-216 q_{1} q_{2} q_{1}^{\prime}\left(y-y^{-1}\right) \\
& -216 q_{1} q_{3} q_{1}^{\prime}\left(y-y^{-1}\right) \\
& +36 q_{1}^{\prime} q_{2}^{\prime} q_{3}^{\prime}\left(3 y-5 y^{-1}+2 y^{-3}\right) \\
& +18 q_{1} q_{2}^{\prime} q_{3}\left(y-y^{-1}\right)+18 q_{1} q_{2} q_{3}^{\prime}\left(y-y^{-1}\right) .
\end{aligned}
$$

Using equation (7.20) supplemented with the extra relations

$$
q_{2}=\frac{\tilde{q}_{2}}{\tilde{q}_{1}}, \quad q_{3}=\frac{\tilde{q}_{3}}{\tilde{q}_{1}}, \quad q_{2}^{\prime}=\left(\tilde{q}_{1}\right)^{1 / 2} \tilde{q}_{2}, \quad q_{3}^{\prime}=\left(\tilde{q}_{1}\right)^{1 / 2} \tilde{q}_{3},
$$

we find the following expression in terms of closed string variables

$$
\begin{aligned}
g_{\mathrm{s}}^{2} \ln & \left\langle e^{F_{\text {inst }}^{(m, h)}}\right\rangle_{\mid(m, h)} \\
= & 2\left(\tilde{q}_{2}+\tilde{q}_{3}\right)-2\left(\tilde{q}_{1} \tilde{q}_{2}+\tilde{q}_{1} \tilde{q}_{3}+\tilde{q}_{2} \tilde{q}_{3}\right)+\frac{1}{4}\left(\tilde{q}_{2}^{2}+\tilde{q}_{3}^{2}\right)+6 \tilde{q}_{1} \tilde{q}_{2} \tilde{q}_{3} \\
& -\frac{1}{4}\left(\tilde{q}_{1}^{2} \tilde{q}_{2}^{2}+\tilde{q}_{1}^{2} \tilde{q}_{3}^{2}\right)-4 \tilde{q}_{1}^{2} \tilde{q}_{2} \tilde{q}_{3}-36\left(\tilde{q}_{2}+\tilde{q}_{3}\right) \tilde{q}_{4}+126\left(\tilde{q}_{2}+\tilde{q}_{3}\right) \tilde{q}_{4}^{2} \\
& +36\left(\tilde{q}_{1} \tilde{q}_{2}+\tilde{q}_{1} \tilde{q}_{3}+\tilde{q}_{2} \tilde{q}_{3}\right) \tilde{q}_{4}-252 \tilde{q}_{1}\left(\tilde{q}_{2}+\tilde{q}_{3}\right) \tilde{q}_{4}^{2}-144 \tilde{q}_{1} \tilde{q}_{2} \tilde{q}_{3} \tilde{q}_{4} \\
& +126 \tilde{q}_{1}^{2}\left(\tilde{q}_{2}+\tilde{q}_{3}\right) \tilde{q}_{4}^{2}+108 \tilde{q}_{1}^{2} \tilde{q}_{2} \tilde{q}_{3} \tilde{q}_{4} .
\end{aligned}
$$

The contribution of the pure Chern-Simons sector reads

$$
\mathcal{F}_{\mathrm{CS}, 1}^{(0)}+\mathcal{F}_{\mathrm{CS}, 2}^{(0)}=y_{1}^{2}+y_{2}^{2}+\frac{1}{8}\left(y_{1}^{4}+y_{2}^{4}\right)+\frac{1}{27}\left(y_{1}^{6}+y_{2}^{6}\right)+\cdots,
$$

\footnotetext{
${ }^{12}$ The subscript $\mid(m, h)$ means that we only keep mixed and horizontal terms in the final answer. The pure vertical terms have been already taken into account in equation (7.33).
} 
which in closed string variables becomes

$$
\mathcal{F}_{\mathrm{CS}, 1}^{(0)}+\mathcal{F}_{\mathrm{CS}, 2}^{(0)}=2 \tilde{q}_{1}+\frac{1}{4} \tilde{q}_{1}^{2}+\frac{2}{27} \tilde{q}_{1}^{3}+\cdots
$$

The full open closed expansion of the topological free energy is obtained by adding equations (7.33), (7.41), (7.42), and the genus zero closed string contribution (A.15) expressed in terms of closed string variables $\tilde{q}_{1}, \ldots, \tilde{q}_{4}$. This gives the following expression

$$
\begin{aligned}
\mathcal{F}_{Y ; \mathrm{cl}}^{(0) ; \text { inst }}+\mathcal{F}_{\mathrm{CS}, 1}^{(0)}+\mathcal{F}_{\mathrm{CS}, 2}^{(0)}+g_{s}^{2} \ln \left\langle e^{F_{\text {inst }}^{(f)}}\right\rangle+g_{s}^{2} \ln \left\langle e^{F_{\text {inst }}^{(m, h)}}\right\rangle_{\mid(m, h)} \\
=2\left(\tilde{q}_{1}+\tilde{q}_{2}+\tilde{q}_{3}\right)+36 \tilde{q}_{4}-2\left(\tilde{q}_{1} \tilde{q}_{2}+\tilde{q}_{1} \tilde{q}_{3}+\tilde{q}_{2} \tilde{q}_{3}\right)+126\left(\tilde{q}_{1}+\tilde{q}_{2}+\tilde{q}_{3}\right) \tilde{q}_{4} \\
\quad+\frac{9}{2} \tilde{q}_{4}^{2}+\frac{1}{4}\left(\tilde{q}_{1}^{2}+\tilde{q}_{2}^{2}+\tilde{q}_{3}^{2}\right)-\frac{1}{4}\left(\tilde{q}_{1}^{2} \tilde{q}_{2}^{2}+\tilde{q}_{1}^{2} \tilde{q}_{3}^{2}\right)+\frac{4}{3} \tilde{q}_{4}^{3}+126\left(\tilde{q}_{1}+\tilde{q}_{2}+\tilde{q}_{3}\right) \tilde{q}_{4}^{2} \\
\quad+36\left(\tilde{q}_{1} \tilde{q}_{2}+\tilde{q}_{1} \tilde{q}_{3}+\tilde{q}_{2} \tilde{q}_{3}\right) \tilde{q}_{4}+6 \tilde{q}_{1} \tilde{q}_{2} \tilde{q}_{3}+\frac{2}{27} \tilde{q}_{1}^{3}+2 \tilde{q}_{1} \tilde{q}_{4}^{3}+\frac{207}{4} \tilde{q}_{1}^{2} \tilde{q}_{4}^{2} \\
\quad+2178\left(\tilde{q}_{1} \tilde{q}_{2}+\tilde{q}_{1} \tilde{q}_{3}\right) \tilde{q}_{4}^{2}-144 \tilde{q}_{1} \tilde{q}_{2} \tilde{q}_{3} \tilde{q}_{4} \\
\quad-4 \tilde{q}_{1}^{2} \tilde{q}_{2} \tilde{q}_{3}+152 \tilde{q}_{1}^{2} \tilde{q}_{4}^{3}+126\left(\tilde{q}_{1}^{2} \tilde{q}_{2}+\tilde{q}_{1}^{2} \tilde{q}_{3}\right) \tilde{q}_{4}^{2}+108 \tilde{q}_{1}^{2} \tilde{q}_{2} \tilde{q}_{3} \tilde{q}_{4}+\frac{20}{3} \tilde{q}_{1}^{3} \tilde{q}_{4}^{3} .
\end{aligned}
$$

This result should be compared to the closed string expansion (A.14), keeping carefully track of the degrees of the terms in the two formulae. It can be easily seen that we obtain an exact agreement, except for the following terms in the closed string formula (A.14)

$$
\begin{aligned}
& \frac{2}{27}\left(\tilde{q}_{2}^{3}+\tilde{q}_{3}^{3}\right)+2\left(\tilde{q}_{2}+\tilde{q}_{3}\right) \tilde{q}_{4}^{3}+\frac{207}{4}\left(\tilde{q}_{2}^{2}+\tilde{q}_{3}^{2}\right) \tilde{q}_{4}^{2}+2178 \tilde{q}_{2} \tilde{q}_{3} \tilde{q}_{4}^{2} \\
& \quad-4 \tilde{q}_{1} \tilde{q}_{2} \tilde{q}_{3}\left(\tilde{q}_{2}+\tilde{q}_{3}\right)+152\left(\tilde{q}_{2}^{2}+\tilde{q}_{3}^{2}\right) \tilde{q}_{4}^{3}+126\left(\tilde{q}_{1} \tilde{q}_{2}^{2}+\tilde{q}_{1} \tilde{q}_{3}^{2}+\tilde{q}_{2}^{2} \tilde{q}_{3}+\tilde{q}_{2} \tilde{q}_{3}^{2}\right) \tilde{q}_{4}^{2} \\
& \quad+108\left(\tilde{q}_{1} \tilde{q}_{2}^{2} \tilde{q}_{3}+\tilde{q}_{1} \tilde{q}_{2} \tilde{q}_{3}^{2}\right) \tilde{q}_{4}+\frac{20}{3}\left(\tilde{q}_{2}^{3}+\tilde{q}_{3}^{3}\right) \tilde{q}_{4}^{3} .
\end{aligned}
$$

These are either pure horizontal terms of degree 3 (the first two terms) or mixed and vertical terms of degree at least 4 . Such terms cannot be obtained in the open string expansion unless we include higher degree corrections in the open string instanton series. (For example, we have checked that the first two terms in equation (7.45) are recovered if we include degree 3 pure horizontal corrections in equation (7.34). Therefore, we can conclude that there is a perfect agreement, once the degrees are matched consistently. This is a very convincing evidence for the duality conjecture, and of the open string techniques developed in this paper. 


\section{Appendix A Genus zero closed string partition functions}

In this section, we will compute the genus zero closed string partition functions of $\widetilde{Y}$ and $Y$ using mirror symmetry. First, we determine the Kähler and Mori cones of the ambient toric varieties and then we write down and solve the GKZ systems. From this, we will determine the Gromov-Witten invariants of the Calabi-Yau hypersurfaces.

\section{A.1 The Kähler and Mori cones of $Z$ and $\widetilde{Z}$}

Following the method of piecewise linear functions of ref. [51], we obtain the following basis for the Mori cone of $\mathbb{P}_{\nabla_{Y}}$

$$
\begin{array}{lccccccc}
l^{(1)}= & \zeta_{1} & \zeta_{2} & \zeta_{3} & \zeta_{4} & \zeta_{5} & \zeta_{6} & \zeta_{7} \\
l^{(2)}= & 0 & 1 & 0 & 0 & 1 & 1 \\
l^{(3)}= & 0 & 1 & -1 & 0 & 1 & -1 & 0 \\
1 & 0 & -1 & 1 & 0 & -1 & 0
\end{array},
$$

where $\zeta_{i}, i=1, \ldots, 7$, are the divisor classes corresponding, respectively, to the $i$ th vertex of $\nabla_{Y}$. Therefore, the Kähler cone of $\mathbb{P}_{\nabla_{Y}}$ is generated by the divisor classes $\zeta_{1}, \zeta_{2}$ and $\zeta_{7}$. The only nonvanishing intersection numbers in the Calabi-Yau hypersurface $Y$ are

$$
\zeta_{7}^{3}=18, \quad \zeta_{7}^{2} \zeta_{1}=\zeta_{7}^{2} \zeta_{2}=6, \quad \zeta_{1} \zeta_{2} \zeta_{7}=3 .
$$

Similarly, the basis of the Mori cone of $\mathbb{P}_{\nabla_{\tilde{Y}}}$ is given by

$$
\begin{array}{lcccccccc}
\tilde{l}^{(1)}= & \tilde{\zeta}_{1} & \tilde{\zeta}_{2} & \tilde{\zeta}_{3} & \tilde{\zeta}_{4} & \tilde{\zeta}_{5} & \tilde{\zeta}_{6} & \tilde{\zeta}_{7} & \tilde{\zeta}_{8} \\
\tilde{l}^{(2)}= & 0 & 1 & 0 & 0 & 1 & 0 & -1 \\
\tilde{l}^{(3)}= & 1 & 0 & 0 & 1 & 0 & 0 & -1 \\
\tilde{l}^{(4)}= & 0 & 0 & 1 & 0 & 0 & 0 & -1 \\
0 & 0 & 0 & 0 & 0 & 0 & 1 & 1
\end{array},
$$

where $\tilde{\zeta}_{i}, i=1, \ldots, 8$, are the divisor classes corresponding, respectively, to the $i$ th vertex of $\nabla_{\widetilde{Y}}$. Then the Kähler cone of $\mathbb{P}_{\nabla_{\tilde{Y}}}$ is generated by the divisor classes $\tilde{\zeta}_{4}, \tilde{\zeta}_{5}, \tilde{\zeta}_{6}$ and $\tilde{\zeta}_{7}$. The triple intersections can be easily computed using SCHUBERT [34]. The nonvanishing intersection numbers in the Calabi-Yau hypersurface $\widetilde{Y}$ are

$$
\begin{aligned}
& \tilde{\zeta}_{7}^{3}=18, \quad \tilde{\zeta}_{4} \tilde{\zeta}_{7}^{2}=6, \quad \tilde{\zeta}_{5} \tilde{\zeta}_{7}^{2}=6, \quad \tilde{\zeta}_{6} \tilde{\zeta}_{7}^{2}=6, \\
& \tilde{\zeta}_{4} \tilde{\zeta}_{5} \tilde{\zeta}_{7}=3, \quad \tilde{\zeta}_{4} \tilde{\zeta}_{6} \tilde{\zeta}_{7}=3, \quad \tilde{\zeta}_{5} \tilde{\zeta}_{6} \tilde{\zeta}_{7}=3, \quad \tilde{\zeta}_{4} \tilde{\zeta}_{5} \tilde{\zeta}_{6}=2 .
\end{aligned}
$$

We also have $c_{2}(\tilde{Y}) \tilde{\zeta}_{7}=72$ and $c_{2}(\tilde{Y}) \tilde{\zeta}_{4}=c_{2}(\tilde{Y}) \tilde{\zeta}_{5}=c_{2}(\tilde{Y}) \tilde{\zeta}_{6}=24$. 


\section{A.2 The GKZ operators and the prepotential}

The GKZ operators associated with the Mori cone generators (A.3) read

$$
\begin{aligned}
\widetilde{\mathcal{D}}_{i} & =\widetilde{\Theta}_{i}^{2}-\tilde{z}_{i}\left(\widetilde{\Theta}_{4}-\widetilde{\Theta}_{1}-\widetilde{\Theta}_{2}-\widetilde{\Theta}_{3}\right)\left(2 \widetilde{\Theta}_{4}+\widetilde{\Theta}_{1}+\widetilde{\Theta}_{2}+\widetilde{\Theta}_{3}+1\right), \\
i & =1, \ldots, 3, \\
\widetilde{\mathcal{D}}_{4} & =\widetilde{\Theta}_{4}\left(\widetilde{\Theta}_{4}-\widetilde{\Theta}_{1}-\widetilde{\Theta}_{2}-\widetilde{\Theta}_{3}\right)-\tilde{z}_{4} \prod_{i=1}^{2}\left(2 \widetilde{\Theta}_{4}+\widetilde{\Theta}_{1}+\widetilde{\Theta}_{2}+\widetilde{\Theta}_{3}+i\right),
\end{aligned}
$$

where $\tilde{z}_{i}, i=1, \ldots, 4$, are the large complex structure coordinates and $\widetilde{\Theta}_{i}=$ $\tilde{z}_{i} \partial / \partial \tilde{z}_{i}, i=1, \ldots, 4$. Note that the GKZ operators are sufficient to determine a complete set of period integrals for the mirror of $\widetilde{Y}$ since $\nabla_{\widetilde{Y}}$ does not have any points interior to codimension 1 faces and admits a (unique) maximal triangulation with all the cones of unit volume $[27,28]$. Also note that, as a consistency check, it is possible to rederive the intersection numbers (A.4) starting from the principal part of the GKZ operators (A.5).

We denote by $\varpi_{0}$ the solution of equation (A.5) that is analytic at $\tilde{z}_{i}=0$, $i=1, \ldots, 4$. This solution is given by the series $[11,27]$

$$
\begin{aligned}
\varpi_{0} & =\sum_{n_{1}, n_{2}, n_{3}, n_{4}} f_{0} \\
& =\sum_{n_{1}, n_{2}, n_{3}, n_{4}} \frac{\tilde{z}_{1}^{n_{1}} \tilde{z}_{2}^{n_{2}} \tilde{z}_{3}^{n_{3}} \tilde{z}_{4}^{n_{4}} \Gamma\left(1+n_{1}+n_{2}+n_{3}+2 n_{4}\right)}{\Gamma\left(1+n_{1}\right)^{2} \Gamma\left(1+n_{2}\right)^{2} \Gamma\left(1+n_{3}\right)^{2} \Gamma\left(1+n_{4}\right) \Gamma\left(1-n_{1}-n_{2}-n_{3}+n_{4}\right)} .
\end{aligned}
$$

There are four solutions of equation (A.5) that are asymptotically like $\ln \tilde{z}_{i}$, $i=1, \ldots, 4$

$$
\varpi_{i}=\varpi_{0} \ln \tilde{z}_{i}+f_{i},
$$

where

$$
\begin{aligned}
f_{i} & =\sum_{n_{1}, n_{2}, n_{3}, n_{4}}\left[f_{0}\left(S_{n_{1}+n_{2}+n_{3}+2 n_{4}}-2 S_{n_{i}}+S_{-n_{1}-n_{2}-n_{3}+n_{4}}\right)+h\right], \\
i & =1, \ldots, 3, \\
f_{4} & =\sum_{n_{1}, n_{2}, n_{3}, n_{4}}\left[f_{0}\left(2 S_{n_{1}+n_{2}+n_{3}+2 n_{4}}-S_{n_{4}}-S_{-n_{1}-n_{2}-n_{3}+n_{4}}\right)-h\right] .
\end{aligned}
$$

In the above, we have introduced the notation $S_{n}=\sum_{k=1}^{n} 1 / k$ and we have defined

$$
h=\frac{\left(-\tilde{z}_{1}\right)^{n_{1}}\left(-\tilde{z}_{2}\right)^{n_{2}}\left(-\tilde{z}_{3}\right)^{n_{3}}\left(-\tilde{z}_{4}\right)^{n_{4}} \Gamma\left(1+n_{1}+n_{2}+n_{3}+2 n_{4}\right) \Gamma\left(n_{1}+n_{2}+n_{3}-n_{4}\right)}{\Gamma\left(1+n_{1}\right)^{2} \Gamma\left(1+n_{2}\right)^{2} \Gamma\left(1+n_{3}\right)^{2} \Gamma\left(1+n_{4}\right)} .
$$

Following refs. [11,27], we can now write down the mirror map

$$
\tilde{t}_{i}=-\frac{\varpi_{i}}{\varpi_{0}}=-\ln \tilde{z}_{i}-\frac{f_{i}}{\varpi_{0}}, \quad i=1, \ldots, 4 .
$$


In order to compute the genus zero Gromov-Witten invariants of $\tilde{Y}$, we need to find the second-order solutions of the system of differential equations (A.5). These are easily obtained to be given by

$$
\begin{aligned}
& \varpi_{i j}=\varpi_{0} \ln \tilde{z}_{i} \ln \tilde{z}_{j}+\ln \tilde{z}_{i} f_{j}+\ln \tilde{z}_{j} f_{i}+f_{i j}, \quad i, j=1, \ldots, 3, \quad i \neq j, \\
& \varpi_{i 4}=\varpi_{0} \ln \tilde{z}_{i} \ln \tilde{z}_{4}+\ln \tilde{z}_{i} f_{4}+\ln \tilde{z}_{4} f_{i}+f_{i 4}, \quad i=1, \ldots, 3, \\
& \varpi_{44}=\varpi_{0}\left(\ln \tilde{z}_{4}\right)^{2}+2 \ln \tilde{z}_{4} f_{4}+f_{44},
\end{aligned}
$$

where

$$
\begin{aligned}
& f_{i j}=\sum_{n_{1}, n_{2}, n_{3}, n_{4}}\left[f _ { 0 } \left(S_{n_{1}+n_{2}+n_{3}+2 n_{4}}^{2}\right.\right. \\
& -S_{\left(n_{1}+n_{2}+n_{3}+2 n_{4}\right)^{2}}-2 S_{-n_{1}-n_{2}-n_{3}+n_{4}}\left(S_{n_{i}}+S_{n_{j}}\right) \\
& +2 S_{n_{1}+n_{2}+n_{3}+2 n_{4}}\left(S_{-n_{1}-n_{2}-n_{3}+n_{4}}-S_{n_{i}}-S_{n_{j}}\right) \\
& \left.+S_{-n_{1}-n_{2}-n_{3}+n_{4}}^{2}+S_{\left(-n_{1}-n_{2}-n_{3}+n_{4}\right)^{2}}+4 S_{n_{i}} S_{n_{j}}\right)+2 S_{\left(n_{i}\right)}^{2} \delta_{i j}\left(f_{0}\right) \\
& \left.+2 h\left(S_{n_{1}+n_{2}+n_{3}-n_{4}-1}+S_{n_{1}+n_{2}+n_{3}+2 n_{4}}-S_{n_{i}}-S_{n_{j}}\right)\right], \\
& i, j=1, \ldots, 3, \quad i \neq j \text {, } \\
& f_{i 4}=\sum_{n_{1}, n_{2}, n_{3}, n_{4}}\left[f _ { 0 } \left(2 S_{n_{1}+n_{2}+n_{3}+2 n_{4}}^{2}-2 S_{\left(n_{1}+n_{2}+n_{3}+2 n_{4}\right)^{2}}\right.\right. \\
& +S_{-n_{1}-n_{2}-n_{3}+n_{4}}\left(2 S_{n_{i}}-S_{n_{4}}\right) \\
& +S_{n_{1}+n_{2}+n_{3}+2 n_{4}}\left(S_{-n_{1}-n_{2}-n_{3}+n_{4}}-4 S_{n_{i}}-S_{n_{4}}\right) \\
& \left.-S_{-n_{1}-n_{2}-n_{3}+n_{4}}^{2}-S_{\left(-n_{1}-n_{2}-n_{3}+n_{4}\right)^{2}}+2 S_{n_{i}} S_{n_{4}}\right) \\
& \left.+h\left(-2 S_{n_{1}+n_{2}+n_{3}-n_{4}-1}+S_{n_{1}+n_{2}+n_{3}+2 n_{4}}+2 S_{n_{i}}-S_{n_{4}}\right)\right] \text {, } \\
& i=1, \ldots, 3 \text {, } \\
& f_{44}=\sum_{n_{1}, n_{2}, n_{3}, n_{4}}\left[f _ { 0 } \left(4 S_{n_{1}+n_{2}+n_{3}+2 n_{4}}^{2}-4 S_{\left(n_{1}+n_{2}+n_{3}+2 n_{4}\right)^{2}}\right.\right. \\
& +2 S_{n_{4}} S_{-n_{1}-n_{2}-n_{3}+n_{4}}+S_{n_{4}}^{2}+S_{\left(n_{4}\right)^{2}} \\
& -4 S_{n_{1}+n_{2}+n_{3}+2 n_{4}}\left(S_{n_{4}}+S_{-n_{1}-n_{2}-n_{3}+n_{4}}\right) \\
& \left.+S_{-n_{1}-n_{2}-n_{3}+n_{4}}^{2}+S_{\left(-n_{1}-n_{2}-n_{3}+n_{4}\right)^{2}}\right) \\
& \left.+2 h\left(S_{n_{1}+n_{2}+n_{3}-n_{4}-1}-2 S_{n_{1}+n_{2}+n_{3}+2 n_{4}}+S_{n_{4}}\right)\right] \text {. }
\end{aligned}
$$

In equation (A.12), we have introduced the notation $S_{(n)^{2}}=\sum_{k=1}^{n} 1 / k^{2}$. 
Taking into account the triple intersections (A.4), a consequence of mirror symmetry is that the following equations hold true $[11,27,28]$

$$
\begin{aligned}
& \partial_{\tilde{t}_{i}} \mathcal{F}_{\widetilde{Y} ; \mathrm{cl}}^{(0)}=-\frac{1}{\varpi_{0}}\left[2 \varpi_{j k}+3 \sum_{j=1}^{3} \varpi_{j 4}+3 \varpi_{44}\right], \quad i=1, \ldots, 3, \quad j, k \neq i, 4, \\
& \partial_{\tilde{t}_{4}} \mathcal{F}_{\widetilde{Y} ; \mathrm{cl}}^{(0)}=-\frac{3}{\varpi_{0}}\left[\sum_{\substack{i, j, k=1 \\
i<j<k}}^{3} \varpi_{i j}+2 \sum_{i=1}^{3} \varpi_{i 4}+3 \varpi_{44}\right]
\end{aligned}
$$

The system of equations (A.13) completely determines the prepotential. Up to degree 6 in the instanton expansion, ${ }^{13}$ the prepotential for $\tilde{Y}$ reads

$$
\begin{aligned}
\mathcal{F}_{\tilde{Y} ; \mathrm{cl}}^{(0)}= & -3 \tilde{t}_{4}^{3}-3\left(\tilde{t}_{1}+\tilde{t}_{2}+\tilde{t}_{3}\right) \tilde{t}_{4}^{2}-3\left(\tilde{t}_{1} \tilde{t}_{2}+\tilde{t}_{1} \tilde{t}_{3}+\tilde{t}_{2} \tilde{t}_{3}\right) \tilde{t}_{4}-2 \tilde{t}_{1} \tilde{t}_{2} \tilde{t}_{3} \\
& +\tilde{t}_{1}+\tilde{t}_{2}+\tilde{t}_{3}+3 \tilde{t}_{4}+2\left(\tilde{q}_{1}+\tilde{q}_{2}+\tilde{q}_{3}\right)+36 \tilde{q}_{4}-2\left(\tilde{q}_{1} \tilde{q}_{2}+\tilde{q}_{1} \tilde{q}_{3}+\tilde{q}_{2} \tilde{q}_{3}\right) \\
& +126\left(\tilde{q}_{1}+\tilde{q}_{2}+\tilde{q}_{3}\right) \tilde{q}_{4}+\frac{9}{2} \tilde{q}_{4}^{2}+\frac{1}{4}\left(\tilde{q}_{1}^{2}+\tilde{q}_{2}^{2}+\tilde{q}_{3}^{2}\right)+\frac{4}{3} \tilde{q}_{4}^{3} \\
& +126\left(\tilde{q}_{1}+\tilde{q}_{2}+\tilde{q}_{3}\right) \tilde{q}_{4}^{2}+36\left(\tilde{q}_{1} \tilde{q}_{2}+\tilde{q}_{1} \tilde{q}_{3}+\tilde{q}_{2} \tilde{q}_{3}\right) \tilde{q}_{4}+6 \tilde{q}_{1} \tilde{q}_{2} \tilde{q}_{3} \\
& +\frac{2}{27}\left(\tilde{q}_{1}^{3}+\tilde{q}_{2}^{3}+\tilde{q}_{3}^{3}\right)+2\left(\tilde{q}_{1}+\tilde{q}_{2}+\tilde{q}_{3}\right) \tilde{q}_{4}^{3}-\frac{1}{4}\left(\tilde{q}_{1}^{2} \tilde{q}_{2}^{2}+\tilde{q}_{1}^{2} \tilde{q}_{3}^{2}+\tilde{q}_{2}^{2} \tilde{q}_{3}^{2}\right) \\
& +\frac{207}{4}\left(\tilde{q}_{1}^{2}+\tilde{q}_{2}^{2}+\tilde{q}_{3}^{2}\right) \tilde{q}_{4}^{2}+2178\left(\tilde{q}_{1} \tilde{q}_{2}+\tilde{q}_{1} \tilde{q}_{3}+\tilde{q}_{2} \tilde{q}_{3}\right) \tilde{q}_{4}^{2}-144 \tilde{q}_{1} \tilde{q}_{2} \tilde{q}_{3} \tilde{q}_{4} \\
& -4 \tilde{q}_{1} \tilde{q}_{2} \tilde{q}_{3}\left(\tilde{q}_{1}+\tilde{q}_{2}+\tilde{q}_{3}\right)+152\left(\tilde{q}_{1}^{2}+\tilde{q}_{2}^{2}+\tilde{q}_{3}^{2}\right) \tilde{q}_{4}^{3} \\
& +126\left(\tilde{q}_{1}^{2} \tilde{q}_{2}+\tilde{q}_{1} \tilde{q}_{2}^{2}+\tilde{q}_{1}^{2} \tilde{q}_{3}+\tilde{q}_{1} \tilde{q}_{3}^{2}+\tilde{q}_{2}^{2} \tilde{q}_{3}+\tilde{q}_{2} \tilde{q}_{3}^{2}\right) \tilde{q}_{4}^{2} \\
& +108\left(\tilde{q}_{1}^{2} \tilde{q}_{2} \tilde{q}_{3}+\tilde{q}_{1} \tilde{q}_{2}^{2} \tilde{q}_{3}+\tilde{q}_{1} \tilde{q}_{2} \tilde{q}_{3}^{2}\right) \tilde{q}_{4}+\frac{20}{3}\left(\tilde{q}_{1}^{3}+\tilde{q}_{2}^{3}+\tilde{q}_{3}^{3}\right) \tilde{q}_{4}^{3}+\cdots
\end{aligned}
$$

where $\tilde{q}_{i}=\mathrm{e}^{-\tilde{t}_{i}}, i=1, \ldots, 4$.

Proceeding in a similar manner, we obtain the prepotential for $Y$ up to degree 3 in the instanton expansion

$$
\begin{aligned}
\mathcal{F}_{Y ; \mathrm{cl}}^{(0)}= & -3 t_{1}^{3}-3 t_{1}^{2} t_{2}-3 t_{1}^{2} t_{3}-3 t_{1} t_{2} t_{3}+3 t_{1}+t_{2}+t_{3}+162 q_{1} \\
& +\frac{729}{4} q_{1}^{2}+162\left(q_{1} q_{2}+q_{1} q_{3}\right)+162 q_{1}^{3}+2430\left(q_{1}^{2} q_{2}+q_{1}^{2} q_{3}\right)+\cdots
\end{aligned}
$$

where $q_{i}=\mathrm{e}^{-t_{i}}, i=1, \ldots, 3$.

\footnotetext{
${ }^{13}$ We only include terms that correspond to instanton corrections of degree less or equal than 3 on $Y$.
} 


\section{Appendix B Disc multicovers}

Here we summarize the computation of disc multicover contributions using the convex obstruction bundle approach. As explained in Section 6, it suffices to consider only the discs $D_{1}^{\prime}$ and $D_{3}^{\prime}$ since all other cases are similar.

\section{B.1 Multicovers of $D_{1}^{\prime}$}

Recall that the disc $D_{1}^{\prime}$ is obtained by intersecting the curve $C_{13}^{\prime}$ with the 3-cycle $\bar{L}_{1}$, according to the local analysis of Section 6.2. The defining equations of $D_{1}^{\prime}$ in the coordinate patches $\mathcal{U}_{1}=\left\{Z_{2} \neq 0, Z_{4} \neq 0, W \neq 0\right\}$ and $\mathcal{U}_{1}^{\prime}=\left\{Z_{2} \neq 0, Z_{4} \neq 0, V \neq 0\right\}$ are

$$
\begin{aligned}
& \mathcal{U}_{1}: \quad x_{1}=u_{1}=0, \quad y_{1} v_{1}=\mu, \quad\left|y_{1}\right| \leq \mu^{1 / 2}, \quad\left|v_{1}\right| \geq \mu^{1 / 2} \\
& \mathcal{U}_{1}^{\prime}: \quad x_{1}^{\prime}=u_{1}^{\prime}=0, \quad y_{1}^{\prime}=\mu v_{1}^{\prime}, \quad\left|y_{1}^{\prime}\right| \leq \mu^{1 / 2}, \quad\left|v_{1}^{\prime}\right| \leq \mu^{-1 / 2} \text {. }
\end{aligned}
$$

We have to compute the cohomology groups $H^{0}(\Delta, \mathcal{T})$ and $H^{1}(\Delta, \mathcal{T})$ and the obstruction groups $H^{0}(\Delta, \mathcal{L})$ and $H^{1}(\Delta, \mathcal{L})$ defined in Section 6.3. The computation is performed in Cech cohomology, as in refs. [16,45]. We will work with the following two set cover of the domain $\Delta$

$$
\Upsilon_{1}^{\prime}=\left\{0 \leq\left|t^{\prime}\right|<\mu^{-1 / 2 d_{1}^{\prime}}\right\}, \quad \Upsilon_{1}=\left\{\mu^{1 / 2 d_{1}^{\prime}} \leq|t|<\left(\mu+\epsilon^{2}\right)^{1 / 2 d_{1}^{\prime}}\right\}
$$

A Galois cover of degree $d_{1}^{\prime}$ of $D_{1}^{\prime}$ is given in local coordinates by

$$
\begin{aligned}
& \mathcal{U}_{1}^{\prime}: \quad x_{1}^{\prime}\left(t^{\prime}\right)=0, \quad y_{1}^{\prime}\left(t^{\prime}\right)=\mu t^{\prime d_{1}^{\prime}}, \quad u_{1}^{\prime}\left(t^{\prime}\right)=0, \quad v_{1}^{\prime}\left(t^{\prime}\right)=t^{\prime d_{1}^{\prime}} \\
& \mathcal{U}_{1}: \quad x_{1}(t)=0, \quad y_{1}(t)=\frac{\mu}{t^{d_{1}^{\prime}}}, \quad u_{1}(t)=0, \quad v_{1}(t)=t^{d_{1}^{\prime}} .
\end{aligned}
$$

Proceeding as in ref. [16], we construct the Čech complex

$$
0 \longrightarrow \mathcal{T}_{\Delta}\left(\Upsilon_{1}^{\prime}\right) \oplus \mathcal{T}_{\Delta}\left(\Upsilon_{1}\right) \stackrel{\kappa}{\longrightarrow} \mathcal{T}_{\Delta}\left(\Upsilon_{1}^{\prime} \cap \Upsilon_{1}\right) \longrightarrow 0
$$


where the generic sections in $\mathcal{T}_{\Delta}\left(\Upsilon_{1}^{\prime}\right)$ and $\mathcal{T}_{\Delta}\left(\Upsilon_{1}\right)$ can be written as

$$
\begin{aligned}
s_{1}^{\prime}= & \left(\sum_{n=0}^{\infty} \alpha_{n}^{\prime} t^{\prime n}\right) \partial_{x_{1}^{\prime}}+\left(\mu \sum_{n=0}^{\infty} \beta_{n}^{\prime} t^{\prime n}\right) \partial_{y_{1}^{\prime}}+\left(\sum_{n=0}^{\infty} \gamma_{n}^{\prime} t^{\prime n}\right) \partial_{u_{1}^{\prime}} \\
& +\left(\sum_{n=0}^{\infty} \delta_{n}^{\prime} t^{\prime n}\right) \partial_{v_{1}^{\prime}} \\
s_{1}= & \left(\sum_{n \in \mathbb{Z}} \alpha_{n} t^{n}\right) \partial_{x_{1}}+\left(\mu \sum_{n \in \mathbb{Z}} \beta_{n} t^{n}\right) \partial_{y_{1}}+\left(\sum_{n \in \mathbb{Z}} \gamma_{n} t^{n}\right) \partial_{u_{1}} . \\
& +\left(\sum_{n \in \mathbb{Z}} \delta_{n} t^{n}\right) \partial_{v_{1}}
\end{aligned}
$$

Note that we sum over $n \geq 0$ for sections in $\mathcal{T}_{\Delta}\left(\Upsilon_{1}^{\prime}\right)$. In order to have a uniform notation, we can extend these sums to $n \in \mathbb{Z}$, with the convention that $\alpha_{n}^{\prime}, \ldots, \delta_{n}^{\prime}$ are zero for $n<0$. The boundary conditions at $|t|=\mu^{1 / 2 d_{1}^{\prime}}$ yield the following relations between the coefficients

$$
\alpha_{n}=\mu^{-n / d_{1}^{\prime}} \bar{\gamma}_{-n}, \quad \beta_{n}=\mu^{-\left(d_{1}^{\prime}+n\right) / d_{1}^{\prime}} \bar{\delta}_{-n} .
$$

Now we have to compute the kernel and cokernel of $\kappa$. Using the linear transformations

$$
\partial_{x_{1}}=\partial_{x_{1}^{\prime}}, \quad \partial_{y_{1}}=\partial_{y_{1}^{\prime}}, \quad \partial_{u_{1}}=v_{1}^{\prime} \partial_{u_{1}^{\prime}}, \quad \partial_{v_{1}}=-u_{1}^{\prime} v_{1}^{\prime} \partial_{u_{1}^{\prime}}-v_{1}^{\prime 2} \partial_{v_{1}^{\prime}},
$$

and taking into account the local equations (B.1), we find that $\kappa$ is given by

$$
\begin{aligned}
\kappa\left(s_{1}^{\prime}, s_{1}\right)= & \left(\sum_{n \in \mathbb{Z}}\left(\alpha_{n}^{\prime}-\alpha_{-n}\right) t^{\prime n}\right) \partial_{x_{1}^{\prime}}+\left(\mu \sum_{n \in \mathbb{Z}}\left(\beta_{n}^{\prime}-\beta_{-n}\right) t^{\prime n}\right) \partial_{y_{1}^{\prime}} \\
& +\left(\sum_{n \in \mathbb{Z}}\left(\gamma_{n}^{\prime}-\gamma_{-n+d_{1}^{\prime}}\right) t^{\prime n}\right) \partial_{u_{1}^{\prime}}+\left(\sum_{n \in \mathbb{Z}}\left(\delta_{n}^{\prime}+\delta_{-n+2 d_{1}^{\prime}}\right) t^{\prime n}\right) \partial_{v_{1}^{\prime}} .
\end{aligned}
$$

Therefore, in order to find the kernel of $\kappa$ we have to solve the following system of equations

$$
\begin{aligned}
\alpha_{n}^{\prime}-\alpha_{-n} & =0, & & \beta_{n}^{\prime}-\beta_{-n}=0, \\
\gamma_{n}^{\prime}-\gamma_{-n+d_{1}^{\prime}} & =0, & & \delta_{n}^{\prime}+\delta_{-n+2 d_{1}^{\prime}}=0 .
\end{aligned}
$$

Since $\alpha_{n}^{\prime}, \ldots, \delta_{n}^{\prime}=0$ for $n<0$, we obtain

$$
\begin{array}{ll}
\alpha_{n}=0, & n>0, \quad \beta_{n}=0, \quad n>0, \\
\gamma_{n}=0, & n>d_{1}^{\prime}, \quad \delta_{n}=0, \quad n>2 d_{1}^{\prime} .
\end{array}
$$


Moreover, combining equations (B.6) and (B.10), we find the following relations

$$
\begin{aligned}
& \alpha_{n}=0, \quad n<-d_{1}^{\prime}, \quad \beta_{n}=0, \quad n<-2 d_{1}^{\prime}, \\
& \gamma_{n}=0, \quad n<0, \quad \delta_{n}=0, \quad n<0 .
\end{aligned}
$$

Therefore $\operatorname{Ker}(\kappa)=H^{0}(\Delta, \mathcal{T})$ is the $2\left(3 d_{1}^{\prime}+2\right)$-dimensional real vector space spanned by sections of the form

$$
\begin{aligned}
s_{1}= & \left(\sum_{n=-d_{1}^{\prime}}^{0} \alpha_{n} t^{n}\right) \partial_{x_{1}}+\left(\mu \sum_{n=-2 d_{1}^{\prime}}^{0} \beta_{n} t^{n}\right) \partial_{y_{1}} \\
& +\left(\sum_{n=0}^{d_{1}^{\prime}} \gamma_{n} t^{n}\right) \partial_{u_{1}}+\left(\sum_{n=0}^{2 d_{1}^{\prime}} \delta_{n} t^{n}\right) \partial_{v_{1}}
\end{aligned}
$$

with the coefficients $\alpha_{n}, \ldots, \delta_{n}$ subject to the boundary conditions (B.6). Next, we compute the cokernel of $\kappa$. This is generated by local sections with coefficients $a_{n}, \ldots, d_{n}$ for which the following equations have no solutions

$$
\begin{aligned}
\alpha_{n}^{\prime}-\alpha_{-n} & =a_{n}, & & \beta_{n}^{\prime}-\beta_{-n}=b_{n}, \\
\gamma_{n}^{\prime}-\gamma_{-n+d_{1}^{\prime}} & =c_{n}, & & \delta_{n}^{\prime}+\delta_{-n+2 d_{1}^{\prime}}=d_{n} .
\end{aligned}
$$

Taking into account equation (B.6) and the constraints $\alpha_{n}^{\prime}, \ldots, \gamma_{n}^{\prime}=0$, it is straightforward to check that these equations admit nontrivial solutions for any values of $a_{n}, \ldots, d_{n}$. Therefore, $\operatorname{Coker}(\kappa)=H^{1}\left(\Delta, \mathcal{T}_{\Delta}\right)$ is trivial.

In conclusion, as shown in Section 6.3, the space of infinitesimal deformations of the multicover (B.3) is isomorphic to $H^{0}(\Delta, \mathcal{T})$. Although this is a priori only a real vector space, it should carry a complex structure reflecting the choice of an orientation on the moduli space of open string maps. This is a very subtle issue since we do not have a rigorous construction of the moduli space and the virtual fundamental class. Here we will simply choose the complex structure defined by the isomorphism $H^{0}(\Delta, \mathcal{T}) \longrightarrow \mathbb{C}^{3 d_{1}^{\prime}+1}, s_{1} \longrightarrow\left(\alpha_{m}, \beta_{n}\right), m=1, \ldots, d_{1}^{\prime}, n=1, \ldots, 2 d_{1}^{\prime}$. With this choice, $H^{0}\left(\Delta, \mathcal{T}_{\Delta}\right)$ is $S^{1}$-isomorphic to

$$
\bigoplus_{n=0}^{d_{1}^{\prime}}\left(-\lambda_{1}+\frac{n}{d_{1}^{\prime}} \lambda_{3}\right) \oplus \bigoplus_{n=0}^{2 d_{1}^{\prime}}\left(-\lambda_{3}+\frac{n}{d_{1}^{\prime}} \lambda_{3}\right) \text {. }
$$

One could in principle make other choices by choosing different isomoprhisms to $\mathbb{C}^{3 d_{1}^{\prime}+2}$, e.g., $s_{1} \longrightarrow\left(\gamma_{m}, \delta_{n}\right), m=1, \ldots, d_{1}^{\prime}, n=1, \ldots, 2 d_{1}^{\prime}$. This would change the representations in equation(B.14) by complex conjugation, and it would reflect in a different sign of the multicover contribution to the instanton sum. 
Now we turn to the computation of $H^{0}\left(\Delta, \mathcal{L}_{\Delta}\right)$. We construct the Čech complex

$$
0 \longrightarrow \mathcal{L}_{\Delta}\left(\Upsilon_{1}^{\prime}\right) \oplus \mathcal{L}_{\Delta}\left(\Upsilon_{1}\right) \stackrel{\kappa}{\longrightarrow} \mathcal{L}_{\Delta}\left(\Upsilon_{1}^{\prime} \cap \Upsilon_{1}\right) \longrightarrow 0
$$

The generic sections of $\mathcal{L}$ over $\Upsilon_{1}^{\prime}$ and $\Upsilon_{1}$ have the form

$$
\begin{aligned}
& \tilde{s}_{1}^{\prime}=\left(\sum_{n=0}^{\infty} \epsilon_{n}^{\prime} t^{\prime n}\right) \Lambda^{\prime \max }, \\
& \tilde{s}_{1}=\left(\sum_{n \in \mathbb{Z}} \epsilon_{n} t^{n}\right) \Lambda^{\max },
\end{aligned}
$$

where $\quad \Lambda^{\prime \max }=\partial_{x_{1}^{\prime}} \wedge \partial_{y_{1}^{\prime}} \wedge \partial_{u_{1}^{\prime}} \wedge \partial_{v_{1}^{\prime}} \quad$ and $\quad \Lambda^{\max }=\partial_{x_{1}} \wedge \partial_{y_{1}} \wedge \partial_{u_{1}} \wedge \partial_{v_{1}}$. Using equation (B.7), we find that $\kappa$ is given by

$$
\kappa\left(\tilde{s}_{1}^{\prime}, \tilde{s}_{1}\right)=\left(\sum_{n \in \mathbb{Z}}\left(\epsilon_{n}^{\prime}-\epsilon_{-n+3 d_{1}^{\prime}}\right) t^{\prime n}\right) \Lambda^{\prime \max },
$$

where it is understood that $\epsilon_{n}^{\prime}=0$ for $n<0$. Then, after using the boundary condition $\epsilon_{n}=\bar{\epsilon}_{-n}$, we find that the kernel of $\kappa$ is generated by sections of the form

$$
\tilde{s}_{1}=\left(\epsilon_{0}+\sum_{n=1}^{3 d_{1}^{\prime}}\left(\epsilon_{n} t^{n}+\bar{\epsilon}_{n} t^{-n}\right)\right) \Lambda^{\max } .
$$

This is a $6 d_{1}^{\prime}+1$ real vector space which splits naturally in a direct sum of the form $\mathbb{R} \oplus \mathbb{R}^{6 d_{1}^{\prime}}$. The first summand is generated by sections of the form $\epsilon_{0} \Lambda^{\max }$, and the second summand is defined by sections $\tilde{s}_{1}$ with $\epsilon_{0}=0$. One can define a complex structure on the subspace $\epsilon_{0}=0$ by an isomor-

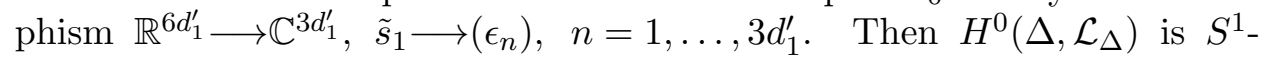
isomorphic to

$$
(0)_{\mathbb{R}} \oplus \bigoplus_{n=1}^{3 d_{1}^{\prime}}\left(\frac{n \lambda_{3}}{d_{1}^{\prime}}\right) .
$$

Analogous considerations show that the cokernel of $\kappa$ is empty in this case, and therefore $H^{1}\left(\Delta, \mathcal{L}_{\Delta}\right)=0$.

\section{B.2 Multicovers of $D_{3}^{\prime}$}

Since $D_{1}^{\prime}$ and $D_{3}^{\prime}$ are complementary discs in $C_{13}^{\prime}, D_{3}^{\prime}$ is covered by the coordinate patches $\mathcal{U}_{1}=\left\{Z_{2} \neq 0, Z_{4} \neq 0, W \neq 0\right\}, \mathcal{U}_{3}=\left\{Z_{2} \neq 0, Z_{3} \neq 0, W \neq 0\right\}$. 
The local coordinates in the second patch are

$$
\mathcal{U}_{3}: \quad x_{3}=\frac{Z_{1}}{Z_{2}}, \quad y_{3}=\frac{Z_{4}}{Z_{3}}, \quad u_{3}=\frac{U Z_{2} Z_{3}}{W}, \quad v_{3}=\frac{V Z_{2} Z_{3}}{W}
$$

and $D_{3}^{\prime}$ is given by

$$
\begin{aligned}
& \mathcal{U}_{1}: \quad x_{1}=u_{1}=0, \quad y_{1} v_{1}=\mu, \quad\left|y_{1}\right| \geq \mu^{1 / 2} \quad\left|v_{1}\right| \leq \mu^{1 / 2} . \\
& \mathcal{U}_{3}: \quad x_{3}=u_{3}=0, \quad v_{3}=\mu, \quad\left|y_{3}\right| \leq \mu^{-1 / 2}
\end{aligned}
$$

We choose an open cover of the domain of the form

$$
\Upsilon_{1}=\left\{\mu^{1 / 2 d_{3}^{\prime}} \leq|t|<\left(\mu+\epsilon^{2}\right)^{1 / 2 d_{3}^{\prime}}\right\}, \quad \Upsilon_{3}=\left\{0 \leq\left|t^{\prime}\right|<\mu^{-1 / 2 d_{3}^{\prime}}\right\}
$$

A Galois cover of degree $d_{3}^{\prime}$ of $D_{3}^{\prime}$ is given in local coordinates by

$$
\begin{aligned}
& \mathcal{U}_{1}: \quad x_{1}(t)=0, \quad y_{1}(t)=t^{d_{3}^{\prime}}, \quad u_{1}(t)=0, \quad v_{1}=\mu t^{-d_{3}^{\prime}} \\
& \mathcal{U}_{3}: \quad x_{3}\left(t^{\prime}\right)=0, \quad y_{3}\left(t^{\prime}\right)=t^{\prime d_{3}^{\prime}}, \quad u_{3}\left(t^{\prime}\right)=0, \quad v_{3}\left(t^{\prime}\right)=\mu
\end{aligned}
$$

The Čech complex for $\mathcal{T}$ is

$$
0 \longrightarrow \mathcal{T}_{\Delta}\left(\Upsilon_{1}\right) \oplus \mathcal{T}_{\Delta}\left(\Upsilon_{3}\right) \stackrel{\kappa}{\longrightarrow} \mathcal{T}_{\Delta}\left(\Upsilon_{1} \cap \Upsilon_{3}\right) \longrightarrow 0
$$

with generic sections in $\mathcal{T}_{\Delta}\left(\Upsilon_{1}\right)$ and $\mathcal{T}_{\Delta}\left(\Upsilon_{3}\right)$ given by

$$
\begin{aligned}
s_{1}= & \left(\sum_{n \in \mathbb{Z}} \alpha_{n} t^{n}\right) \partial_{x_{1}}+\left(\sum_{n \in \mathbb{Z}} \beta_{n} t^{n}\right) \partial_{y_{1}}+\left(\sum_{n \in \mathbb{Z}} \gamma_{n} t^{n}\right) \partial_{u_{1}} \\
& +\left(\mu \sum_{n \in \mathbb{Z}} \delta_{n} t^{n}\right) \partial_{v_{1}} \\
s_{3}= & \left(\sum_{n=0}^{\infty} \alpha_{n}^{\prime} t^{\prime n}\right) \partial_{x_{3}}+\left(\sum_{n=0}^{\infty} \beta_{n}^{\prime} t^{\prime n}\right) \partial_{y_{3}}+\left(\sum_{n=0}^{\infty} \gamma_{n}^{\prime} t^{\prime n}\right) \partial_{u_{3}} \\
& +\left(\mu \sum_{n=0}^{\infty} \delta_{n}^{\prime} t^{\prime n}\right) \partial_{v_{3}}
\end{aligned}
$$

Note that we sum over $n \geq 0$ for sections in $\mathcal{T}_{\Delta}\left(\Upsilon_{3}\right)$. In order to have a uniform notation, we can extend these sums to $n \in \mathbb{Z}$, with the convention 
that $\alpha_{n}^{\prime}, \ldots, \delta_{n}^{\prime}$ are zero for $n<0$. Using the linear transformations $\partial_{x_{1}}=\partial_{x_{3}}, \quad \partial_{y_{1}}=-y_{3}^{2} \partial_{y_{3}}+y_{3} v_{3} \partial_{v_{3}}, \quad \partial_{u_{1}}=y_{3}^{-1} \partial_{u_{3}}, \quad \partial_{v_{1}}=y_{3}^{-1} \partial_{v_{3}}$,

and proceeding as in the previous case, we obtain the following form for the Čech differential

$$
\begin{aligned}
\kappa\left(s_{1}, s_{3}\right)= & \left(\sum_{n \in \mathbb{Z}}\left(\alpha_{n}^{\prime}-\alpha_{-n}\right) t^{\prime n}\right) \partial_{x_{3}}+\left(\sum_{n \in \mathbb{Z}}\left(\beta_{n}^{\prime}+\beta_{-n+2 d_{3}^{\prime}}\right) t^{\prime n}\right) \partial_{y_{3}} \\
& +\left(\sum_{n \in \mathbb{Z}}\left(\gamma_{n}^{\prime}-\gamma_{-n-d_{3}^{\prime}}\right) t^{\prime n}\right) \partial_{u_{3}} \\
& +\left(\mu \sum_{n \in \mathbb{Z}}\left(\delta_{n}^{\prime}-\beta_{-n+d_{3}^{\prime}}-\delta_{-n-d_{3}^{\prime}}\right) t^{\prime n}\right) \partial_{v_{3}} .
\end{aligned}
$$

Again, in order to determine $H^{0}\left(\Delta, \mathcal{T}_{\Delta}\right)$, we have to compute the kernel of $\kappa$, which is determined by the following system of equations

$$
\begin{aligned}
& \alpha_{n}^{\prime}=\alpha_{-n}, \quad \beta_{n}^{\prime}=-\beta_{-n+2 d_{3}^{\prime}}, \quad \gamma_{n}^{\prime}=\gamma_{-n-d_{3}^{\prime}}, \\
& \delta_{n}^{\prime}=\beta_{-n+d_{3}^{\prime}}+\delta_{-n-d_{3}^{\prime}} \text {. }
\end{aligned}
$$

But $\alpha_{n}^{\prime}, \ldots, \delta_{n}^{\prime}=0$ for $n<0$, and we obtain that

$$
\begin{gathered}
\alpha_{n}=0, \quad n>0, \quad \beta_{n}=0, \quad n>2 d_{3}^{\prime}, \quad \gamma_{n}=0, \quad n>-d_{3}^{\prime}, \\
\beta_{-n+d_{3}^{\prime}}+\delta_{-n-d_{3}^{\prime}}=0, \quad n<0 .
\end{gathered}
$$

We will also need to employ boundary conditions at $|t|=\mu^{1 / 2 d_{3}^{\prime}}$ which yield the following relations

$$
\alpha_{n}=\mu^{-n / d_{3}^{\prime}} \bar{\gamma}_{-n}, \quad \beta_{n}=\mu^{\left(d_{3}^{\prime}-n\right) / d_{3}^{\prime}} \bar{\delta}_{-n} .
$$

Using equations (B.29) and (B.30), we obtain

$$
\begin{gathered}
\alpha_{n}=0, \quad n<d_{3}^{\prime}, \quad \beta_{n}=0, \quad n<0, \quad \gamma_{n}=0, \quad n<0, \\
\delta_{n}=0, \quad n<-2 d_{3}^{\prime} \quad \text { or } \quad n>0, \\
\beta_{d_{3}^{\prime}+1}=\bar{\beta}_{d_{3}^{\prime}-1}, \quad \delta_{-d_{3}^{\prime}+1}=\bar{\delta}_{-d_{3}^{\prime}-1} .
\end{gathered}
$$

Taking into account all constraints, we find that the kernel of $\kappa$ is generated by sections of the form

$$
\begin{aligned}
s_{1}= & {\left[\sum_{n=0}^{d_{3}^{\prime}-1}\left(\beta_{n} t^{n}-\bar{\beta}_{n} t^{n+d_{3}^{\prime}+1}\right)+\beta_{d_{3}^{\prime}} t^{d_{3}^{\prime}}\right] \partial_{y_{1}} } \\
& +\left[\mu \sum_{n=-d_{3}^{\prime}+1}^{0}\left(\delta_{n} t^{n}-\bar{\delta}_{n} t^{-n-d_{3}^{\prime}-1}\right)+\delta_{-d_{3}^{\prime}} t^{-d_{3}^{\prime}}\right] \partial_{v_{1}},
\end{aligned}
$$


with coefficients $\beta_{n}$ and $\delta_{n}$ subject to the boundary conditions (B.30). Choosing $\delta_{n}, n=-d_{3}^{\prime}, \ldots, 0$, to be holomorphic coordinates, the $S^{1}$-equivariant decomposition of $H^{0}\left(\Delta, \mathcal{T}_{\Delta}\right)$ reads

$$
\bigoplus_{n=0}^{d_{3}^{\prime}}\left(-\lambda_{3}+\frac{n}{d_{3}^{\prime}} \lambda_{3}\right)
$$

Next, proceeding as in the previous case, we find that the cokernel of $\kappa$ is generated by sections of the form

$$
s_{1}=\left(\sum_{n=1}^{d_{3}^{\prime}-1} \alpha_{n} t^{n}\right) \partial_{x_{1}}+\left(\sum_{n=-d_{3}^{\prime}+1}^{-1} \gamma_{n} t^{n}\right) \partial_{u_{1}},
$$

with coefficients $\alpha_{n}$ and $\beta_{n}$ subject to the boundary conditions (B.30). If we choose $\gamma_{n}, n=-d_{3}^{\prime}+1, \ldots,-1$, to be holomorphic coordinates, $H^{1}\left(\Delta, \mathcal{T}_{\Delta}\right)$ is $S^{1}$-isomorphic to

$$
\bigoplus_{n=1}^{d_{3}^{\prime}-1}\left(\lambda_{1}-\frac{n}{d_{3}^{\prime}} \lambda_{3}\right)
$$

Finally, we are left with $H^{0}\left(\Delta, \mathcal{L}_{\Delta}\right)$. In this case, the Čech complex reads

$$
0 \longrightarrow \mathcal{L}_{\Delta}\left(\Upsilon_{1}\right) \oplus \mathcal{L}_{\Delta}\left(\Upsilon_{3}\right) \stackrel{\kappa}{\longrightarrow} \mathcal{L}_{\Delta}\left(\Upsilon_{1} \cap \Upsilon_{3}\right) \longrightarrow 0
$$

The generic sections of $\mathcal{L}$ over $\Upsilon_{1}$ and $\Upsilon_{3}$ have the form

$$
\begin{aligned}
& \tilde{s}_{1}=\left(\sum_{n \in \mathbb{Z}} \epsilon_{n} t^{n}\right) \Lambda_{1}^{\max }, \\
& \tilde{s}_{3}=\left(\sum_{n=0}^{\infty} \epsilon_{n}^{\prime} t^{\prime n}\right) \Lambda_{3}^{\max },
\end{aligned}
$$

where $\Lambda_{1}^{\max }=\partial_{x_{1}} \wedge \partial_{y_{1}} \wedge \partial_{u_{1}} \wedge \partial_{v_{1}}$ and $\Lambda_{3}^{\max }=\partial_{x_{3}} \wedge \partial_{y_{3}} \wedge \partial_{u_{3}} \wedge \partial_{v_{3}}$. Using equation (B.26), we find that $\kappa$ is given by

$$
\kappa\left(\tilde{s}_{1}, \tilde{s}_{3}\right)=\left(\sum_{n \in \mathbb{Z}}\left(\epsilon_{n}^{\prime}-\epsilon_{-n}\right) t^{\prime n}\right) \Lambda_{3}^{\max },
$$

where it is understood that $\epsilon_{n}^{\prime}=0$ for $n<0$. Then, also using the boundary condition $\epsilon_{n}=\bar{\epsilon}_{-n}$, it follows that the kernel of $\kappa$ is generated by sections of the form

$$
\tilde{s}_{1}=\epsilon_{0} \Lambda_{1}^{\max } .
$$

Therefore, $H^{0}\left(\Delta, \mathcal{L}_{\Delta}\right)$ is $S^{1}$-isomorphic to $(0)_{\mathbb{R}}$. A similar analysis shows that $H^{1}\left(\Delta, \mathcal{L}_{\Delta}\right)$ is trivial. 
To conclude this section, note that horizontal discs such as $D_{3}^{\prime}$ are embedded in the smooth component $\bar{Y}_{1}$ of $\bar{Y}$, and have no common points with the singular divisor. This is already clear from the local analysis in Section 5 . Then one can compute the same multicover contributions by taking open string maps to $\bar{Y}_{1}$ as in ref. [16] instead of $\mathcal{Z}$. If the formalism proposed here is robust, this point of view should give the same results as above. As this computation has been performed in detail in ref. [16], let us just record the results. With suitable choices of complex structures, we have

$$
\begin{aligned}
& H^{0}(\Delta, \mathcal{T})_{\bar{Y}} \simeq(0)_{\mathbb{R}} \oplus \bigoplus_{n=0}^{d_{3}^{\prime}-1}\left(-\lambda_{3}+\frac{n}{d_{3}^{\prime}} \lambda_{3}\right), \\
& H^{1}(\Delta, \mathcal{T})_{\bar{Y}} \simeq \bigoplus_{n=1}^{d_{3}^{\prime}-1}\left(\lambda_{1}-\frac{n}{d_{3}^{\prime}} \lambda_{3}\right) .
\end{aligned}
$$

Note that in this case there is no obstruction bundle since $D_{3}^{\prime}$ is a rigid disc on $\bar{Y}_{1}$ supported away from the canonical class. Therefore, the expected dimension of the moduli space is zero [33]. The formulae (B.41) are very similar to equations (B.34) and (B.36) except for a small discrepancy in the fixed parts of the deformation spaces $H^{0}(\Delta, \mathcal{T})$ and $H^{0}(\Delta, \mathcal{T})_{\bar{Y}}$. For maps to $\mathcal{Z}$, one finds that the fixed part is $(0)_{\mathbb{C}}$ corresponding to the term $n=d_{3}^{\prime}$ in equation (B.34), while for maps to $\bar{Y}$ the fixed part is $(0)_{\mathbb{R}}$. This discrepancy is accounted for by the fixed part of the obstruction space, as explained in Section 6.3. The final result is that the two contributions agree, as expected.

\section{Appendix C Chern-Simons expectation values}

The Chern-Simons expansion performed in Section 7 involves products of holonomy variables in $U(N)$ representations of the form $R_{i}$ and $\bar{R}_{j}$, where $R_{i}$ and $R_{j}$ are defined by Young tableaux. Such expressions can usually be avoided in the context of large $N$ duality literature [38, 40,47, 52, 54] by analytic continuation. This is no longer true in compact examples, and hence we have to evaluate such knot and link invariants by direct methods. We only need to consider two cases.

(i) Consider unknot invariants of the form

$$
\left\langle\operatorname{Tr}_{R_{1}} V \operatorname{Tr}_{\bar{R}_{2}} V\right\rangle_{p}
$$


where $p$ denotes the framing. Proceeding by analogy with refs. $[38,40$, $47,52,54]$, we write

$$
\left\langle\operatorname{Tr}_{R_{1}} V \operatorname{Tr}_{\bar{R}_{2}} V\right\rangle_{p}=\sum_{\rho}\left\langle\operatorname{Tr}_{\rho} V\right\rangle_{p}
$$

where

$$
R_{1} \otimes \bar{R}_{2}=\oplus_{\rho} \rho
$$

is the irreducible decomposition of the product representation. Each term in the right-hand side of equation (C.2) can then be evaluated according to the rules explained in ref. [47]

$$
\left\langle\operatorname{Tr}_{\rho} V\right\rangle_{p}=x^{p C \rho}\left\langle\operatorname{Tr}_{\rho} V\right\rangle_{0}
$$

where $x=\mathrm{e}^{\mathrm{i} \pi /(k+N)}$ and $C_{\rho}$ is the second Casimir of $\rho$. Therefore, the computation reduces to some standard representation theory and expectation values of the unknot in the canonical framing. Given a $\mathrm{SU}(N)$ representation $R$ defined by a Young tableau $\Pi, \bar{R}$ is isomorphic to the $\mathrm{SU}(N)$ representation defined by the complementary Young tableau $\bar{\Pi}$. Two Young tableaux $\Pi$ and $\bar{\Pi}$ are called complementary if by adjoining the $(N-k)$ th row of $\bar{\Pi}$ to the $k$ th row of $\Pi$ we obtain a square tableau with $N \times N$ boxes. Then, for two $\mathrm{SU}(N)$ representations $R_{1}$ and $\bar{R}_{2}$, one can compute the irreducible decomposition (C.3) using the standard rules for Young tableaux. The irreducible decomposition for $U(N)$ representations can be obtained by tensoring the $\mathrm{SU}(N)$ decomposition by appropriate $U(1)$ representations. Using these rules, we find the following relations

$$
\begin{aligned}
\square \otimes \bar{\square} & =\mathbb{1} \oplus A_{1}, \\
\square \otimes \bar{\square} & =\square \oplus A_{2}, \\
\boxminus \otimes \bar{\square} & =\square \oplus A_{3},
\end{aligned}
$$

where $A_{1}, A_{2}$ and $A_{3}$ are irreducible representations of $U(N)$ given by

$$
A_{1}=\underset{\square}{\vdots} \otimes(-\sqrt{N}), \quad A_{2}=\underset{\square}{\square} \otimes(-\sqrt{N}), \quad A_{3}=\underset{\square}{\square} \otimes(-\sqrt{N}) .
$$

The number of boxes in the first column of each tableau in equation (C.6) is $N-1$ and $(w)$ denotes a one-dimensional $U(1)$ representation of charge $w$. A routine computation shows that the dimensions and the quadratic 
Casimir operators of $A_{1}, A_{2}$ and $A_{3}$ are

$$
\begin{aligned}
\operatorname{dim}\left(A_{1}\right) & =N^{2}-1, \quad \operatorname{dim}\left(A_{2}\right)=\frac{N(N-1)(N+2)}{2} \\
\operatorname{dim}\left(A_{3}\right) & =\frac{N(N+1)(N-2)}{2}, \\
C_{2}\left(A_{1}\right) & =2 N, \quad C_{2}\left(A_{2}\right)=3 N+2, \quad C_{2}\left(A_{3}\right)=3 N-2 .
\end{aligned}
$$

Furthermore, we have the following relations

$$
\square \otimes A_{1}=\square \oplus A_{2} \oplus A_{3}, \quad \bar{\square} \otimes \bar{A}_{1}=\bar{\square} \oplus \bar{A}_{2} \oplus \bar{A}_{3} .
$$

The unknot expectation values in canonical framing can be computed using the rules explained in refs. [47,52]. The $U(1)$ factor decouples, and hence $U(N)$ expectation values are the same as $\mathrm{SU}(N)$ expectation values. The later are of the form $\left\langle\operatorname{Tr}_{R} V\right\rangle=\operatorname{Tr}_{R} U_{0}$, where $U_{0}$ is a fixed group element. Using this property, one can easily show that

$$
\begin{aligned}
& \left\langle\operatorname{Tr}_{A_{1}} V\right\rangle_{0}=\left(\left\langle\operatorname{Tr}_{\square} V\right\rangle_{0}\right)^{2}-1, \\
& \left\langle\operatorname{Tr}_{A_{2}} V\right\rangle_{0}=\left\langle\operatorname{Tr}_{\square} V\right\rangle_{0}\left(\left\langle\operatorname{Tr}_{\square} V\right\rangle_{0}-1\right), \\
& \left\langle\operatorname{Tr}_{A_{3}} V\right\rangle_{0}=\left\langle\operatorname{Tr}_{\square} V\right\rangle_{0}\left(\left\langle\operatorname{Tr}_{\square} V\right\rangle_{0}-1\right) .
\end{aligned}
$$

Using equations (C.4) and (C.9) and using the normalization explained in Section 5, we find

$$
\begin{aligned}
\left\langle\operatorname{Tr}_{\square} V \operatorname{Tr}_{\square} V\right\rangle & =y^{-2}+\left\langle\operatorname{Tr}_{\square} V\right\rangle_{0}^{2}-1, \\
\left\langle\operatorname{Tr}_{\square} V \operatorname{Tr}_{\bar{\square}} V\right\rangle & =\left\langle\operatorname{Tr}_{\bar{\square}} V \operatorname{Tr}_{\square} V\right\rangle \\
& =y^{-2}\left\langle\operatorname{Tr}_{\square} V\right\rangle_{0}+x^{2 p}\left\langle\operatorname{Tr}_{\square} V\right\rangle_{0}\left(\left\langle\operatorname{Tr}_{\square} V\right\rangle_{0}-1\right), \\
\left\langle\operatorname{Tr}_{\boxminus} V \operatorname{Tr}_{\square} V\right\rangle & =\left\langle\operatorname{Tr}_{\bar{G}} V \operatorname{Tr}_{\square} V\right\rangle \\
& =y^{-2}\left\langle\operatorname{Tr}_{\square} V\right\rangle_{0}+x^{-2 p}\left\langle\operatorname{Tr}_{\square} V\right\rangle_{0}\left(\left\langle\operatorname{Tr}_{\boxminus} V\right\rangle_{0}-1\right) .
\end{aligned}
$$

(ii) We also encounter expectation values of the form

$$
\left\langle\operatorname{Tr}_{R_{1}} V \operatorname{Tr}_{R_{2}} U\right\rangle_{p_{1}, p_{2}}^{+1}
$$

for a Hopf link with linking number +1 and framings $\left(p_{1}, p_{2}\right)$. This computation can be reduced to a Hopf link with $l=-1$ and canonical 
framing on both components as follows

$$
\left\langle\operatorname{Tr}_{R_{1}} V \operatorname{Tr}_{R_{2}} U\right\rangle_{p_{1}, p_{2}}^{+1}=x^{p_{1} C_{R_{1}}+p_{2} C_{R_{2}}}\left\langle\operatorname{Tr}_{R_{1}} V \operatorname{Tr}_{R_{2}} U\right\rangle_{0,0}^{-1}\left(x \rightarrow x^{-1}\right) .
$$

The correctly normalized link invariant in the context of geometric transitions [38]-[40] reads

$$
\left\langle\operatorname{Tr}_{\square} V \operatorname{Tr}_{\bar{\square}} U\right\rangle_{0,0}^{-1}=x^{\left(C_{2}(\square)+C_{2}(\bar{\square})\right)} \sum_{\rho \in \square \otimes \bar{\square}} x^{-C_{2}(\rho)} \operatorname{dim}_{q} \rho .
$$

Dropping again the trivial framing factors, we finally obtain

$$
\begin{aligned}
\left\langle\operatorname{Tr}_{\bar{\square}} V \operatorname{Tr}_{\bar{\square}} U\right\rangle= & \left\langle\operatorname{Tr}_{\bar{\square}} V \operatorname{Tr}_{\square} U\right\rangle=y^{-2}+\left\langle\operatorname{Tr}_{\square} V\right\rangle_{0}^{2}-1, \\
\left\langle\operatorname{Tr}_{\square} V \operatorname{Tr}_{\bar{\square}} V\right\rangle= & \left\langle\operatorname{Tr}_{\bar{\square}} V \operatorname{Tr}_{\square} V\right\rangle \\
= & y^{-2} x^{2(p-1)}\langle\operatorname{Tr} V\rangle_{0}+x^{2 p}\langle\operatorname{Tr} V\rangle_{0}\left(\left\langle\operatorname{Tr}_{\square} V\right\rangle_{0}-1\right), \\
\left\langle\operatorname{Tr}_{\boxminus} V \operatorname{Tr}_{\bar{\square}} V\right\rangle= & \left\langle\operatorname{Tr}_{\bar{\Xi}} V \operatorname{Tr}_{\square} V\right\rangle \\
= & y^{-2} x^{-2(p-1)}\langle\operatorname{Tr} V\rangle_{0}+x^{-2 p}\langle\operatorname{Tr} V\rangle_{0}\left(\left\langle\operatorname{Tr}_{\boxminus} V\right\rangle_{0}-1\right), \\
\left\langle\operatorname{Tr} V \operatorname{Tr}_{\bar{\square}} V \operatorname{Tr} U\right\rangle= & \left\langle\operatorname{Tr} V \operatorname{Tr}_{\bar{\square}} V \operatorname{Tr}_{\bar{\square}} U\right\rangle \\
= & 2 y^{-2}\langle\operatorname{Tr} V\rangle_{0}+x^{2}\langle\operatorname{Tr} V\rangle_{0}\left(\left\langle\operatorname{Tr}_{\square} V\right\rangle_{0}-1\right) \\
& +x^{-2}\left(\left\langle\operatorname{Tr}_{\boxminus} V\right\rangle_{0}-1\right) .
\end{aligned}
$$

\section{Acknowledgments}

This work is a spin-off of a broader research program initiated by Ron Donagi, Antonella Grassi, Tony Pantev and us. It is a pleasure to thank them for collaboration and sharing their ideas with us during the completion of this project. D.-E.D. would like to thank Mina Aganagic, Marcos Mariño and Cumrun Vafa for collaboration on a related project and valuable discussions. B.F. would like to thank Philip Candelas and Harald Skarke for helpful conversations. This work was partially supported by DOE grant DOE-DE-FG02-96ER40959.

\section{References}

[1] M. Aganagic and C. Vafa, Mirror symmetry, D-branes and counting holomorphic discs, hep-th/0012041.

[2] M. Aganagic, A. Klemm and C. Vafa, Disk instantons, mirror symmetry and the duality web, Z. Naturforsch. A57 (2002), 1, hep-th/0105045. 
[3] M. Aganagic, A. Klemm, M. Mariño and C. Vafa, Matrix model as a mirror of Chern-Simons theory, hep-th/0211098.

[4] M. Aganagic and C. Vafa, $G_{2}$ manifolds, mirror symmetry and geometric engineering, hep-th/0110171.

[5] M. Aganagic, M. Mariño and C. Vafa, All loop topological string amplitudes from Chern-Simons theory, hep-th/0206164.

[6] A.C. Avram, P. Candelas, D. Jančić and M. Mandelberg, On the connectedness of the moduli space of Calabi-Yau manifolds, Nucl. Phys. B 465 (1996), 458, hep-th/9511230.

[7] V. Batyrev, Dual polyhedra and mirror symmetry for Calabi-Yau hypersurfaces in toric varieties, J. Algebr. Geom. 3 (1994), 493.

[8] P. Berglund, S. Katz and A. Klemm, Mirror symmetry and the moduli space for generic hypersurfaces in toric varieties, Nucl. Phys. B 456 (1995), 153, hep-th/9506091.

[9] K. Behrend, Gromov-Witten invariants in algebraic geometry, Invent. Math. 127 (1997), 601.

[10] K. Behrend and B. Fantechi, The intrinsic normal cone, Invent. Math. 128 (1997), 45.

[11] P. Candelas, X. de la Ossa, A. Font, S. Katz and D.R. Morrison, Mirror symmetry for two parameter models I, Nucl. Phys. B 416 (1994), 481, hep-th/9308083.

[12] D.A. Cox and S. Katz, Mirror symmetry and algebraic geometry, Mathematical Surveys and Monographs, 68, Providence, 1999.

[13] T.-M. Chiang, A. Klemm, S.-T. Yau and E. Zaslow, Local mirror symmetry: calculations and interpretations, ATMP 3 (1999), 495, hep-th/9903053.

[14] H. Clemens, Double solids, Adv. Math. 47 (1983), 107.

[15] D.-E. Diaconescu, B. Florea and A. Grassi, Geometric transitions and open string instantons, ATMP 6 (2002), hep-th/0205234.

[16] D.-E. Diaconescu, B. Florea and A. Grassi, Geometric transitions, del Pezzo surfaces and open string instantons, ATMP 6 (2002), hep-th/0206163.

[17] R. Dijkgraaf and C. Vafa, Matrix models, topological strings, and supersymmetric gauge theories, Nucl. Phys. B 644 (2002), 3, hep-th/ 0206255.

[18] R. Dijkgraaf and C. Vafa, On geometry and matrix models, Nucl. Phys. B 644 (2002) 21, hep-th/0207106.

[19] C. Faber and R. Pandharipande, Hodge integrals and Gromov-Witten theory, Invent. Math. 139 (2000), 139, math.AG/9810173. 
[20] T. Graber and R. Pandharipande, Localization of virtual classes, Invent. Math. 135 (1999), 487, math.AG/9708001.

[21] R. Gopakumar and C. Vafa, Topological gravity as large $N$ topological gauge theory, ATMP 2 (1998), 413, hep-th/9802016.

[22] R. Gopakumar and C. Vafa, M-theory and topological strings I, hepth/9809187; M-theory and topological strings II, hep-th/9812127.

[23] R. Gopakumar and C. Vafa, On the gauge theory/geometry correspondence, ATMP 3 (1999), 1415, hep-th/9811131.

[24] S. Govindarajan, T. Jayaraman and T. Sarkar, Disc instantons in linear sigma models, hep-th/0108234.

[25] T. Graber and E. Zaslow, Open string Gromov-Witten invariants: calculations and a mirror 'theorem', hep-th/0109075.

[26] B.R. Greene, D.R. Morrison and A. Strominger, Black hole condensation and the unification of string vacua, Nucl. Phys. B 451 (1995), 109, hep-th/9504145.

[27] S. Hosono, A. Klemm, S. Theisen and S.-T. Yau, Mirror symmetry, mirror map and applications to Calabi-Yau hypersurfaces, Commun. Math. Phys. 167 (1995), 301, hep-th/9308122.

[28] S. Hosono, B.H. Lian and S.-T. Yau, GKZ-generalized hypergeometric systems in mirror symmetry of Calabi-Yau hypersurfaces, Commun. Math. Phys. 182 (1996), 535, hep-th/9511001.

[29] A. Iqbal, All genus topological string amplitudes and 5-brane webs as Feynman diagrams, hep-th/0207114.

[30] A. Iqbal and A.-K. Kashani-Poor, Instanton counting and ChernSimons theory, hep-th/0212279.

[31] S. Kachru, S. Katz, A. Lawrence and J. McGreevy, Open string instantons and superpotentials, Phys. Rev. D 62 (2000), 026001, hep-th/9912151.

[32] S. Kachru, S. Katz, A. Lawrence and J. McGreevy, Mirror symmetry for open strings, hep-th/0006047.

[33] S. Katz and C.-C.M. Liu, Enumerative geometry of stable maps with Lagrangian boundary conditions and multiple covers of the disc, ATMP 5 (2001), 1, math.AG/0103074.

[34] S. Katz and S.A. Strømme, SCHUBERT, a maple package for intersection theory and enumerative geometry, http://www.mi.uib.no/ schubert.

[35] A. Klemm, S. Katz and C. Vafa, M-theory, topological strings and spinning black holes, ATMP 3 (1999), 1445, hep-th/9910181. 
[36] M. Kontsevich, Enumeration of rational curves via torus actions, in 'The Moduli Space of Curves', Progr. Math., 129, Birkhäuser, Boston, MA, 1995, 335-368.

[37] M. Kreuzer and H. Skarke, PALP: a package for analyzing lattice polytopes with applications to toric geometry, http://hep.

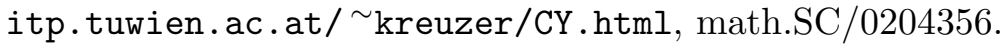

[38] J.M.F. Labastida and M. Mariño, Polynomial invariants for torus knots and topological strings, Commun. Math. Phys. 217 (2001), 423, hep-th/0004196.

[39] J.M.F. Labastida, M. Mariño, and C. Vafa, Knots, links and branes at large $N$, JHEP 11 (2000), hep-th/0010102.

[40] J.M.F. Labastida and M. Mariño, A new point of view in the theory of knot and link invariants, math.QA/0104180.

[41] W. Lerche and P. Mayr, On $\mathcal{N}=1$ mirror symmetry for open type II strings, hep-th/0111113.

[42] W. Lerche, P. Mayr and N. Warner, Holomorphic $\mathcal{N}=1$ special geometry of open-closed type II strings, hep-th/0207259.

[43] W. Lerche, P. Mayr and N. Warner, $\mathcal{N}=1$ special geometry, mixed Hodge variations and toric geometry, hep-th/0208039.

[44] J. Li and G. Tian, Virtual moduli cycles and Gromov-Witten invariants of algebraic varieties, J. Am. Math. Soc. 11 (1998), 119.

[45] J. Li and Y.S. Song, Open string instantons and relative stable morphisms, hep-th/0103100.

[46] Y.I. Manin, Frobenius Manifolds, Quantum Cohomology, and Moduli Spaces, American Mathematical Society Colloquium Publications, 47, 1999.

[47] M. Mariño and C. Vafa, Framed knots at large N, hep-th/0108064.

[48] P. Mayr, $\mathcal{N}=1$ mirror symmetry and open/closed string duality, hep-th/0108229.

[49] P. Mayr, Summing up open string instantons and $\mathcal{N}=1$ string amplitudes, hep-th/0203237.

[50] D.R. Morrison, Through the looking glass, in 'Mirror Symmetry III' eds. D.H. Phong, L. Vinet and S.-T. Yau, International Press, 1999, 263.

[51] T. Oda and H.S. Park, Linear Gale transforms and Gel'fand-KapranovZelevinskij decompositions, Tohoku Math. J. 43 (1991), 375.

[52] H. Ooguri and C. Vafa, Knot invariants and topological strings, Nucl. Phys. B 577 (2000), 419, hep-th/9912123. 
[53] H. Ooguri and C. Vafa, Worldsheet derivation of a large $N$ duality, hep-th/0205297.

[54] P. Ramadevi and T. Sarkar, On link invariants and topological string amplitudes, Nucl. Phys. B 600 (2001), 487, hep-th/0009188.

[55] A. Strominger, Black hole condensation and duality in string theory, Nucl. Phys. Proc. Suppl. 46 (1996), 204, hep-th/9510207.

[56] C. Vafa, Extending mirror conjecture to Calabi-Yau with bundles, hep-th/9804131.

[57] C. Vafa, Superstrings and topological strings at large N, J. Math. Phys. 42 (2001), 2798, hep-th/0008142.

[58] E. Witten, Chern-Simons gauge theory as a string theory, in 'The Floer Memorial Volume' eds. H. Hofer et al, Birkhäuser, 1995, 637, hep-th/9207094. 